Pacific Journal of Mathematics

FORMAL REDUCTION THEORY OF MEROMORPHIC
DIFFERENTIAL EQUATIONS: A GROUP THEORETIC VIEW 


\title{
FORMAL REDUCTION THEORY \\ OF MEROMORPHIC DIFFERENTIAL EQUATIONS: A GROUP THEORETIC VIEW
}

\author{
DONALD G. BABbitT AND V. S. VARAdARAJAN
}

One of the main goals of this paper is to develop an algorithm for reducing the first order (singular) system of differential equations:

$$
\frac{d f}{d z}=A(z) f
$$

to a Turrittin-Levelt canonical form. Here $A(z)=z^{r} A_{r}+z^{r+1} A_{r+1}$ $+\cdots, r<-1$ and $A_{r+m} \in \mathfrak{g l}(n ; \mathbf{C}) m \geq 0$. The reduction of $(\dagger)$ to a canonical form is implemented by the natural gauge adjoint action of $\mathrm{GL}(n ; \overline{\mathscr{F}})$ where $\overline{\mathscr{F}}$ is the algebraic closure of the field of formal Laurent series about 0 with at most a finite pole at 0 . For example, it is shown that the irregular part of the canonical form ( $\dagger$ ) is determined by $A_{r+m}$, $0 \leq m<n(|r|-1)$. The proofs utilize group theoretic techniques as well as the method of Galois descent. In particular almost all of the results generalize to the case where $\operatorname{GL}(n)$ and $g \mathfrak{l}(n)$ are replaced by an arbitrary affine algebraic group $G$ over $\mathrm{C}$ and its Lie algebra $g$.

\section{CONTENTS}

0 . Introduction and summary

1. Notation. Generalities

2. Orbits in complex reductive Lie algebras

The Classical Connections

3. Reduction theory in the regular case

4. Transformations of connections with nilpotent leading coefficients

5. The principal level

6. Reduction theory over $\overline{\mathscr{y}}$

7. Reduction theory over $\mathscr{F}$

Connections with an Affine Algebraic Structure Group

8. Regular connections and their monodromy

9. Reduction theory for arbitrary connections

References

0. Introduction and summary.

0.1 . This paper presents a formal classification of meromorphic linear ordinary differential equations from a group theoretic point of view. Let 
the equations be

$$
\frac{d}{d z} f=A(z) f, \quad f=\left(f_{i}\right)_{1 \leq i \leq n}, \quad A=\left(a_{i j}\right)_{1 \leq i, j \leq n}
$$

where the $n \times n$ matrix $A$ is analytic around the origin and has at most a pole of finite order there. Changing $f$ to $g=x f$ where $x$ is an invertible $n \times n$ matrix, meromorphic in $z$ (or at least in $z^{1 / m}$ for some integer $m \geq 1)$ changes $(0.1)$ to

$$
\frac{d}{d z} g=B(z) g
$$

where

$$
B=x[A]=x A x^{-1}+\dot{x} x^{-1} \quad\left(\dot{x}=\frac{d}{d z} x\right) .
$$

If we introduce the field $\mathscr{F}$ of all formal Laurent series in $z$ and its algebraic closure $\overline{\mathscr{F}}$, it is easy to check from the definition $(0.3)$ that $x, A \mapsto x[A]$ defines an action of $\operatorname{GL}(n, \overline{\mathscr{F}})$ on $g l(n, \overline{\mathscr{F}})$. The proposed treatment of the systems $(0.1)$ is based on a "parametrization" of the orbits for this action.

The determination of canonical forms for $(0.1)$ is quite well known and goes back to Turrittin [26], Levelt [16], Jurkat [11], and more recently, to Robba [21a] (cf. [1]). Our approach, which reduces the problem to the study of the adjoint action of $\operatorname{GL}(n, \mathbf{C})$ on $\mathfrak{g l}(n, \mathbf{C})$, is new. It is moreover completely finitistic and leads to algorithms and sharp bounds on the number of coefficients of the Laurent expansion of $A \in \mathfrak{g l}(n, \overline{\mathcal{F}})$ that are needed to predict the canonical reduction of $A$. The reduction theory over $\mathcal{F}$ itself is treated by Galois descent.

The transformation law $(0.3)$ is that of a connection so that the theory of the systems (0.1) is really the theory of meromorphic connections on vector bundles. When properly formulated (taking into account tensor products) the classical theory would also yield the generalization where $\operatorname{GL}(n, \overline{\mathscr{F}})$ and $\mathfrak{g} \mathfrak{l}(n, \overline{\mathscr{F}})$ get replaced by $G(\overline{\mathscr{F}})$ and $\mathfrak{g}(\overline{\mathscr{F}})$ respectively for any algebraic subgroup $G$ of $\operatorname{GL}(n, \mathbf{C})$ with Lie algebra $g .{ }^{\prime}$ Our methods, operating as they do directly in the context of algebraic subgroups of $\mathrm{GL}(n)$, seem to have interest of their own. For treatments of various aspects of $(0.1)$ from the point of view of connections on vector bundles the reader should consult [1], [7], [13], [18].

\footnotetext{
${ }^{1}$ We owe this remark to Professor P. Deligne.
} 
Finally it is clear that these questions may be formulated in a much more general context where the field $\mathscr{F}$ is replaced by a local field $K$ whose residue field $k$ has characteristic 0 . It is not particularly difficult to do this at least when $k$ is algebraically closed. It is also easy to formulate our main results in a way that does not depend on the choice of a uniformisant for $K$ (see [18], [1]).

Acknowledgement. One of us (V) would like to thank Professor P. Deligne for his many illuminating comments and for his generosity in making himself available. He would also like to thank the authorities of the Mathematics Institute of the State University of Utrecht in Utrecht, Holland, for their hospitality during the winter of 1981 when a part of this work was carried out, as well as his many friends there who made this stay so memorable. The first author would like to thank D. Kastler, J. M. Combes and M. Flato for their warmth and hospitality during his stay in France during the spring of 1981. We would also like to thank Debbie Remetch for the patience and skill with which she prepared this manuscript.

0.2 . There are three parts to this paper. The first part consists of $\S \S 1$ and 2 . $\$ 1$ contains the terminology and notation that will be used in the paper as well as some useful general remarks. $\$ 2$ contains a discussion of the orbits under the action of a complex reductive group on its Lie algebra. The results discussed here are basic to everything that we do later on. We mention in particular the results in $\$ 2.2$ which construct, for a given nilpotent orbit, a transversal having the crucial property that any other nilpotent orbit it meets must have strictly higher dimension; this increase in dimension lies at the heart of all the inductive arguments and algorithms of this paper.

In the second part $(\S \S 3-7)$ we carry out the classification of the orbits in $\mathfrak{g} l(V(\overline{\mathscr{F}}))$ as well as $\mathfrak{g} l(V(\mathscr{F}))$. The core of our method (for reduction over $\overline{\mathscr{F}})$ is a technique developed in $§ 4.6$; it shows how to transform a given connection $A \in \mathrm{gl}(V(\overline{\mathscr{F}}))$ of order $r<-1$ and nilpotent leading coefficient $A_{r}$, into a connection $A^{\prime}$ of order $r^{\prime} \geq r$, such that, the leading coefficient $A^{\prime}{ }^{\prime}$, of $A^{\prime}$ is either non-nilpotent, or is nilpotent, but belongs to a $G$-orbit of dimension greater than that of the orbit of $A_{r}$. In combination with the well known spectral splitting of a connection (along the spectral subspaces of its leading coefficient) this leads in $\S 6$ to the main results of classical reduction theory, and, in addition, to algorithms for obtaining the reductions. As an illustration of the algorithmic aspects of our techniques we mention the result that the entire irregular part of the 
reduced form of $A$ is determined by the coefficients $A_{r+s}, 0 \leq s<$ $n(|r|-1)(n=\operatorname{dim}(V))$, the estimate being sharp. Earlier results of this kind were restricted to just the term of the highest singularity in the reduced form (cf. [12]). In $\$ 7$ we show how the reduction theory over $\overline{\mathcal{F}}$, combined with elementary techniques of Galois descent, yields the reduction theory over $\mathscr{F}$.

In the third part ( $\S 8-9)$ we show that the methods of $\S \S 4-7$ go over essentially without change to the context where $\mathrm{GL}(V)$ is replaced by any complex affine algebraic group. The main results remain virtually the same.

The material in $\S \S 3-7$ is self-contained and, taking into account $\S \S 1$ and 2, does not require any special background of the reader. The treatment in $\$ \S 8$ and 9 however assumes some familiarity with the theory of algebraic groups, and we have tried to compensate by giving extensive reference to the relevant parts of Borel's book [3].

\section{Notation. Generalities.}

1.1. We begin by introducing our basic notation. As usual $\mathbf{Z}, \mathbf{Q}$, and C will respectively stand for the ring of integers, the field of rational numbers, and the field of complex numbers. Let $z$ be a complex variable and $\mathscr{F}=\mathscr{F}_{z}$ the field of all formal Laurent series

(1.1) $f=\sum_{j \in \mathbf{Z}} f_{j} z^{j}, \quad f_{J}=0$ if $j<0$ and $|j|$ is sufficiently large.

If $f$ in (1.1) is nonzero, its order, ord $(f)$, is the integer $k$ such that $f_{k} \neq 0$ and $f_{J}=0$ for $j<k$; we put $\operatorname{ord}(0)=+\infty$. The ring of elements of order $\geq 0$ is denoted by 0 . The Laurent series (1.1) is said to be convergent if for some $\tau \neq 0$ in $\mathbf{C}$ the series $\Sigma\left|f_{j}\right| \mid \tau \psi^{j}$ is convergent. The convergent elements form a subfield of $\mathscr{F}$, denoted by $\mathscr{F}_{\text {cgt }}$; we put $\theta_{\text {cgt }}=\theta \cap \mathscr{F}_{\text {cgt }}$.

Let $\overline{\mathscr{F}}$ be the algebraic closure of $\mathscr{F}$. Then, by the classical theorem of Puiseux, $\overline{\mathscr{F}}=\bigcup_{b \geq 1} \mathscr{F}_{b}$ where $\mathscr{F}_{b}$ is the Galois extension $\mathscr{F}\left(z^{1 / b}\right)$. Fixing a choice of $z^{1 / b}=\zeta$ gives rise to an isomorphism, called the $\zeta$-lifting, of $\mathscr{F}_{b}$ with $\widetilde{F}_{\zeta}$; the $\zeta$-lifting of $f=\sum a_{j}\left(z^{1 / b}\right)^{\jmath}$ is $\sum a_{j} \zeta^{j}$ and will be denoted by $\hat{f}$. The pre-images of $\mathscr{F}_{\zeta, \text { cgt }}, \vartheta_{\zeta}$, in $\mathscr{F}_{b}$ will be denoted respectively by $\mathscr{F}_{b, \text { cgt }}, \theta_{b}$. If $f \in \mathscr{F}_{b}$ we write ord $(f)$ for $(1 / b) \operatorname{ord}(\hat{f})$. These are independent of the choice of $z^{1 / b}$. Let $\mu_{b}$ be the multiplicative group of $b$ th roots of unity. We have $\mu_{b} \simeq \operatorname{Gal}\left(\mathscr{F}_{b} / \mathscr{F}\right)$; the element $\gamma \in \mu_{b}$ acts on $\mathscr{F}_{b}$ via

$$
f=\sum_{j} a_{j}\left(z^{1 / b}\right)^{J} \mapsto \gamma \cdot f=f^{\gamma}=\sum_{j} a_{j} \gamma^{-J}\left(z^{1 / b}\right)^{J}
$$


This is also independent of the choice of $z^{1 / b}$. The order functions on the $\mathscr{F}_{b}$ are mutually compatible. We write $\bar{\theta}$ for $\cup_{b \geq 1} \theta_{b}$. The field $\overline{\mathscr{F}}_{\text {cgt }}=$ $\cup_{b \geq 1} \mathscr{F}_{b, \mathrm{cgt}}$ is the algebraic closure of $\mathscr{F}_{\text {cgt }}$. Finally, as is well known, we may regard the $\mathscr{F}_{b}$ as topological fields; as a fundamental system of neighborhoods of 0 in this topology, the so-called adic topology, we take the ideals $\theta_{b, m}=\Theta_{b} z^{m / b}(m \geq 0)$.

The derivation $d / d z$ of $\mathscr{F}$ extends uniquely to a derivation of $\overline{\mathscr{F}}$, also denoted by $d / d z$. For $f \in \overline{\mathscr{F}}$ we often write $\dot{f}$ for $d f / d z$. If $u \in \overline{\mathscr{F}}$ is not a constant, $d / d u$ is defined as the derivation $(\dot{u})^{-1} d / d z$ of $\overline{\mathscr{F}}$. If $b \geq 1, d / d \zeta$ and $d / d\left(z^{1 / b}\right)$ correspond under the $\zeta$-lifting isomorphism:

$$
\frac{d}{d \zeta} \hat{x}=b \zeta^{b-1} \hat{x}, \quad x \in \overline{\mathscr{F}},
$$

here we are extending the $\zeta$-lifting to some isomorphism $x \mapsto \hat{x}$ of $\overline{\mathcal{F}}$ with $\overline{\mathscr{F}}_{\xi}$. If $\gamma \in \operatorname{Gal}(\overline{\mathscr{F}} / \mathscr{F})$ and $u \in \overline{\mathscr{F}}(\dot{u} \neq 0)$, we have $(\dot{u})^{\gamma}=\left(u^{\gamma}\right)^{\dot{*}}$; and $\gamma(d / d u) \gamma^{-1}=d / d u^{\gamma}$.

1.2. Let $V$ be a complex vector space of finite dimension $n$ over $\mathbf{C}$. For any commutative $\mathbf{C}$-algebra $R$ we put

$V(R)=V \otimes_{\mathrm{C}} R, \quad \mathfrak{g l}(V(R))=\mathfrak{g l}(V) \otimes_{\mathrm{C}} R, \quad \mathrm{GL}(V(R))=\operatorname{Aut}_{R} V(R)$.

The cases $R=\mathscr{F}, \overline{\mathscr{F}}, \vartheta$, etc., will be important to us.

The notions of order, convergence, and $\zeta$-lifting extend in the obvious way to $V(\overline{\mathscr{F}})$. Moreover we obtain an isomorphism

$$
\operatorname{GL}\left(V\left(\mathscr{F}_{b}\right)\right) \stackrel{\sim}{\rightarrow} \mathrm{GL}\left(V\left(\mathscr{F}_{\zeta}\right)\right) \quad(x \mapsto \hat{x})
$$

which is the restriction to $\operatorname{GL}\left(V\left(\mathscr{F}_{b}\right)\right.$ ) of the $\zeta$-lifting isomorphism (of associative algebras) $\operatorname{End}\left(V\left(\mathscr{F}_{b}\right)\right) \stackrel{\sim}{\rightarrow} \operatorname{End}\left(V\left(\mathscr{F}_{\zeta}\right)\right)$.

For $R=\theta, \operatorname{GL}(V(\theta))$ is the group of $x \in \mathrm{GL}(V(\mathscr{F}))$ of the form $T_{0}+z T_{1}+\cdots$ where $T_{0} \in \mathrm{GL}(V)$; for $m \geq 1$, GL $(V(\theta))_{m}$ is the subgroup of $x \in \operatorname{GL}(V(\theta))$ with $x \equiv 1 \bmod \left(z^{m}\right)$. For instance, if $T \in \mathfrak{g l}(V)$, $\exp \left(z^{m} T\right) \in \mathrm{GL}(V(\theta))_{m}$.

We write $d / d u(u \in \overline{\mathscr{F}}, \dot{u} \neq 0)$ for the unique $\mathbf{C}$-linear map of $V(\overline{\mathscr{F}})$ such that

$$
\frac{d}{d u}(f w)=\left(\frac{d}{d u} f\right) w+f \frac{d}{d u} w \quad(f \in \overline{\mathscr{F}}, w \in V(\overline{\mathscr{F}})) .
$$


For $\sigma \in \operatorname{Gal}(\overline{\mathscr{F}} / \mathscr{F})$, we write $w \mapsto w^{\sigma}$ for the unique C-linear automorphism of $V(\overline{\mathscr{F}})$ such that

$$
(f v)^{\sigma}=f^{\sigma} v \quad(f \in \overline{\mathscr{F}}, v \in V) .
$$

We have the obvious isomorphism

$$
\mathrm{GL}(V(\theta)) / \mathrm{GL}(V(\theta))_{1} \stackrel{\sim}{\rightarrow} \mathrm{GL}(V) .
$$

If $m$ is any integer $\geq 1$ the map

$$
1+z^{m} T_{m}+z^{m+1} T_{m+1}+\cdots \mapsto T_{m}
$$

gives rise to the isomorphism ( + denotes additive group)

$$
\mathrm{GL}(V(\theta))_{m} / \mathrm{GL}(V(\theta))_{m+1} \simeq \mathfrak{g l}(V)_{+} \quad(m \geq 1) .
$$

The formulae (1.7) are further clarified if we use infinite product representations for elements of $\mathrm{GL}(V(\theta))_{1}$. It is easy to check that $\mathrm{GL}(V(\theta))_{1}$ is precisely the set of all infinite products

(1.7c) $\quad x=\prod_{k=1}^{\infty}\left(1+z^{k} T_{k}\right) \underset{\mathrm{dfn}}{=} \lim _{m \rightarrow \infty}\left(1+z^{m} T_{m}\right) \cdots\left(1+z T_{1}\right)$

the elements $T_{m}$ being uniquely determined, given $x$, by

$$
x\left(\left(1+z^{m} T_{m}\right) \cdots\left(1+z T_{1}\right)\right)^{-1} \equiv 1 \quad\left(\bmod z^{m+1}\right) \quad(m \geq 0) .
$$

The subgroup $\operatorname{GL}\left(V\left(\theta_{b}\right)\right)$ of $\operatorname{GL}\left(V\left(\mathscr{F}_{b}\right)\right)$ gives rise to its "polar decomposition." Let $V=\mathbf{C}^{n}$, so that $\operatorname{GL}\left(V\left(\mathscr{F}_{b}\right)\right)=\operatorname{GL}\left(n, \mathscr{F}_{b}\right)$ and $\operatorname{GL}\left(V\left(\theta_{b}\right)\right)=\operatorname{GL}\left(n, \theta_{b}\right)$. Let a choice of $z^{1 / b}$ be fixed, let $z^{m / b}$ $={ }_{\mathrm{dfn}}\left(z^{1 / b}\right)^{m}(m \in \mathbf{Z})$ and let

$$
\mathscr{Q}_{+, b}=\left\{\operatorname{diag}\left(z^{r_{1}}, \ldots, z^{r_{n}}\right) \mid r_{J} \in(1 / b) \mathbf{Z}, r_{1} \leq r_{2} \leq \cdots \leq r_{n}\right\} .
$$

Then it follows from the theory of elementary divisors ([4], Th. 1, p. 82) that

$$
\operatorname{GL}\left(n, \mathscr{F}_{b}\right)=\operatorname{GL}\left(n, \vartheta_{b}\right) \mathscr{Q}_{+, b} \operatorname{GL}\left(n, \theta_{b}\right)
$$

moreover, given $x \in \mathrm{GL}\left(n, \mathscr{F}_{b}\right)$, its component in $\mathscr{Q}_{+, b}$ with respect to the decomposition (1.9a) is uniquely determined. In particular,

$$
\operatorname{GL}\left(n, \vartheta_{b}\right) \backslash \mathrm{GL}\left(n, \mathscr{F}_{b}\right) / \mathrm{GL}\left(n, \mathcal{\theta}_{b}\right) \stackrel{\sim}{\rightarrow} \mathscr{D}_{+, b} .
$$

1.3. We shall refer (cf. the introduction) to elements of $\mathfrak{g} l(V(\overline{\mathscr{F}}))$ as connections. GL $(V(\overline{\mathscr{F}}))$ acts on $\operatorname{gl}(V(\overline{\mathscr{F}}))$ by

$$
x[A]=x A x^{-1}+\dot{x} x^{-1} \quad(x \in \operatorname{GL}(V(\overline{\mathscr{F}})), A \in \mathfrak{g} \mathfrak{l}(V(\overline{\mathscr{F}}))) .
$$


Note the identity

$$
x[A+B]=x A x^{-1}+x[B]=x[A]+x B x^{-1}
$$

as well as the following formula relating the actions of $\operatorname{GL}(V(\overline{\mathscr{F}}))$ and $\operatorname{Gal}(\overline{\mathscr{F}} / \mathscr{F})$ :

$$
\begin{aligned}
& (x[A])^{\sigma}=x^{\sigma}\left(\left[A^{\sigma}\right]\right) \\
& \quad(x \in \mathrm{GL}(V(\overline{\mathscr{F}}))), \sigma \in \mathrm{Gal}(\overline{\mathscr{F}} / \mathscr{F}), A \in \mathfrak{g} \mathfrak{l}(V(\overline{\mathscr{F}})) .
\end{aligned}
$$

In particular, if $A$ is in $\mathfrak{g} \mathfrak{l}(V(\mathscr{F}))$ its equivalence class under $\operatorname{GL}(V(\overline{\mathscr{F}}))$ (resp. $\operatorname{GL}\left(V\left(\mathscr{F}_{b}\right)\right)$ ) is fixed by $\operatorname{Gal}(\overline{\mathscr{F}} / \mathscr{F})$ (resp. $\operatorname{Gal}\left(\mathscr{F}_{b} / \mathscr{F}\right)$ ). In $\S 7.4$ we shall see that the converse statement is true and forms the point of departure for a complete description of the $\operatorname{GL}(V(\mathscr{F}))$-equivalence classes of elements of $\mathfrak{g l}(V(\mathscr{F}))$ that are equivalent to $A$ under $\operatorname{GL}\left(V\left(\mathscr{F}_{b}\right)\right)$.

Given $b \geq 1$ and a fixed choice of $z^{1 / b}$ the $\zeta$-lifting map $\mathfrak{g l}\left(V\left(\mathscr{F}_{b}\right)\right) \rightarrow$ $\mathfrak{g} \mathfrak{l}\left(V\left(\mathscr{F}_{\xi}\right)\right)$ will not commute with the actions of $\operatorname{GL}\left(V\left(\mathscr{F}_{b}\right)\right)$ and $\operatorname{GL}\left(V\left(\mathscr{F}_{\zeta}\right)\right)$. We shall therefore modify it. The modified map, applicable only to connections, will be denoted by $\sim$. Given

$$
A=\sum_{j \in \mathbf{Z}} A_{j / b} z^{j / b} \in \mathfrak{g l}\left(V\left(\mathscr{F}_{b}\right)\right)
$$

we define

$$
\tilde{A}=b \zeta^{b-1} \sum_{j \in \mathbf{Z}} A_{J / b} \zeta^{j}=b \zeta^{b-1} \hat{A} .
$$

With this definition it is easy to check that

$$
\hat{x}[\tilde{A}]=\widetilde{x[A]} \quad\left(x \in \operatorname{GL}\left(V\left(\mathscr{F}_{b}\right)\right), A \in \mathfrak{g} \mathfrak{l}\left(V\left(\mathscr{F}_{b}\right)\right)\right) .
$$

For the Galois actions we have the formula

$$
\widetilde{\gamma \cdot A}=\gamma^{-1} \tilde{A}^{\gamma} \quad\left(\tilde{A}^{\gamma}(\zeta)=\tilde{A}\left(\gamma^{-1} \zeta\right), \gamma \in \mu_{b}\right) \text {. }
$$

We also note from (1.14) the obvious

$$
\operatorname{ord}(\tilde{A})=b \operatorname{ord}(A)+b-1 .
$$

1.4. We wish to make a few remarks concerning the relation between linear ordinary differential equations and connections; for a detailed treatment in complete generality see Deligne ([7], pp. 23-29). If we are given an ordinary differential equation

$$
\left(\frac{d}{d z^{n}}+a_{1} \frac{d^{n-1}}{d z^{n-1}}+\cdots+a_{n-1} \frac{d}{d z}+a_{n}\right) y=0
$$


we convert it into the system

$$
\left(\frac{d}{d z}-A\right) f=0, \quad f=\left(\begin{array}{c}
y \\
y^{\prime} \\
\vdots \\
y^{(n-1)}
\end{array}\right)
$$

where

$$
A=\left(\begin{array}{cccccc}
0 & 1 & & & & \\
& 0 & 1 & & 0 & \\
& & 0 & \ddots & & \\
0 & & & \ddots & 1 & \\
-a_{n}, & -a_{n-1} & , \ldots, & & -a_{2}, & -a_{1}
\end{array}\right)
$$

The converse is also true; any connection $\in \mathfrak{g l}(n, \overline{\mathcal{F}})$ is equivalent to one of the form (1.18), so that it arises from a scalar $n$th order ordinary differential equation (cf. [7]; also [6]). Since our primary focus is on connections and their transformation properties, we do not use this result in this paper.

1.5. We now wish to extend the discussion of the preceding paragraphs to the case where $\mathrm{GL}(V)$ is replaced by an arbitrary connected affine algebraic group over $\mathbf{C}$. We shall generally follow the notation and terminology of Borel ([3], Chapter I). Fix such a group $\mathbf{G}$, and write, for any $\mathbf{C}$-algebra $R, G(R)$ for the group of $R$-points of $\mathbf{G}$; we write $G=G(\mathbf{C})$. Let $g$ be the Lie algebra of $G$. For any $\mathbf{C}$-algebra $R$ let

$$
\mathfrak{g}(R)=\mathfrak{g} \otimes_{\mathrm{C}} R \text {. }
$$

If $\rho\left(G \rightarrow G^{\prime}\right)$ is a morphism of algebraic groups defined over $\mathbf{C}$, its differential $d \rho$ is a Lie algebra morphism $g \rightarrow \mathfrak{g}^{\prime}$ where $\mathfrak{g}^{\prime}=\operatorname{Lie}\left(G^{\prime}\right)$.

As in the case of GL(V) we have the subgroup $G(\vartheta)$ of $G(\mathscr{F})$ and the "congruence subgroups" $G(\theta)_{m}(m \geq 1)$. For instance, if $G \subset \mathrm{GL}(V)$, $G(\vartheta)_{m}=G(\mathscr{F}) \cap \mathrm{GL}(V(\vartheta))_{m}$. If $T \in \mathfrak{g}, \quad \exp \left(z^{m} T\right) \in G(\vartheta)_{m}$ for any $m \geq 1$. It is easy to show that $G(\theta)_{1}$ is precisely the set of elements of the form

(1.20a) $\quad x=\prod_{k=1}^{\infty} \exp \left(z^{k} T_{k}\right) \underset{\mathrm{dfn}}{=} \lim _{m \rightarrow \infty} \exp \left(z^{m} T_{m}\right) \cdots \exp \left(z T_{1}\right)$

where $T_{J}$ are uniquely defined by

(1.20b) $x\left(\exp \left(z^{m} T_{m}\right) \cdots \exp \left(z T_{1}\right)\right)^{-1} \in G(\theta)_{m+1} \quad(m \geq 0)$. 
The $\zeta$-lifting isomorphism leads, as in the case of $\mathrm{GL}(V)$, to the maps $x \mapsto \hat{x}$ (of $G\left(\mathscr{F}_{b}\right)$ with $G\left(\mathscr{F}_{\xi}\right)$ ) and $A \mapsto \tilde{A}$ (of $\mathrm{g}\left(\mathscr{F}_{b}\right)$ with $\mathfrak{g}\left(\mathscr{F}_{\xi}\right)$ ). One can also show that $G\left(\mathscr{F}_{b, \mathrm{cgt}}\right)$ is dense (adic topology) in $G\left(\mathscr{F}_{b}\right)$. This is easy to verify directly if $T$ is a torus or unipotent; for semisimple $G$, it follows from the Iwasawa decomposition of $G(\mathscr{F})([25]$, p. 114); for general $G$, from its Levi decomposition.

If $H$ is an endomorphism of a finite dimensional vector space $V$ over $\mathbf{C}$ which is semisimple and has only integral eigenvalues, it makes sense to speak of the element $z^{H}$ in $\operatorname{GL}(V(\mathscr{F}))$; if $\lambda \in \mathbf{Z}$ and $V_{\lambda}$ is the eigenspace on which $H$ acts as $\lambda \cdot$ id, $z^{H}$ acts as $z^{\lambda}$. id on $V_{\lambda}$. Clearly $z^{H} \in \operatorname{GL}\left(V\left(\widetilde{F}_{\text {cgt }}\right)\right)$ and commutes with any endomorphism of $V$ that commutes with $H$. Suppose $b$ is an integer $\geq 1$ and we have fixed a choice of $z^{1 / b}$. We define, for any $\lambda=\mu / b \in(1 / b) \mathbf{Z}, z^{\lambda}$ as $\left(z^{1 / b}\right)^{\mu}$. If $H$ above is assumed to have all its eigenvalues in $(1 / b) \mathbf{Z}$, we can define $z^{H}$ exactly as before; it lies in $\operatorname{GL}\left(V\left(\mathscr{F}_{b, c \mathrm{t}}\right)\right)$ and commutes with all elements in the centralizer of $H$. To extend these definitions to the case of a complex affine algebraic group $G$ with Lie algebra $g$, we use affine imbeddings $G \hookrightarrow \mathrm{GL}(V)$. The notion of an integral element $X$ of $g$ makes sense: $X$ is integral if and only if in some affine imbedding it has only integral eigenvalues; then $d \rho(X)$ has only integral eigenvalues for any $\mathbf{C}$-morphism $\rho(G \rightarrow \mathrm{GL}(V))$. It is now easy to show that for any integer $b \geq 1$ and a fixed choice of $z^{1 / b}$, we have a map

$$
H \mapsto z^{H}
$$

from the set of semisimple $H \in \mathfrak{g}$ for which $b H$ is integral, to $G\left(\mathscr{F}_{b, \mathrm{cgt}}\right)$. Given $H, z^{H}$ is characterized by

$$
\rho\left(z^{H}\right)=z^{d \rho(H)} \quad(\rho=\text { any } \mathbf{C} \text {-morphism } G \rightarrow \mathrm{GL}(V)) .
$$

If $H, H^{\prime}$ commute,

$$
z^{H+H^{\prime}}=z^{H} z^{H^{\prime}}
$$

1.6. Given $x \in G\left(\mathscr{F}_{b}\right)$ one can show the existence of a unique element $\delta_{G}(x) \in \mathfrak{g}\left(\widetilde{F}_{b}\right)$ such that for any C-morphism $\rho(G \rightarrow \mathrm{GL}(V))$,

$$
d \rho\left(\delta_{G}(x)\right)=\rho(x)^{\bullet} \rho(x)^{-1} .
$$

In fact it is easy to see this for $x \in G\left(\mathscr{F}_{b, \mathrm{cgt}}\right)$; for general $x$ one uses a density argument. If $\rho\left(G \rightarrow G^{\prime}\right)$ is a morphism of algebraic groups,

$$
d \rho\left(\delta_{G}(x)\right)=\delta_{G^{\prime}}(\rho(x)) \quad(x \in G(\overline{\mathscr{F}})) .
$$


Using this we can define an action of $G(\overline{\mathscr{F}})$ on $g(\overline{\mathscr{F}})$ by

$$
x[A]=\operatorname{Ad}(x)(A)+\delta_{G}(x) .
$$

In the presence of the transformation law (1.26) we shall refer to elements of $g(\overline{\mathscr{F}})$ as connections.

The definition (1.26) reduces to (1.10) for $G=\mathrm{GL}(V)$ and is related to it by (1.24). The relation (1.24) allows one to extend almost all the remarks made earlier to the present context. For later use we put down the following consequence of (1.25):

$$
d \rho(x[A])=\rho(x)[d \rho(A)] .
$$

1.7. If $x \in \mathrm{GL}(V(\bar{\theta}))$ and $A \in \mathfrak{g l}(V(\overline{\mathscr{F}}))$, $\operatorname{ord}(x[A])=\operatorname{ord}(A)$. For $x \notin \mathrm{GL}(V(\bar{\theta}))$ this is no longer true in general. For $x \in \mathrm{GL}(V(\mathscr{F}))$ we define $\operatorname{lag}(x)$ as the smallest of the integers $m \geq 0$ such that $A \equiv 0$ $\left(\bmod z^{m}\right)$ implies $x A x^{-1} \in \mathfrak{g l}(V(\theta))(A \in \mathfrak{g} \mathfrak{l}(V(\mathscr{F})))$. If $\operatorname{lag}(x) \leq m$, then (1.28) $A, B \in \mathfrak{g} \mathfrak{l}(V(\mathscr{F})), \quad A \equiv B\left(\bmod z^{r}\right) \Rightarrow x[A] \equiv x[B]\left(\bmod z^{r-m}\right)$. For $b \geq 1$ we use the $\zeta$-lifting to define (independently of the choice of $\left.z^{1 / b}\right)$

$$
\operatorname{lag}(x)=(1 / b) \operatorname{lag}(\hat{x}) \quad\left(x \in \mathrm{GL}\left(V\left(\mathscr{F}_{b}\right)\right)\right) .
$$

The definitions (1.29) are compatible when $b$ varies. The following is a routine consequence of the definitions. In view of (1.9), (1.30) leads to the effective computability of $\operatorname{lag}(x)$ for all $x$.

Proposition 1. We have the following.

(i) $\operatorname{lag}(x)=0$ for $x \in \mathrm{GL}\left(V\left(\theta_{b}\right)\right) \cdot Z_{b}$ where $Z_{b}$ is the group of elements of the form $z^{k} \cdot 1, k \in(1 / b) \mathbf{Z}$.

(ii) $\operatorname{lag}(x y) \leq \operatorname{lag}(x)+\operatorname{lag}(y)$ for all $x, y \in \mathrm{GL}\left(V\left(\mathscr{F}_{b}\right)\right)$.

(iii) $\operatorname{lag}\left(y x y^{\prime}\right)=\operatorname{lag}(x)$ if $y, y^{\prime} \in \mathrm{GL}\left(V\left(\vartheta_{b}\right)\right) \cdot Z_{b}, x \in \mathrm{GL}\left(V\left(\mathscr{F}_{b}\right)\right)$.

(iv) If $x=z^{H}$ where $H \in \mathrm{gl}(V)$ is semi-simple and has eigenvalues $k_{1}, \ldots, k_{n} \in(1 / b) \mathbf{Z}$,

$$
\operatorname{lag}(x)=\max _{1 \leq i, j \leq n}\left|k_{i}-k_{j}\right| .
$$

(v) $\operatorname{lag}(x)=\operatorname{lag}\left(x^{-1}\right), x \in \mathrm{GL}\left(V\left(\mathscr{F}_{b}\right)\right)$.

Let $\bar{\theta}^{\times}$be the group of units of $\bar{\theta}$. We define

$$
{ }^{\circ} \mathrm{GL}(V(\overline{\mathscr{F}}))=\left\{x \in \mathrm{GL}(V(\overline{\mathscr{F}})) \mid \operatorname{det}(x) \in \overline{\mathcal{O}}^{\times}\right\} .
$$


If $V=\mathrm{C}^{n}$ we write ${ }^{\circ} \mathrm{GL}(n, \overline{\mathscr{F}})$ for ${ }^{\circ} \mathrm{GL}(V(\overline{\mathscr{F}}))$. It is clear that ${ }^{\circ} \mathrm{GL}(V(\overline{\mathscr{F}}))$ is a subgroup of $\operatorname{GL}(V(\overline{\mathscr{F}}))$ and that

$$
\mathrm{SL}(V(\overline{\mathscr{F}})) \subset{ }^{\circ} \mathrm{GL}(V(\overline{\mathscr{F}})), \quad \mathrm{GL}(V(\bar{\theta})) \subset{ }^{\circ} \mathrm{GL}(V(\overline{\mathscr{F}})) .
$$

Moreover, if $H$ is semisimple in $\mathfrak{g l}(V)$ with eigenvalues in $\mathbf{Q}$, it is immediate that

$$
z^{H} \in{ }^{\circ} \mathrm{GL}(V(\overline{\mathscr{F}})) \Leftrightarrow \operatorname{tr}(H)=0 .
$$

Proposition 2. Let $V=V_{1} \oplus \cdots \oplus V_{m}, y=y_{1} \oplus \cdots \oplus y_{m}$ where $y_{l} \in$ ${ }^{\circ} \mathrm{GL}\left(V_{i}(\overline{\mathscr{F}})\right)$. Then $y \in{ }^{\circ} \mathrm{GL}(V(\overline{\mathscr{F}}))$ and

$$
\operatorname{lag}(y) \leq \operatorname{lag}\left(y_{1}\right)+\cdots+\operatorname{lag}\left(y_{m}\right) .
$$

Proof. Let $y_{i}^{\prime}=1 \oplus \cdots \oplus y_{i} \oplus 1 \oplus \cdots \oplus 1,1 \leq i \leq m$. Then $y_{i}^{\prime} \in$ ${ }^{\circ} \mathrm{GL}(V(\overline{\mathscr{F}}))$ and $y=y_{1}^{\prime} \cdots y_{m}^{\prime}$. So $y \in{ }^{\circ} \mathrm{GL}(V(\overline{\mathscr{F}}))$ and $\operatorname{lag}(y) \leq \operatorname{lag}\left(y_{1}^{\prime}\right)$ $+\cdots+\operatorname{lag}\left(y_{m}^{\prime}\right)$. It is thus sufficient to prove that $\operatorname{lag}\left(y_{i}^{\prime}\right)=\operatorname{lag}\left(y_{i}\right)$ for all $i$. We use the polar decomposition in $\operatorname{GL}\left(V_{l}(\overline{\mathscr{F}})\right)$ to come down to the situation where (with respect to suitable bases for $V_{1}, \ldots, V_{m}$ )

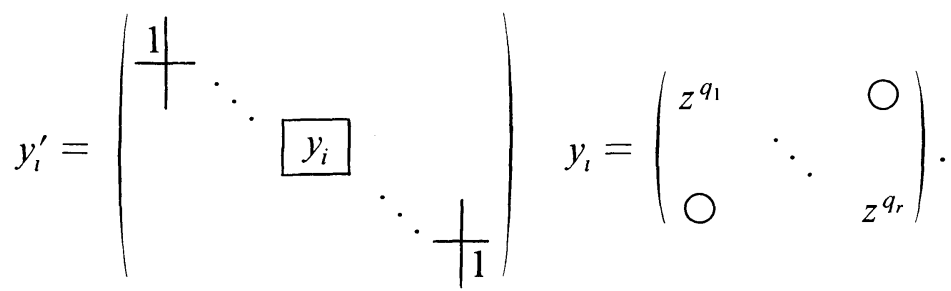

The result is now immediate from (1.30) since $\min _{i} q_{i} \leq 0 \leq \max _{i} q_{i}$.

\section{Orbits in complex reductive Lie algebras.}

2.1. The reduction theory of connections in $g(\overline{\mathscr{F}}), \mathfrak{g}$ being the Lie algebra of a complex reductive group $G$, depends in a fundamental way on the orbit structure of the adjoint representation of $G$. If $G=\operatorname{GL}(n, \mathbf{C})$, the orbits in $\mathfrak{g}=\mathfrak{g l}(n, \mathbf{C})$ are just the similarity classes of matrices. However, the orbit theory for general $G$ may not be familiar to the nonspecialist; and so we devote this section to a brief review of the structure and classification of the orbits of the action of $G$ on $g$. As the basic references we suggest [14] [15] and [24] (cf. also [28], [29], [10]).

Our main results here are in \$2.2. Propositions 2.2.1 and 2.2.2 construct, for any nilpotent $Y \neq 0$ in $\mathrm{g}$, an affine subspace of $\mathrm{g}$ through $Y$, meeting the orbit of $Y$ only at $Y$ and having the property that any other nilpotent orbit that it meets is necessarily of dimension strictly greater 
than the dimension of the orbit of $Y$. This result is basic for everything we do. It controls the inductive mechanisms involved in virtually all aspects of the reduction theory of elements of $g(\overline{\mathscr{F}})$.

The affine transversal mentioned above occurs in other contexts (cf. [10], [28]). While the results of $\$ 2.2$ are contained in [15] when $Y$ is principal, they seem to be new in the general case.

Let $g$ be a reductive Lie algebra over $\mathbf{C}$. Then $[g, g]$ is semisimple and $\mathfrak{g}=[\mathfrak{g}, \mathfrak{g}] \oplus \mathfrak{c}$ where $\mathfrak{c}$ is the center of $\mathfrak{g} . G$ is a complex algebraic group with Lie algebra $\mathfrak{g}$. $G$ operates on $\mathfrak{g}$ via the adjoint representation, and for most of the following remarks one may assume as well that $G$ is the adjoint group.

An element $X \in \mathfrak{g}$ is said to be semisimple if ad $X$ is semisimple; it is called nilpotent if $X \in[\mathfrak{g}, \mathfrak{g}]$ and ad $X$ is nilpotent. For $\mathfrak{g}=\mathfrak{g} \mathfrak{l}(V)$, $[\mathfrak{g}, \mathfrak{g}]=\mathfrak{g} \mathfrak{l}(V)$, and $X \in \mathfrak{g}$ is semisimple or nilpotent in the above sense if and only if it is so in the usual sense as an endomorphism of $V$.

(a) The fundamental fact concerning nilpotents is the theorem of Jacobson-Morozov: let $Y \neq 0$ be a nilpotent in $\mathfrak{g}$ (i.e. $[\mathfrak{g}, \mathfrak{g}]$ ); then we can find $H, X \in[\mathfrak{g}, \mathfrak{g}]$ such that $H, X$ and $Y$ span an $\mathfrak{g l}(2, \mathbf{C})$ with standard commutation rules, i.e.,

$$
[H, X]=2 X, \quad[H, Y]=-2 Y, \quad[X, Y]=H .
$$

Given $Y, H$ is unique upto conjugacy by $G_{Y}$, the centralizer of $Y$ in $G$; given $Y$ and $H, X$ is unique (cf. Kostant [14], 980-988). In particular, the $\mathfrak{g} \backslash(2, \mathbf{C})$ 's containing $Y$ are all conjugate via $G_{Y}$. We note that the commutation rules $(2.1)$ are the same as those satisfied in $\mathfrak{s} l(2, \mathbf{C})$ by

$$
H=\left(\begin{array}{cc}
1 & 0 \\
0 & -1
\end{array}\right), \quad X=\left(\begin{array}{ll}
0 & 1 \\
0 & 0
\end{array}\right), \quad Y=\left(\begin{array}{ll}
0 & 0 \\
1 & 0
\end{array}\right)
$$

$\{H, X, Y\}$ is called a standard triple; $H$ is semisimple, and $X$ is nilpotent. The roles of the elements $X$ and $Y$ can be interchanged in the sense that there is an element $g \in G$ such that $Y^{g}=X, X^{g}=Y, H^{g}=-H$ where $Z^{g}$ is an often used abbreviation for $\operatorname{Ad}(g)(Z)$.

(b) The usefulness of the Jacobson-Morozov theorem of course lies in the fact that it allows us to split $g$ with respect to the adjoint action of the $\mathfrak{g} \mathfrak{I}(2, \mathrm{C})$ spanned by $H, X$ and $Y$. Let

$$
\mathfrak{a}=\mathbf{C} \cdot H+\mathbf{C} \cdot X+\mathbf{C} \cdot Y .
$$

If $W$ is any finite dimensional irreducible $\mathfrak{a}$-module, $H$ acts semisimply on $W$ with eigenvalues $\lambda, \lambda-2, \ldots,-\lambda$ for some integer $\lambda \geq 0$, all eigenvalues being simple. $W$ is said to be of highest weight $\lambda$. In particular, $\operatorname{dim}(W)=\lambda+1$, and if $W_{\mu}$ is the eigenspace for the eigenvalue $\mu$ of $H$, 
we have

$$
\begin{array}{rlrl}
W_{\lambda} & =\{w \in W \mid X w=0\}, & W_{-\lambda}=\{w \in W \mid Y w=0\} \\
\operatorname{range}(Y) & =W_{\lambda-2} \oplus \cdots \oplus W_{-\lambda}, & & \operatorname{range}(X)=W_{\lambda} \oplus \cdots \oplus W_{-\lambda+2} .
\end{array}
$$

Consequently, $W$ is the direct sum of $\operatorname{range}(Y)$ and the null space of $X$. This is then true for direct sums of irreducible modules also. Thus, applying it to $g$ regarded as an $a$-module, we get

$$
\mathrm{g}=\mathrm{g}_{X} \oplus[Y, \mathrm{~g}] \quad\left(\mathrm{g}_{X}=\text { centralizer of } X \text { in } \mathrm{g}\right) .
$$

For convenience we shall refer to $X$ as a nilpotent opposite to $Y$.

Let $U$ be an a-module of finite dimension and let $U_{X}$ be the null space of $X$. It is then $H$-stable and hence we can select a basis $u_{\imath}(1 \leq i \leq q)$ of $U_{X}$ consisting of eigenvectors for $H$. If $H u_{i}=\lambda_{i} u_{i}$, the $\lambda_{l}$ will be integers $\geq 0, u_{l}$ generates an irreducible a-module $U_{l}$ of highest weight $\lambda_{l}$, and $U=U_{1} \oplus \cdots \oplus U_{q}$. In particular, taking $U=\mathfrak{g}$, we see that $\mathfrak{g}_{X}$ is stable under ad $H$; and that if $Z_{i}(1 \leq i \leq q)$ is a basis of $\mathfrak{g}_{X}$ of eigenvectors for ad $H$, we have

$$
\mathfrak{g}_{X}=\sum_{1 \leq i \leq q} \mathbf{C} \cdot Z_{l}, \quad\left[H, Z_{i}\right]=\lambda_{i} Z_{i}, \quad \lambda_{i} \text { integer } \geq 0 .
$$

It follows from the conjugacy under $G_{Y}$ of the $\mathfrak{I} \mathfrak{l}(2, \mathbf{C})$ 's containing $Y$ that the $\lambda_{l}$ 's are invariants of the orbit $G \cdot Y$. We put

$$
\Lambda(Y)=\Lambda(G \cdot Y)=\sup _{1 \leq i \leq q}\left(\frac{1}{2} \lambda_{i}+1\right)
$$

(c) As a simple application of the above we mention the fact that for any $c \neq 0, c Y$ and $Y$ are in the same $G$-orbit:

$$
c Y \in G \cdot Y, \quad 0 \in \mathrm{Cl}(G \cdot Y) \quad(\mathrm{Cl}=\text { Closure }) .
$$

In fact, as $[H, Y]=-2 Y, Y^{\exp t H}=e^{-2 t} Y$, and we can choose $t$ such that $e^{-2 t}=c$; if $t \in \mathbf{R}$ and $t \rightarrow+\infty, Y^{\exp t H} \rightarrow 0$.

(d) If we regard $G$ as an algebraic group operating linearly on $\mathfrak{g}$, we can conclude that each $G$-orbit in $\mathfrak{g}$ is irreducible and is a Zariski open ( $Z$-open) subset of its closure in the Zariski topology ( $Z$-closure). Moreover, if $\theta$ is the orbit and $\mathrm{Cl}_{Z}(\theta)$ is its Zariski closure, $\mathrm{Cl}_{Z}(\theta) \backslash \theta$ is the union of finitely many orbits each of which has dimension $<\operatorname{dim}(\theta)$. Note that as any orbit is $Z$-open in its $Z$-closure which is irreducible, it makes sense to speak of the dimension of any orbit. Actually these are results valid in the general context of algebraic groups acting on algebraic varieties ([3], p. 98). 
Semisimple orbits are closed, and in fact, they are the only closed ones, either in the complex or the Zariski topologies. In this respect they are very different from the nilpotent orbits (cf. (2.6)).

Let $\Re$ be the set of the nilpotent elements of $g$. Then $\Re$ splits into finitely many orbits ([14]). The classification of the nilpotent orbits is a subtle matter and the reader may consult [24]. We do not use this classification.

If $l=\operatorname{rank}(\mathfrak{g})(l=\operatorname{dim}(V)$ for $\mathfrak{g}=\mathfrak{g l}(V)$; see [30], p. 260), then every orbit in $g$ is of dimension $\leq \operatorname{dim}(g)-l$. An element will be called principal if it generates an orbit of dimension exactly $\operatorname{dim}(g)-l$. Principal semisimple elements are traditionally called regular; they are precisely the elements whose centralizers are Cartan subalgebras of $\mathfrak{g}$. Regular elements of $\mathfrak{g} \mathfrak{l}(V)$ are those endomorphisms of $V$ whose eigenvalues are all simple, i.e., which have, with respect to some basis, diagonal matrices with distinct entries. Principal nilpotents exist and all of them are in one orbit which is necessarily dense and Z-open in $\Re$ (cf. [14], §§1-5, [15], §3).

(e) Since $G$ is a complex group, the $G$-orbits in $g$ may also be regarded as complex manifolds which turn out to be regularly embedded in $\mathfrak{g}$. In fact, let $Z \in \mathrm{g}$ and let $G_{Z}$ be the centralizer of $Z$ in $G$. Write $G^{*}=G / G_{Z}$ and $x \mapsto x^{*}$ for the natural map of $G$ onto $G^{*}$. Since the orbit $G \cdot Z$ is $Z$-open in its $Z$-closure, it is open in its closure (usual topology) and so is locally compact and second countable in its relative topology that it inherits from $g$. Standard arguments then show that $G \cdot Z$ is a regular submanifold of $\mathfrak{g}$ and that the map

$$
\pi_{Z}: x^{*} \mapsto Z^{x}
$$

is a complex analytic isomorphism of $G^{*}$ with $G \cdot Z$.

We shall always identify $g$ with the space of holomorphic left invariant vector fields on $G$ so that the tangent space at every point of $G$ is canonically identified with $\mathfrak{g}$. On the other hand, as $\mathfrak{g}$ is a vector space over C, its complex tangent space at each point is also canonically identified with $g$. It is then clear that the tangent space to the orbit $G \cdot Z$ at $Z$ is $[Z, g]$ :

$$
T_{Z}(G \cdot Z)=[Z, \mathfrak{g}]
$$

For, if $X \in \mathfrak{g}$,

$$
\left(\frac{d}{d z} Z^{\exp z X}\right)_{z=0}=[X, Z]
$$


so that the differential of the map $x \mapsto Z^{x}$ of $G$ onto $G \cdot Z$ at 1 is the linear transformation $X \mapsto[X, Z]$, leading to (2.8). For the dimension of the orbit we have the formula

$$
\operatorname{dim}(G \cdot Z)=\operatorname{dim}([Z, \mathfrak{g}])=\operatorname{dim}(\mathfrak{g})-\operatorname{dim}\left(\mathfrak{g}_{Z}\right) .
$$

In particular,

$$
Z \text { is principal } \Leftrightarrow \operatorname{dim}\left(g_{Z}\right)=l \quad(=\operatorname{rank}(g)) .
$$

If $Y$ is principal nilpotent, $\operatorname{dim}\left(g_{X}\right)=l$ and the $\lambda_{l}$ in $(2.4)$ are even integers ([14], [28]).

In case $\mathfrak{g}=\mathfrak{g} \mathfrak{l}(n, \mathbf{C})$, we have $\mathfrak{g}=\mathbf{C} \cdot 1 \oplus \mathfrak{g} \mathfrak{l}(n, \mathbf{C})$ and $\mathfrak{g}_{X}=\mathbf{C} \cdot 1 \oplus$ $\left(g_{X} \cap \mathfrak{g l}(n, \mathbf{C})\right)$. It is often convenient to assume that the $Z_{l}$ in (2.4) are chosen so that

$$
Z_{1}=1, Z_{2}, \ldots, Z_{q} \in \mathfrak{g}_{X} \cap \mathfrak{g} \mathfrak{I}(n, \mathbf{C}) .
$$

In particular $\lambda_{1}=0$. A typical choice of principal nilpotent is

$$
Y_{n}=\left(\begin{array}{ccccc}
0 & & & & \\
b_{1} & 0 & & \bigcirc & \\
0 & b_{2} & 0 & \cdots & \\
\vdots & \vdots & & \ddots & \\
0 & 0 & \cdots & b_{n-1} & 0
\end{array}\right)
$$

where

$$
b_{j}=j(n-j), \quad 1 \leq j \leq n-1 .
$$

A simple calculation shows that if we take

$$
\begin{aligned}
& H_{n}=\left(\begin{array}{cccc}
n-1 & & & 0 \\
& n-3 & \ddots & -(n-1)
\end{array}\right) \text {, } \\
& X_{n}=\left(\begin{array}{ccccc}
0 & 1 & 0 & \cdots & 0 \\
& 0 & 1 & & 0 \\
& & \ddots & & 1 \\
& 0 & & & 0
\end{array}\right)
\end{aligned}
$$


then

$$
\left\{H_{n}, X_{n}, Y_{n}\right\} \text { is a standard triple. }
$$

So $X_{n}$ is a nilpotent opposite to $Y_{n}$; the choices (2.15) are thus seen to be made so that $X_{n}$ takes a simple form. The centralizer of $X_{n}$ is seen to be the space of all upper triangular matrices whose elements are constant on each diagonal (companion matrices, see [8]):

$$
Z \in \mathfrak{g}_{X_{n}} \Leftrightarrow Z=\left(\begin{array}{ccccc}
a_{1} & a_{2} & \cdots & a_{n-1} & a_{n} \\
0 & a_{1} & a_{2} & \cdots & a_{n-1} \\
& & \ddots & & \\
& 0 & & a_{1} & a_{2} \\
& & & & a_{1}
\end{array}\right) .
$$

Let $E_{i j}(1 \leq i, j \leq n)$ be the matrix units, i.e., $E_{i j}$ is the matrix which has 1 in its $i j$ th cell and 0 in all others. Define

$$
Z_{i}=E_{1, i}+E_{2, i+1}+\cdots+E_{n-i+1, n} \quad(1 \leq i \leq n) .
$$

Then

$$
Z_{1}=1, \quad \mathfrak{g}_{X_{n}}=\mathbf{C} \cdot Z_{1}+\cdots+\mathbf{C} \cdot Z_{n}
$$

Moreover

$$
\left[H_{n}, Z_{i}\right]=2(i-1) Z_{i} \quad(1 \leq i \leq n) .
$$

It is immediately evident from (2.18) that $\Lambda\left(Y_{n}\right)=n$.

We shall now obtain an estimate for $\Lambda(Y)$. Let $g$ be arbitary reductive, $Y \neq 0$ a nilpotent, and $\{H, X, Y\}$ a standard triple. If the integers $\lambda_{l}$ are as in (2.4), it is easily seen that $\sup _{1 \leq i \leq q} \lambda_{i}$ is the largest eigenvalue of ad $H$. On the other hand, let $\mathfrak{h}$ be a Cartan subalgebra containing $H$ and let us choose a positive system of roots of $(\mathfrak{g}, \mathfrak{h})$ such that $\alpha(H) \geq 0$ for all positive roots $\alpha$. Let $\alpha_{1}, \ldots, \alpha_{l}$ be the simple roots. It is known that $\alpha_{i}(H) \in\{0,1,2\}$ for all $i=1, \ldots, l$, and that for principal $Y, \alpha_{i}(H)=2$ for all $i=1, \ldots, l$ (cf. [14], Lemmas 5.1 and 5.2). So, if $\Delta_{1}$ (resp. $\Delta_{2}$ ) is the set of $i$ for which $\alpha_{i}(H)=1\left(\operatorname{resp} . \alpha_{l}(H)=2\right)$, then

$$
\Lambda(Y)=1+\frac{1}{2} \max \left(\sum_{i \in \Delta_{1}} m_{i}+2 \sum_{j \in \Delta_{2}} m_{j}\right)
$$

where the maximum is over all $m_{1}, \ldots, m_{l}$ such that $\alpha=m_{1} \alpha_{1}+\cdots+m_{l} \alpha_{l}$ is a positive root. 
For any positive root $\alpha=m_{1} \alpha_{1}+\cdots+m_{l} \alpha$, we call $m_{1}+\cdots+m_{l}$ the order of $\alpha$. Write

(2.20) $\operatorname{ord}(\mathfrak{g})=$ maximum of the orders of the positive roots of $\mathfrak{g}$.

Obviously ord $(g)$ is the maximum of the orders of the highest roots of the simple factors of $\mathrm{g}$. From (2.19) we easily obtain the following.

Proposition 1. For any nilpotent $Y \neq 0, \Lambda(Y) \leq 1+\operatorname{ord}(\mathrm{g})$; if $Y$ is principal, $\Lambda(Y)=1+\operatorname{ord}(g)$.

For $\mathfrak{g}=\mathfrak{g l}(n, \mathbf{C})$, the roots are $\pm\left(\alpha_{l}+\alpha_{t+1}+\cdots+\alpha_{\jmath}\right), 1 \leq i \leq j \leq$ $n-1$, so that $\operatorname{ord}(\mathfrak{g})=n-1$. So $\Lambda(Y) \leq n$ in this case.

2.2. The following results are of fundamental importance for us.

Proposition 1. Let $Y \neq 0$ be a nilpotent of $\mathfrak{g}$. Let $X, H$ be in $[\mathfrak{g}, \mathfrak{g}]$ such that $\{X, H, Y\}$ is a standard triple. Let $\mathrm{g}_{X}$ be the centralizer of $X$ in $\mathfrak{g}$. Then the affine space $Y+\mathrm{g}_{X}$ meets the orbit $G \cdot Y$ exactly at $Y$.

Proof. We establish first that for a suitable open neighborhood $\mathfrak{n}_{X}$ of 0 in $\mathfrak{g}_{X}$, the orbit $G \cdot Y$ meets $Y+\mathfrak{n}_{X}$ only at $Y$. Let $G^{*}=G / G_{Y}$ and $x \mapsto x^{*}$ the natural map of $G$ onto $G^{*}$. We consider the map

$$
\psi: x^{*}, Z \mapsto Y^{x}+Z \quad\left(x^{*} \in G^{*}, Z \in g_{X}\right) .
$$

It follows from (2.9) that the differential $(d \psi)_{\left(1^{*}, 0\right)}$ has for its range $[Y, \mathfrak{g}]+\mathfrak{g}_{X}$ which is $\mathfrak{g}$ by $(2.3)$. Hence $(d \psi)_{\left(1^{*}, 0\right)}$ is a linear isomorphism. So there are open neighborhoods $U^{*}$ of $1^{*}$ in $G^{*}$, and $n_{X}^{\prime}$ of 0 in $g_{X}$, such that $\psi$ is an analytic isomorphism of $U^{*} \times n_{X}^{\prime}$ with an open neighborhood $\mathfrak{n}$ of $Y$ in $\mathrm{g}$. We claim that if $\mathrm{n}_{X}$ is a sufficiently small open neighborhood of 0 in $\mathfrak{n}_{X}^{\prime}$, the orbit $G \cdot Y$ meets $Y+\mathfrak{n}_{X}$ only at $Y$. If this is not true, we can find a sequence $\left(x_{n}\right)_{n \geq 1}$ in $G$ such that

$$
Y^{x_{n}}=Y-Z_{n}, \quad 0 \neq Z_{n} \in \mathrm{g}_{X}, \quad Z_{n} \rightarrow 0 .
$$

Now we have already remarked in $\S 2.1$ that the map $x^{*} \mapsto Y^{x}$ is a homeomorphism of $G^{*}$ with $G \cdot Y$, the latter being given the topology induced by $g$. So, as $Y^{x_{n}} \rightarrow Y$, we have $x_{n}^{*} \rightarrow 1^{*}$. Hence $x_{n}^{*} \in U^{*}$ for all $n \geq n_{0}$. We may also assume that $Z_{n} \in \mathfrak{n}_{X}^{\prime}$ and $Y^{x_{n}} \in \mathfrak{n}$ for $n \geq n_{0}$. But then, for $n \geq n_{0}$,

$$
\psi\left(x_{n}^{*}, Z_{n}\right)=Y^{x_{n}}+Z_{n}=Y=\psi\left(1^{*}, 0\right)
$$


giving $x_{n}^{*}=1^{*}, Z_{n}=0$, as $\psi$ is one-one on $U^{*} \times \mathfrak{n}_{X}^{\prime}$. As $Z_{n} \neq 0$ we have a contradiction. Our claim is thus established.

In order to prove the proposition we need therefore only show that if the orbit $G \cdot Y$ meets $Y+\mathrm{g}_{X}$ at points other than $Y$, it meets it also at points different from, but arbitrarily close to, $Y$. Let $\left(Z_{i}\right)_{1 \leq i \leq q}$ be a basis of $\mathfrak{g}_{X}$ satisfying (2.4). Suppose $\left(c_{1}, \ldots, c_{q}\right) \neq(0, \ldots, 0)$ is such that

$$
U=Y+c_{1} Z_{1}+\cdots+c_{q} Z_{q} \in G \cdot Y .
$$

Then

$$
U^{\exp t H}=e^{-2 t} Y+c_{1} e^{\lambda_{1} t} Z_{1}+\cdots+c_{q} e^{\lambda_{q} t} Z_{q} \in G \cdot Y
$$

for all $t \in \mathbf{C}$. Let $V_{t}=e^{2 t} U^{\exp t H}$. As $U^{\exp t H} \in G \cdot Y$, it is nilpotent and so is conjugate to any nonzero multiple of it, by (2.6). Hence $V_{t} \in G \cdot Y$ also, for all $t \in \mathbf{C}$. But now,

$$
V_{t}=Y+c_{1} e^{\left(\lambda_{1}+2\right) t} Z_{1}+\cdots+c_{q} e^{\left(\lambda_{q}+2\right) t} Z_{q} .
$$

Since all $\lambda_{i}$ are $\geq 0$, the exponentials above tend to 0 when $t \in \mathbf{R}$ and goes to $-\infty$. Hence

$$
\begin{aligned}
V_{t} \in G \cdot Y, \quad V_{t} \neq Y, \quad V_{t} \in Y+\mathfrak{g}_{X}, \quad V_{t} \rightarrow Y \\
(t \rightarrow-\infty \text { through real values })
\end{aligned}
$$

as we wanted.

Proposition 2. If $U \in Y+\mathrm{g}_{X}$ is nilpotent and $U \neq Y$ then $\operatorname{dim}(G \cdot U)>\operatorname{dim}(G \cdot Y)$.

Proof. Let $U=Y+c_{1} Z_{1}+\cdots+c_{q} Z_{q}, Z_{t}$ as in (2.4). Define $V_{t}$ as before. Then $V_{t} \rightarrow Y$ as $t \rightarrow-\infty$ through real values. Since $U$ is nilpotent, (2.6) implies that $V_{t} \in G \cdot U$. Hence $Y \in \mathrm{Cl}(G \cdot U)$, implying that $Y$ is in the $Z$-closure of the orbit of $U$. By $\$ 2.1(\mathrm{~d})$, we have $\operatorname{dim}(G \cdot U) \geq$ $\operatorname{dim}(G \cdot Y)$ with equality only if $Y \in G \cdot U$, i.e., if $U \in G \cdot Y$. But $G \cdot Y$ meets $Y+\mathfrak{g}_{X}$ only at $Y$. Hence, as $U \neq Y$, we cannot have $U \in G \cdot Y$. So we must have $\operatorname{dim}(G \cdot U)>\operatorname{dim}(G \cdot Y)$.

COROllaRY 1. If $Y$ is a principal nilpotent, it is the only nilpotent in $Y+\mathfrak{g}_{X} \cdot$

Proof. For $\operatorname{dim}(G \cdot Y)$ has the maximum possible dimension.

For non-nilpotent elements in $Y+\mathfrak{g}_{X}$ we have

Proposition 3. For any $U \in Y+\mathfrak{g}_{X}, \operatorname{dim}(G \cdot U) \geq \operatorname{dim}(G \cdot Y)$. 
We do not prove this here since we make no use of it.

\section{The Classical Connections}

\section{Reduction theory in the regular case.}

3.1. The reduction theory of regular connections is very well known and so we limit ourselves to a very brief review for later use. The main result is that they are determined upto $\operatorname{GL}(V(\mathscr{F}))$-equivalence by their monodromy. This comes out as a byproduct of the determination of the orbit space $\operatorname{GL}(V(\theta))_{1} \backslash \Omega$ where $\Omega$ is the space of connections of the first kind, i.e., of $A \in \mathfrak{g} \mathfrak{l}(V(\mathscr{F}))$ with $\operatorname{ord}(A) \geq-1$. The orbits are parametrized by the so-called aligned models.

The study of $\Omega$ as a $\operatorname{GL}(V(\theta))_{1}$-space goes back already to Birkhoff [2] (although he erroneously asserted that every element of $\Omega$ can be transformed to $z^{-1} A_{-1}$ under $\left.\operatorname{GL}(V(\theta))_{1}\right)$. The reduction to an aligned model is known (see Gantmacher ([9], p. 162)) and also Turrittin ([26], p. $33)$. We sharpen this result by proving that the aligned model of an element of $\Omega$ is determined upto conjugacy by a suitable finite dimensional subgroup of $\mathrm{GL}(V(\theta))_{1}$.

\subsection{Let $A \in \Omega$ be given by}

$$
A=z^{-1} A_{-1}+A_{0}+z A_{1}+\cdots+z^{m} A_{m}+\cdots .
$$

We write $\sigma=\sigma\left(A_{-1}\right)$ for the set of eigenvalues of $A_{-1}$. For $\lambda \in \sigma$ let $V_{\lambda}$ be the corresponding spectral subspace of $A_{-1}$, i.e., the largest subspace of $V$ stable under $A_{-1}$ on which $A_{-1}-\lambda \cdot 1$ is nilpotent. Let $P_{\lambda}\left(V \rightarrow V_{\lambda}\right)$ be the projections corresponding to the direct sum $V=\bigoplus_{\mu \in \sigma} V_{\mu}$. If $m$ is an integer $\geq 0$ and $(\lambda, \mu) \in \sigma \times \sigma$, we say

$$
m \text { is aligned with }(\lambda, \mu) \text { if } \lambda-\mu=m+1 .
$$

Proposition. Let $A$ be as in (3.1). Then we can find $x$ in $\operatorname{GL}(V(\theta))_{1}$ such that $B=x[A]=z^{-1} B_{-1}+B_{0}+z B_{1}+\cdots$ has the following properties:

(a) $B_{-1}=A_{-1}$.

(b) For $m \geq 0,(\lambda, \mu) \in \sigma \times \sigma, P_{\lambda} B_{m} P_{\mu}=0$ if $m$ is not aligned with $(\lambda, \mu)$.

Proof. Let $x=1+z T_{1}+z^{2} T_{2}+\cdots$ where $T_{j}$ is to be chosen so that (a) and (b) are satisfied for $B=x[A]$. Since $x A+\dot{x}=B x$, we get 
$A_{-1}=B_{-1}$, and, for $m \geq 0$

$$
\begin{aligned}
B_{m}= & A_{m}+\sum_{1 \leq r \leq m}\left(T_{r} A_{m-r}-B_{m-r} T_{r}\right) \\
& -\left(\operatorname{ad}\left(A_{-1}\right)-(m+1)\right)\left(T_{m+1}\right) .
\end{aligned}
$$

For $(\lambda, \mu) \in \sigma \times \sigma$ and any endomorphism $L$ of $V$ we write $L_{\lambda \mu}$ for $P_{\lambda} L P_{\mu}$ and regard it in a natural way as an element of $\operatorname{Hom}_{\mathbf{C}}\left(V_{\mu}, V_{\lambda}\right)$. Since $V_{\lambda}$ and $V_{\mu}$ are stable under $A_{-1}$ we have

$$
\left(\operatorname{ad}\left(A_{-1}\right)\left(T_{m+1}\right)\right)_{\lambda \mu}=\operatorname{ad}\left(A_{-1}\right)\left(\left(T_{m+1}\right)_{\lambda \mu}\right) .
$$

Hence (3.3) leads to

$$
\begin{aligned}
\left(B_{m}\right)_{\lambda \mu}= & \left(A_{m}\right)_{\lambda \mu}+\sum_{1 \leq r \leq m}\left(T_{r} A_{m-r}-B_{m-r} T_{r}\right)_{\lambda \mu} \\
& -\left(\operatorname{ad}\left(A_{-1}\right)-(m+1) 1\right)\left(\left(T_{m+1}\right)_{\lambda \mu}\right)
\end{aligned}
$$

for all $(\lambda, \mu) \in \sigma \times \sigma$. Now $\operatorname{ad}\left(A_{-1}\right)-(m+1) 1$ has $\lambda-\mu-(m+1)$ as its sole eigenvalue on $\operatorname{Hom}_{\mathbf{C}}\left(V_{\mu}, V_{\lambda}\right)$. So, if $B_{j}(j \leq m-1), T_{k}(k \leq m)$ have already been defined, we can define $B_{m}$ and $T_{m+1}$ as follows: if $m$ is not aligned with $(\lambda, \mu), \operatorname{ad}\left(A_{-1}\right)-(m+1) 1$ is invertible on $\operatorname{Hom}_{\mathbf{C}}\left(V_{\mu}, V_{\lambda}\right)$ and so we choose $\left(T_{m+1}\right)_{\lambda \mu}$ so that (3.4) is true with $\left(B_{m}\right)_{\lambda \mu}=0$; if $m$ is aligned with $(\lambda, \mu)$, we choose $\left(T_{m+1}\right)_{\lambda \mu}=0$ and define $\left(B_{m}\right)_{\lambda \mu}$ by (3.4). This completes the definition of $B_{m}$ and $T_{m+1}$ and the proposition is proved by induction on $m$.

Any $B$ as in the above Proposition is said to be an aligned model for A.

3.3. We shall now examine the extent of non-uniqueness of the aligned model. Let $X \in \mathfrak{g l}(V)$, let $\Omega(X)$ be the set of all $A \in \Omega$ with $A_{-1}=X$, and let $\Re=\Re_{(}(X)$ be the set of all $B \in \Omega$ satisfying (a) (with $A_{-1}=X$ ) and (b) of Proposition 3.2. Let

$$
\begin{aligned}
g(X)=\mathscr{g}=\left\{x=1+z T_{1}+\cdots\right. & \mid\left(T_{m}\right)_{\lambda \mu}=0 \\
& \text { unless } \lambda-\mu=m\} .
\end{aligned}
$$

Then $g$ is a subgroup of $\operatorname{GL}(V(\theta))_{1}$ and $x \mapsto x(1)$ (which is well-defined) is an isomorphism of $q$ with the subgroup $U$ of $\operatorname{GL}(V)$ given by

$$
\begin{aligned}
U=\left\{u \in \mathrm{GL}(V) \mid u_{\lambda \lambda}\right. & =1, u_{\lambda \mu}=0 \\
& \text { unless } \lambda-\mu \text { is an integer } \geq 0\} .
\end{aligned}
$$


Finally, let $\mathfrak{N}=\mathfrak{R}(X)$ be the affine subspace of all $N \in \mathfrak{g l}(V)$ such that (3.7) $\quad N_{\lambda \lambda}=\left(\left(A_{-1}\right)_{n}\right)_{\lambda \lambda}, \quad N_{\lambda \mu}=0 \quad$ unless $\lambda-\mu$ is an integer $\geq 0$.

For any $B \in \Re$ let

$$
\tilde{B}=B(1)-X_{s}
$$

where, as usual, $X_{s}$ is the semisimple component of $X$. Obviously the map $B \mapsto \tilde{B}$ is a bijection of $\mathscr{B}$ with $\mathfrak{R}$.

LEMMA 1. Gacts on $\Re$. If $B \in \Re, x \in \mathcal{G}$ and $B^{\prime}=x[B]$, then

$$
\tilde{B}^{\prime}=x(1) \tilde{B} x(1)^{-1} \text {. }
$$

Proof. This is a straightforward verification.

Lemma 2. Let $B, B^{\prime} \in \mathscr{B}$ and $x \in \mathrm{GL}(V(\theta))_{1}$ such that $x[B]=B^{\prime}$. Then $x \in \mathcal{G}$.

Proof. Let $x=1+z T_{1}+\cdots$. Now, we have, for all integers $m \geq 0$, $(\lambda, \mu) \in \sigma \times \sigma$,

$$
\begin{aligned}
\left(B_{m}^{\prime}\right)_{\lambda \mu}= & \left(B_{m}\right)_{\lambda \mu}+\sum_{1}^{r}\left[\left(T_{r} B_{m-r}\right)_{\lambda \mu}-\left(B_{m-r}^{\prime} T_{r}\right)_{\lambda \mu}\right] \\
& -(\operatorname{ad}(X)-(m+1) 1)\left(\left(T_{m+1}\right)_{\lambda \mu}\right)
\end{aligned}
$$

Assume that, for $j \leq m,\left(T_{j}\right)_{\lambda \mu}=0$ unless $\lambda-\mu=j$. Suppose $\lambda-\mu \neq$ $m+1$. Then $m$ is not aligned to $(\lambda, \mu)$ and so, $\left(B_{m}\right)_{\lambda \mu}=0,\left(B_{m}^{\prime}\right)_{\lambda \mu}=0$. On the other hand, for $1 \leq r \leq m$,

$$
\left(T_{r} B_{m-r}\right)_{\lambda \mu}=\sum_{\nu}\left(T_{r}\right)_{\lambda \nu}\left(B_{m-r}\right)_{\nu \mu} .
$$

If both $\left(T_{r}\right)_{\lambda \nu}$ and $\left(B_{m-r}\right)_{\nu \mu}$ are nonzero for some $\nu$, we must have $\lambda-\nu=r$ (by the induction hypothesis) and $\nu-\mu=m-r+1$ (as $B \in$ $\mathscr{B}(X))$. Hence $\lambda-\mu=m+1$, which contradicts our assumption. Hence $\left(T_{r} B_{m-r}\right)_{\lambda \mu}=0$. Similarly we show $\left(B_{m-r}^{\prime} T_{r}\right)_{\lambda \mu}=0$. Thus $(\operatorname{ad}(X)-(m+1) 1)\left(\left(T_{m+1}\right)_{\lambda \mu}\right)=0$. As $\lambda-\mu \neq m+1$, we must have $\left(T_{m+1}\right)_{\lambda \mu}=0$, as we wanted to prove. 
We have thus obtained

Theorem 1. Fix $X \in \mathfrak{g} \mathfrak{l}(V)$. For each $A \in \Omega(X)$ let $B$ be an aligned model for $A$, and let $\tilde{B}$ be as in (3.8). Then $B$ (resp. $\tilde{B}$ ) is unique upto conjugacy by $\mathscr{G}$ (resp. $U$ ); and the map that assigns to the $\mathrm{GL}(V(\theta))_{1}$-equivalence class of $A$ the $U$-orbit of $\tilde{B}$ is well defined and determines a bijection:

$$
\mathrm{GL}(V(\theta))_{1} \backslash \Omega \stackrel{\sim}{\rightarrow} U \backslash \mathfrak{R} .
$$

We give a few examples to convince the reader that the structure of $U \backslash \mathfrak{R}$, and hence that of $\operatorname{GL}(V(\theta))_{1} \backslash \Omega$, is not simple. The notation is as in Theorem 1.

(1) No two distinct eigenvalues of $A_{-1}$ differ by an integer. Here $\mathfrak{N}$ consists of a single element bl.diag $\left(N_{1}, \ldots, N_{p}\right)\left(N_{i}\right.$ nilpotent $)$, and $U=(1)$. Hence there is only one orbit, and we have

$$
x[A]=z^{-1} A_{-1}
$$

for some $x \in \mathrm{GL}(V(\theta))_{1}$. Actually the proof of the Proposition 3.2 gives an algorithm for determining one such $x$.

(2) All eigenvalues of $A_{-1}$ are simple and any two of them differ by an integer. This is the other extreme type of example. $\mathfrak{N}$ is the Lie algebra of lower triangular nilpotent matrices and $U$ is its adjoint group. The space $U \backslash \mathfrak{R}$ has been studied in considerable detail (see [21] for instance). The aligned model $z^{-1} A_{-1}$ corresponds to the orbit (0).

3.4. Let $A \in \Omega$ be as in (1). Write the eigenvalues of $A_{-1}$ as $\left\{\lambda_{i j} \mid 1 \leq\right.$ $\left.i \leq p, 1 \leq j \leq n_{i}\right\}$ where $\lambda_{i j}-\lambda_{i^{\prime} j^{\prime}} \in \mathbf{Z}$ if and only if $i=i^{\prime}$, and $\lambda_{i j}$ $\lambda_{i j^{\prime}}>0$ if $j>j^{\prime}$.

Let $B$ be an aligned model for $A$. Let $u \in \mathrm{GL}\left(V\left(\mathscr{F}_{\text {cgt }}\right)\right)$ be defined by $u_{\lambda \mu}=0$ for $\lambda \neq \mu$ and, for $\lambda=\lambda_{l j}=\mu, u_{\lambda \mu}=z^{\lambda_{t 1}-\lambda_{t \jmath}} \cdot 1,1 \leq i \leq p, 1 \leq j$ $\leq n_{l}$.

Proposition. With $u$ as above, we have

$$
u[B]=z^{-1} C
$$

where $C=\tilde{B}+\Lambda$ and $\Lambda$ acts as the scalar $\lambda_{i 1}$ on all the spectral subspaces $V_{\lambda}$ for $\lambda=\lambda_{i j}, 1 \leq j \leq n_{i}$.

Proof. Straightforward calculation.

REMARK. By arguing as in the proof of Proposition 1.7.2 it is easy to see that $\operatorname{lag}(u)=\max _{i}\left|\lambda_{t 1}-\lambda_{t n_{t}}\right|$. This will be important in $\S 6.6$. 
3.5. We say that an endomorphism $L$ of $V$ is reduced if

$$
\text { the real parts of all eigenvalues of } L \text { lie in }[0,1) \text {. }
$$

The property of being reduced is invariant under the action of $\mathrm{GL}(V)$, and so it makes sense to speak of reduced $\mathrm{GL}(V)$-orbits in $g \mathfrak{l}(V)$.

For any $\lambda \in \mathbf{C}$ let $(\lambda)$ be the element of $\mathbf{C}$ defined uniquely by $0 \leq \operatorname{Re}((\lambda))<1, \lambda-(\lambda) \in \mathbf{Z}$. If $C$ is any endomorphism of $V$ with spectrum $\sigma(C)$ and spectral projections $Q_{\lambda}(\lambda \in \sigma(C))$, we define

$$
C_{\mathrm{red}}=\sum_{\lambda \in \sigma(C)}(\lambda) Q_{\lambda}, \quad x=\sum z^{(\lambda)-\lambda} Q_{\lambda} .
$$

It is obvious that

$$
\exp 2 \pi i C=\exp 2 \pi i C_{\mathrm{red}}, \quad x\left[z^{-1} C\right]=z^{-1} C_{\mathrm{red}} .
$$

LEMMA. The exponential map induces a bijection from the set of reduced $\mathrm{GL}(V)$-orbits in $\mathrm{gl}(V)$ to the set of all conjugacy classes in $\mathrm{GL}(V)$.

Proof. It is well known that the exponential map from $\mathfrak{g l}(V)$ to $\mathrm{GL}(V)$ is surjective. The lemma is an immediate consequence of this, (3.11), and the obvious fact that if $C$ is reduced, the spectral projections of $\exp 2 \pi i C$ are precisely the $Q_{\lambda}$ corresponding to $e^{2 \pi i \lambda}$.

3.6. We say $A \in \mathfrak{g l}(V(\mathscr{F}))$ is regular if there exists $x \in \mathrm{GL}(V(\mathscr{F}))$ such that $x[A]$ is of the first kind. We see from $\S \S 3.2,3.4$ and (3.11) that a regular $A$ is equivalent over $\operatorname{GL}(V(\mathscr{F}))$ to $z^{-1} C$ for some reduced $C \in$ $\mathfrak{g} \mathfrak{l}(V)$.

Theorem. For any regular $A \in \mathfrak{g l}(V(\mathscr{F}))$ let $C \in \mathfrak{g l}(V)$ (resp. reduced

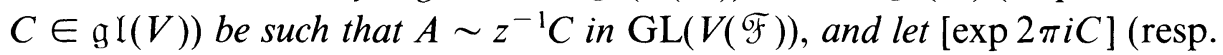
$[C])$ be the conjugacy class of $\exp 2 \pi i C$ (resp. $C$ ) in $\mathrm{GL}(V)$ (resp. $\mathfrak{g} \mathfrak{l}(V)$ ). Then $[\exp 2 \pi i C]$ (resp. $[C])$ is uniquely determined by $A$, depends only on the $\operatorname{GL}(V(\mathcal{F}))$-equivalence class $[A]$ of $A$, and the map

$$
[A] \mapsto[\exp 2 \pi i C] \quad(\operatorname{resp} .[A] \rightarrow[C])
$$

is a bijection of the set of regular $\operatorname{GL}(V(\mathscr{F}))$-equivalence classes in $\mathfrak{g} l(V(\mathscr{F}))$ with the set of conjugacy classes in $\mathrm{GL}(V)$ (resp. reduced conjugacy classes in $\mathfrak{g} \mathfrak{l}(V))$.

The theorem is immediate from our discussion so far and the following lemma. 
Lemma. Suppose $C_{1}, C_{2} \in \mathfrak{g l}(V)$ are reduced, and $t \in \mathrm{GL}(V(\mathscr{F}))$ is such that $t\left[z^{-1} C_{1}\right]=z^{-1} C_{2}$. Then $t$ lies in $\mathrm{GL}(V)$ and $t C_{1} t^{-1}=C_{2}$.

Proof. Let us write $t$ in the form

$$
t=\sum_{k>-\infty} t_{k} z^{k}
$$

The equation $t\left(z^{-1} C_{1}\right)+\dot{t}=\left(z^{-1} C_{2}\right) t$ then leads to the relations

$$
t_{k} C_{1}+k t_{k}=C_{2} t_{k} .
$$

Let $L$ be the endomorphism of $\mathfrak{g l}(V)$ defined by $L(u)=C_{2} u-u C_{1}$ $\left(u \in \mathfrak{g l}(V)\right.$ ). Then the eigenvalues of $L$ are of the form $\lambda_{2}-\lambda_{1}$ where $\lambda_{t}$ runs through the eigenvalues of $C_{i}, i=1,2$. As $C_{1}$ and $C_{2}$ are reduced, we see that $|\operatorname{Re}(\lambda)|<1$ for all eigenvalues $\lambda$ of $L$. Rewriting (3.13) as $L\left(t_{k}\right)=k t_{k}$ we conclude that $t_{k}=0$ if $k \neq 0$.

3.7. If $A \in \mathfrak{g l}(V(\mathscr{F}))$ is regular and $\sim z^{-1} C$ under $\operatorname{GL}(V(\mathscr{F}))$ where $C \in \mathfrak{g l}(V)$, we call [ $\exp 2 \pi i C$ ], the $\mathrm{GL}(V)$-conjugacy class of $\exp 2 \pi i C$, the monodromy of $A$. Let $A$ be as in (3.1), $B$ an aligned model for $A$, and $\tilde{B}$ as in (3.8).

THEOREM. The monodromy of $A$ is $[\gamma]$ where $\gamma=\exp 2 \pi i\left(A_{-1}\right)_{s}$. $\exp 2 \pi i \tilde{B}$. Moreover, this is the Jordan decomposition of $\gamma$.

Proof. This is immediate from Proposition 3.4.

COROLlary 1. The elements in the monodromy (class) of $A$ have the same characteristic polynomial as $\exp 2 \pi i A_{-1}$.

3.8. Starting with $A$ as in (3.1), the determination of its monodromy is thus seen to depend on the determination of $B$ in Proposition 3.2 whose proof gives a computational procedure for doing this. It is moreover clear from that proof that the $B_{m}$ are determined recursively and that $B_{m}$ depends only on $A_{-1}, A_{0}, \ldots, A_{m}$. Let

$$
k\left(A_{-1}\right)=\max _{1 \leq j \leq p} \max _{1 \leq r \leq n_{j}}\left(\lambda_{j r}-\lambda_{j 1}-1\right) .
$$

It is then immediate that for $m>k\left(A_{-1}\right), m$ is not aligned with any $(\lambda, \mu)$ in $\sigma \times \sigma$, so that $B_{m}=0$. So $B$ is determined by a knowledge of the 
$B_{s}, s \leq k\left(A_{-1}\right)$, and hence by a knowledge of the $A_{s}, s \leq k\left(A_{-1}\right)$. We thus obtain

Proposition. Let $A$ be as in (3.1). Then its monodromy is already determined by the coefficients $A_{s}, s \leq k\left(A_{-1}\right), k\left(A_{-1}\right)$ being the integer $\geq-1$ defined by (3.14).

The bound $k\left(A_{-1}\right)$ obtained above is in fact sharp, as may be seen from the example with

$$
A=\left(\begin{array}{cc}
0 & 0 \\
\alpha z^{m-1} & m z^{-1}
\end{array}\right), \quad \alpha \neq 0, m \text { integer } \geq 1 .
$$

Then $k\left(A_{-1}\right)=m-1$ and the monodromy is

$$
\left[\left(\begin{array}{cc}
1 & 0 \\
e^{2 \pi l \alpha} & 1
\end{array}\right)\right]
$$

3.9. It is obviously of interest to know when the monodromy is the conjugacy class [ $\exp 2 \pi i A_{-1}$ ]. This will be so if $\tilde{B}=\left(A_{-1}\right)_{n}$. The classical sufficient condition for this is that no two (distinct) eigenvalues of $A_{-1}$ differ by an integer. By scrutinizing the recursion formulae (3.4) more carefully one can prove the following sharpened form of it. We omit the proof.

Proposition. Suppose that the coefficients $A_{m}$ satisfy either the conditions

$$
A_{m}=0 \quad \text { on } \bigoplus_{1 \leq i \leq p} \bigoplus_{1 \leq r<n_{\imath}} V_{\lambda_{t r}} \text { for } 0 \leq m \leq k\left(A_{-1}\right)
$$

or the dual conditions

$$
\operatorname{range}\left(A_{m}\right) \subset \bigoplus_{1 \leq i \leq p} V_{\lambda_{t 1}} \text { for } 0 \leq m \leq k\left(A_{-1}\right) .
$$

Then $A$ is equivalent to $z^{-1} A_{-1}$ over $\mathrm{GL}(V(\theta))_{1}$.

3.10. In $\S 6$ we shall be interested in the question of when two elements $R_{1}$ and $R_{2}$ which are regular in $\mathfrak{g l}(V(\mathscr{F}))$ are equivalent with respect to $\operatorname{GL}(V(\overline{\mathscr{F}}))$.

Proposition 1. Suppose $R_{1}$ and $R_{2}$ in $g \mathfrak{l}(V(\widetilde{F}))$ are regular. Then they are $\operatorname{GL}(V(\overline{\mathscr{F}}))$-equivalent if and only if for some integer $k \geq 1$, the kth powers of their monodromies are the same. 
Proof. We may clearly assume that $R_{J}=z^{-1} C_{J}, j=1,2$, so that $M_{j}=\exp 2 \pi i C_{j}$ defines the monodromy of $R_{j}$. If $d \geq 1$ is an integer, it is immediate by going over to the $\zeta$-liftings that $R_{1}$ and $R_{2}$ are equivalent under $\operatorname{GL}\left(V\left(\mathscr{F}_{d}\right)\right)$ if and only if $\zeta^{-1}\left(d C_{1}\right)$ and $\zeta^{-1}\left(d C_{2}\right)$ are equivalent in $\operatorname{GL}\left(V\left(\mathscr{F}_{\xi}\right)\right)$, i.e., if and only if $\left[M_{1}^{d}\right]=\left[M_{2}^{d}\right]$.

There is also an $\overline{\mathscr{F}}$-version of Proposition 3.8 which we will need in $\S 6.6$. Let $C \in \mathfrak{g I}(V)$ and let $\sigma(C)$ be the set of its eigenvalues. Then we can describe $\sigma(C)$ as

$$
\sigma(C)=\left\{\lambda_{l j} \mid 1 \leq i \leq p, 1 \leq j \leq n_{\imath}\right\}
$$

where for $i \neq i^{\prime}, \lambda_{l j}-\lambda_{i^{\prime} j^{\prime}} \notin \mathbf{Q}$ while $\lambda_{l j}-\lambda_{i j^{\prime}}$ is $>0$ for $j>j^{\prime}$. Let

$$
\bar{k}(C)=\max _{1 \leq i \leq p}\left(\lambda_{i n_{i}}-\lambda_{i 1}\right) \text {. }
$$

Proposition 2. Fix $C \in \mathfrak{g} l(V)$ and write $\bar{k}=\bar{k}(C)$. Suppose $A, B \in$ $\operatorname{gl}(V(\overline{\mathscr{F}}))$ are of the first kind and $A_{-1}=B_{-1}=C$. If $A \equiv B\left(\bmod z^{\bar{k}}\right)$, then $A$ and $B$ are equivalent in $\operatorname{GL}(V(\overline{\mathscr{F}}))$.

Proof. Let $d \geq 1$ be an integer such that $A, B \in \mathfrak{g l}\left(V\left(\mathscr{F}_{d}\right)\right)$ and all $\lambda_{i j}-\lambda_{i j^{\prime}} \in(1 / d) \mathbf{Z}$. The result follows by going over to $\zeta=z^{1 / d}$ and using Proposition 3.8.

3.11. We close this section with a brief discussion of the convergent case. For any subset $\Gamma$ of $\mathfrak{g l}(V(\mathscr{F}))$ we put $\Gamma_{\text {cgt }}=\Gamma \cap \mathfrak{g l}\left(\mathrm{V}\left(\mathscr{F}_{\text {cgt }}\right)\right)$.

It is obvious that if $A \in \mathfrak{g} \mathfrak{l}\left(V\left(\mathscr{F}_{\text {cgt }}\right)\right)$ is regular, there is $x \in$ $\operatorname{GL}\left(V\left(\mathscr{F}_{\mathrm{cgt}}\right)\right)$ such that $x[A] \in \Omega_{\mathrm{cgt}}$. The key result in carrying over the preceding theory to the convergent setting is the following proposition. It leads to the theorem below.

Proposition. Let $A_{1}, A_{2}$ be regular elements of $\mathfrak{g l}\left(V\left(\mathscr{F}_{\text {cgt }}\right)\right)$. If $x \in$ $\mathrm{GL}(V(\mathscr{F}))$ and $x\left[A_{1}\right]=A_{2}$, then $x \in \mathrm{GL}\left(V\left(\mathscr{F}_{\text {cgt }}\right)\right)$.

Proof. We may assume that $A_{1}$ and $A_{2}$ are both in $\Omega_{\text {cgt }}$. We then have $x A_{1}+\dot{x}=A_{2} x$. Let $W=\operatorname{End}(V)$ and let $R$ be the element of $\mathfrak{g l}(W(\mathscr{F}))$ defined by $R u=A_{2} u-u A_{1}(u \in W(\mathcal{F}))$; then $x$, viewed as an element of $W(\mathscr{F})$, satisfies the differential equation $\dot{x}=R x$. The proposition is an immediate consequence of the following classical lemma.

LEMMA. Let $U$ be any finite dimensional vector space, $R \in \mathfrak{g l}(U(\mathcal{F})) a$ convergent connection of the first kind, and $x \in U(\mathcal{F})$ such that $\dot{x}=R x$. Then $x$ is convergent, with at least the same convergence disc as $R$. 
THEOREM. The convergent elements in any regular $\mathrm{GL}(V(\mathscr{F}))$-equivalence class form a single $\mathrm{GL}\left(V\left(\mathscr{F}_{\text {cgt }}\right)\right)$-equivalence class. If $A$ is a convergent regular connection, we can find $x \in \mathrm{GL}\left(V\left(\mathscr{F}_{\text {cgt }}\right)\right)$ such that $x[A]=z^{-1} C$ where $C \in \mathfrak{g l}(V)$ (resp. reduced $C \in \mathfrak{g l}(V)$ ). The map $[A] \rightarrow[\exp 2 \pi i C]$ (resp. $[A] \rightarrow[C]$ ) is then a well-defined bijection of the set of regular $\operatorname{GL}\left(V\left(\mathscr{F}_{\mathrm{cgt}}\right)\right)$-equivalence classes with the set of conjugacy classes (resp. reduced $\mathrm{GL}(V)$-orbits) in $\mathrm{GL}(V)$ (resp. $\mathrm{g} \mathfrak{l}(V)$ ).

\section{Transformations of connections with nilpotent leading coefficients.}

4.1. From now on our goal is to develop a reduction theory for a general connection of order $<-1$. The first step is to determine whether the connection is equivalent to one of higher order. It turns out that the key indicator for recognizing this is the nilpotence of the leading coefficient. It is easy to prove $(\$ 4.2)$ that if the leading coefficient of a connection is not nilpotent we cannot increase its order even if we are willing to use transformations with coefficients in algebraic extensions of $\mathscr{F}$. We shall see later $(\$ 6.2)$ that one can then split the connection "along" the spectral subspaces of its leading coefficient, and so one can continue the reduction by studying connections in lower dimension. Therefore the basic step in the reduction theory is to transform a connection into one whose leading coefficient is not nilpotent. The aim of this section is to develop the methods for doing this. It is quite easy to give examples showing that one cannot carry this step within $\mathscr{F}$ itself $(§ 4.2)$.

Our main result is the construction of a finitistic procedure to transform a connection $A$ of order $r<-1$ with nilpotent $A_{r}$, into a connection $A^{\prime}$ of order $r^{\prime} \geq r$ such that $A_{r^{\prime}}^{\prime}$ is either nonnilpotent, or, nilpotent but with $\operatorname{dim}\left(\mathrm{GL}(V) \cdot A_{r^{\prime}}^{\prime}\right)>\operatorname{dim}\left(\mathrm{GL}(V) \cdot A_{r}\right)$.

The basic idea behind it is to imbed the nilpotent leading coefficient $A_{r}$ of the connection $A$ in a standard triple $\left\{H, X, Y=A_{r}\right\}$ (§2.1) and transform $A$ via $\mathrm{GL}(V(\vartheta))_{1}$ to ensure that all the succeeding coefficients of $A$ are in the centralizer of $X$. We then obtain $A^{\prime}$ as the result of applying a transformation of the form $z^{q H}$ to $A, q$ being a rational number. We remark that this technique goes over without change when we consider connections with a reductive structure group. It is not surprising that the changes in the order of a connection are ultimately made only through the transformations $z^{q H}$. In fact, by the polar decomposition (cf. $\S 1.2$ ), $\operatorname{GL}\left(n, \mathscr{F}_{b}\right)=\operatorname{GL}\left(n, \mathcal{\theta}_{b}\right) \mathscr{D}_{+, b} \operatorname{GL}\left(n, \mathcal{\theta}_{b}\right)$, for any $b \geq 1$, where $\mathscr{D}_{+, b}=$ $\left\{z^{H} \mid H=\operatorname{diag}\left(r_{1}, \ldots, r_{n}\right), r_{j} \in(1 / b) \mathbf{Z}, r_{1} \leq r_{2} \leq \cdots \leq r_{n}\right\}$; and the order of elements of $\mathfrak{g l}(n, \overline{\mathscr{F}})$ is unchanged under $\operatorname{GL}(n, \bar{\theta})$.

The transformations $z^{q H}$ are essentially the so-called "shearing transformations" and occur already in Turrittin's work [26]. However, their 
relationship with $\mathfrak{g l}(2)$ and the idea of basing reduction theory on the orbit theory of nilpotents are both new.

4.2. Proposition. Let $A, A^{\prime} \in \mathfrak{g} \mathfrak{l}\left(V\left(\mathscr{F}_{b}\right)\right)$ and suppose that $A$ and $A^{\prime}$ are equivalent in $\mathrm{GL}\left(V\left(\mathscr{F}_{b}\right)\right)$, $b$ being an integer $\geq 1$. Let $r=\operatorname{ord}(A)$, $r^{\prime}=\operatorname{ord}\left(A^{\prime}\right)$. If $r<r^{\prime}$ and $r<-1$, then $A_{r}$ is a nilpotent element of $\mathrm{gl}(V)$.

Proof. Clearly (cf. §1.3) there is no loss of generality in assuming that $b=1$ since we may consider $z^{1 / b}$ as the independent variable. Let $A=x\left[A^{\prime}\right], x \in \mathrm{GL}(V(\mathscr{F}))$. We may assume $V=\mathbf{C}^{n}$. We write (cf. §1.2) $x=k_{1} d k_{2}$ where $k_{1}, k_{2} \in \mathrm{GL}(n, \theta)$ and $d=\operatorname{diag}\left(z^{a_{1}}, \ldots, z^{a_{n}}\right)$ where the $a$, are integers and $a_{1} \leq a_{2} \leq \cdots \leq a_{n}$. If $B=k_{1}^{-1}[A], B^{\prime}=k_{2}\left[A^{\prime}\right]$, then $B=d\left[B^{\prime}\right]$. On the other hand, if $k_{J}=x_{j 0}+z x_{j 1}+\cdots(j=1,2)$, it is clear that $B$ (resp. $B^{\prime}$ ) has the same order as $A$ (resp. $A^{\prime}$ ), and that $B_{r}=x_{10}^{-1} A_{r} x_{10}, B_{r^{\prime}}^{\prime}=x_{20} A_{r^{\prime}}^{\prime} x_{20}^{-1}$. So it is a question of proving that $B_{r}$ is nilpotent.

Let $B=\left(b_{\imath j}\right), B^{\prime}=\left(b_{i j}^{\prime}\right)$. Then

$$
b_{\imath j}(z)=b_{i j}^{\prime}(z) z^{a_{i}-a_{j}}+a_{i} \delta_{i j} / z \text {. }
$$

Suppose $i \geq j$. Then $a_{l} \geq a_{j}$ so that $b_{i j}^{\prime}(z) z^{a_{i}-a_{j}}$ has order $\geq r^{\prime}$. Hence the right side above has order $\geq \min \left(r^{\prime}, 1\right)>r$, i.e.,

$$
b_{\imath J} \equiv 0 \quad\left(\bmod z^{r+1}\right) \text {. }
$$

In other words, the leading coefficient matrix $B_{r}$ of $B$ has zeros on and below the main diagonal. It is therefore nilpotent.

For any $A \in \mathfrak{g} \mathfrak{l}(V(\mathscr{F}))$ we define its principal level pl( $A)$, by

$$
\operatorname{pl}(A)=\sup _{x \in \mathrm{GL}(V(\overline{\mathscr{F}}))} \min (-1, \text { ord } x[A]) .
$$

Thus

$$
\operatorname{pl}(A)= \begin{cases}-1 & \text { if ord } x[A] \geq-1 \\ \sup _{x \in \mathrm{GL}(V(\overline{\mathscr{F}}))}(\text { for some } x \in \mathrm{GL}(V(\overline{\mathscr{F}})) \\ & \text { if ord } x[A]<-1 \\ & \text { for all } x \in \mathrm{GL}(V(\overline{\mathscr{F}})) .\end{cases}
$$

This is obviously an invariant of the $\mathrm{GL}(V(\overline{\mathscr{F}}))$-equivalence class of $A$.

If $A \in \mathfrak{g l}(V(\overline{\mathscr{F}})), r=\operatorname{ord}(A)<-1$ and $A_{r}$ is not nilpotent, Proposition 4.2 shows that ord $x[A] \leq r$ for all $x \in \mathrm{GL}(V(\overline{\mathscr{F}}))$. Hence $p l(A)=r$. If $A_{r}$ is nilpotent it is not a priori clear that $\operatorname{pl}(A)$ is even a rational 
number. This is true and there is an $x \in \mathrm{GL}(V(\overline{\mathscr{F}}))$ such that $A^{\prime}=x[A]$ has order $r^{\prime}=p l(A)$ with $A_{r^{\prime}}^{\prime}$ not nilpotent.

In order to motivate our methods we shall look at an example. Let

$$
A=\left(\begin{array}{ll}
0 & 0 \\
1 & 0
\end{array}\right) z^{-2}+\left(\begin{array}{ll}
0 & 1 \\
0 & 0
\end{array}\right) z^{-1} .
$$

Let us fix a square root $z^{1 / 2}$ and consider $A^{\prime}=x[A]$ where $x=$ $\operatorname{diag}\left(1, z^{1 / 2}\right)$. Then

$$
A^{\prime}=\left(\begin{array}{ll}
0 & 1 \\
1 & 0
\end{array}\right) z^{-3 / 2}+\left(\begin{array}{cc}
0 & 0 \\
0 & 1 / 2
\end{array}\right) z^{-1}
$$

The leading coefficient of $A^{\prime}$ is $\left(\begin{array}{ll}0 & 1 \\ 1 & 0\end{array}\right)$ which is not nilpotent. Hence $p l(A)=p l\left(A^{\prime}\right)=-3 / 2$. In particular, we cannot increase the order of $A$ using GL $(V(\mathcal{F}))$; for, if we could do so, $A$ would be regular and $A^{\prime}$ would be equivalent over $\operatorname{GL}(V(\overline{\mathscr{F}}))$ to an element of the form $z^{-1} C$, contradicting the above Proposition.

In the notation of $\$ 2.1$ (cf. (2.1a)) $A$ can be written as $Y z^{-2}+X z^{-1}$. The simplicity of the step going from $A$ to $A^{\prime}$ is clearly due to the fact that $X$ has very good transformation properties relative to the "shearing transformation" $\operatorname{diag}\left(1, z^{1 / 2}\right)$. This can be further illustrated with the example of a connection $A$ in $\mathfrak{g l}(n, \mathscr{F})$ of order $r<-1$ whose leading coefficient $A_{r}$ is the principal nilpotent $Y_{n}$ defined by (2.13) and (2.14). The opposite nilpotent is then $X_{n}$ defined by (2.15) and its centralizer is the space of companion matrices. If we consider the action of $x=$ $\operatorname{diag}\left(z^{q}, z^{2 q}, \ldots, z^{n q}\right)$ we find that

$$
x\left[Y_{n} z^{r}\right]=Y_{n} z^{r+q}+\operatorname{diag}(q, 2 q, \ldots, n q) z^{-1}
$$

while

$$
x\left[Z_{l} z^{r+m}\right]=Z_{i} z^{r+m-q(l-1)}+\operatorname{diag}(q, 2 q, \ldots, n q) z^{-1} .
$$

This suggests that if we can arrange matters so that all the coefficients $A_{r+m}(m \geq 1)$ are in the centralizer of $X_{n}$, then one can, by suitable choice of $q$, match the numbers $r+q$ with the numbers $r+m-q(i-1)$. The result will be to obtain a connection $A^{\prime}$ whose leading coefficient will be of the form $Y_{n}+c_{1} Z_{1}+\cdots+c_{n} Z_{n}$ where not all $c_{i}$ are 0 , hence nonnilpotent by Proposition 2.2.2. In the example considered earlier, we have $q=1 / 2$ and the new leading coefficient is $Y+X$. This type of argument is completely general and is the core of our method.

The first step is therefore to try to transform $A$ so that all the $A_{r+m}$ ( $m \geq 1$ ) are centralized by a nilpotent opposite to $A_{r}$. Now, we know from $\S 2.1$ that if $\{H, X, Y\}$ is a standard triple, the centralizer of $X$ is a 
subspace of $\mathfrak{g} \mathfrak{l}(V)$ complementary to $\operatorname{range}(\operatorname{ad} Y)$. So we begin the next paragraph with the setting in which $\mathfrak{a}$ is an arbitrary subspace of $\mathfrak{g l}(V)$ complementary to the range of $\operatorname{ad}\left(A_{r}\right)$.

\subsection{Proposition. Let}

$$
A=z^{r} A_{r}+z^{r+1} A_{r+1}+\cdots \quad(r<-1)
$$

be an element of $\mathfrak{g l}(V(\mathcal{F}))$. Let $\mathfrak{a} \subset \mathfrak{g} \mathfrak{l}(V)$ be a linear subspace complementary to $\operatorname{range}\left(\operatorname{ad}\left(A_{r}\right)\right)$. Then we can find $x \in \mathrm{GL}(V(\theta))_{1}$ such that if $A^{\prime}=x[A]$, then, writing

$$
A^{\prime}=z^{r} A_{r}^{\prime}+z^{r+1} A_{r+1}^{\prime}+\cdots
$$

we have

(a) $A_{r}^{\prime}=A_{r}$

(b) $A_{r+m}^{\prime} \in$ a $(m \geq 1)$.

Proof. We define a sequence of elements

$$
A=A^{(0)}, A^{(1)}, A^{(2)}, \ldots
$$

as follows. We define $A^{(1)}$ by

$$
A^{(1)}=x^{(1)}[A], \quad x^{(1)}=1+z T_{1}
$$

where $T_{1}$ will be chosen so that $A_{r+1}^{(1)} \in$ a. The condition on $A_{r+1}^{(1)}$ turns out to be

$$
A_{r+1}^{(1)}=A_{r+1}-\operatorname{ad}\left(A_{r}\right)\left(T_{1}\right) .
$$

From the definition of $a$ it is obvious that we can choose $T_{1} \in \mathfrak{g l}(V)$ so that $A_{r+1}-\operatorname{ad}\left(A_{r}\right)\left(T_{1}\right) \in$ a.

Assume now that $A^{(1)}, \ldots, A^{(m)}$ are chosen with

$$
A^{(k)}=x^{(k)}\left[A^{(k-1)}\right], \quad x^{(k)}=1+z^{k} T_{k} \quad(1 \leq k \leq m)
$$

such that the coefficients of $A^{(k)}$ satisfy

$$
A_{r+p}^{(k)} \in \mathfrak{a}, \quad p=1,2, \ldots, k .
$$

We take

$$
A^{(m+1)}=x^{(m+1)}\left[A^{(m)}\right], \quad x^{(m+1)}=1+z^{m+1} T_{m+1} .
$$

Then $A_{r}^{(m+1)}=A_{r}, A_{r+p}^{(m+1)}=A_{r+p}^{(m)}(1 \leq p \leq m)$, and

$$
A_{r+m+1}^{(m+1)}=A_{r+m+1}^{(m)}-\operatorname{ad}\left(A_{r}\right)\left(T_{m+1}\right) .
$$


We choose $T_{m+1}$ so that $\operatorname{ad}\left(A_{r}\right)\left(T_{m+1}\right)$ equals the projection of $A_{r+m+1}^{(m)}$ on $\operatorname{range} \operatorname{ad}\left(A_{r}\right)(\bmod a) ;$ then $A_{r+m+1}^{(m+1)} \in \mathfrak{a}$. The definition of $x^{(m+1)}$ and $A^{(m+1)}$ with the required properties is now complete.

If $x \in \mathrm{GL}(V(\theta))_{1}$ is defined by

$$
x=\prod_{1}^{\infty}\left(1+z^{m} T_{m}\right)=\lim _{m \rightarrow \infty}\left(1+z^{m} T_{m}\right) \cdots\left(1+z T_{1}\right)
$$

and $A^{\prime}=x[A]$, then $x$ and $A^{\prime}$ have the required properties.

REMARK. In the above construction the only lack of uniqueness is in choosing the $T_{m+1}$; let $\mathfrak{b} \subset \mathfrak{S} \mathfrak{l}(V)$ be a subspace such that

$$
\mathfrak{g} \mathfrak{l}(V)=N\left(\operatorname{ad} A_{r}\right) \oplus \mathfrak{b}
$$

where $N\left(\operatorname{ad} A_{r}\right)$ is the null space of ad $A_{r}$. Then

$$
\operatorname{ad}\left(A_{r}\right): \mathfrak{b} \stackrel{\sim}{\rightarrow} \operatorname{range}\left(\operatorname{ad} A_{r}\right)
$$

is an isomorphism. It is now clear that once $\mathfrak{a}$ and $\mathfrak{b}$ are given the entire process is uniquely determined. We have thus obtained the following corollary.

Corollary. Fix a and $\mathfrak{b}$ as above. Then there exists a unique $x=x_{A}$ $\in \mathrm{GL}(V(\theta))_{1}$ of the form (4.8) with $T_{m+1} \in \mathfrak{b}$ for all $m \geq 0$, such that, if $A^{\prime}=x[A], A_{r}^{\prime}=A_{r}$ and $A_{r+m}^{\prime} \in \mathfrak{U}(m \geq 1)$.

4.4. Fix $a$ and $\mathfrak{b}$ as in $\S 4.3$. It is obvious that $T_{1}$ depends only on $A_{r}$ and $A_{r+1}$. For general $m$, we have

$$
\begin{aligned}
A_{r+t}^{(m+1)}= & A_{r+t}^{(m)}+T_{m+1} A_{r+t-m-1}^{(m)} \\
& -A_{r+t-m-1}^{(m+1)} T_{m+1}+(m+1) \delta_{r+t, m} T_{m+1} \quad(t \geq 1)
\end{aligned}
$$

with the convention that $A_{s}=0$ for $s<r$. A simple induction then yields the following proposition and its corollary.

Proposition. For any $m \geq 0, T_{m+1}$ depends only on $A_{s}(r \leq s \leq r+m$ $+1)$. Moreover, $A_{r+m}^{\prime}$ depends only on $A_{s}(r \leq s \leq r+m)$.

Corollary. Let $M$ be any integer $\geq 1$ and $A$ any connection of order $r<-1$. Let $\Omega(A, M)$ be the set of all connections $B$ such that $B$ has order $r$ and $B_{r+s}=A_{r+s}$ for $0 \leq s<M$. If the element $x_{A}$ is as in Corollary 4.3 and if

$$
x_{A, M}=y=\left(1+z^{M-1} T_{M-1}\right) \cdots\left(1+z T_{1}\right)
$$


then all the connections $B^{\prime}=y[B], B \in \Omega(A, M)$, have the same coefficients $B_{r+s}^{\prime}, 0 \leq s<M$; with $B_{r}^{\prime}=A_{r}$ and $B_{r+s}^{\prime} \in \mathfrak{a}, 1 \leq s<M$. Moreover, for each $B \in \Omega(A, M), x_{B}$ has the product representation

$$
x_{B}=\prod_{r=1}^{\infty}\left(1+z^{r} T_{r}^{\prime}\right)
$$

where $T_{r}^{\prime}=T_{r}$ for $1 \leq r<M$.

4.5. We can now begin the reduction of a connection $A$ of order $r<1$ with nilpotent leading coefficient $A_{r}$. We need some preparation. We choose a standard triple $\{H, X, Y\}$ with $A_{r}=Y$, and take $\mathfrak{a}=\mathfrak{g}_{X}$, $\mathfrak{b}=\operatorname{range}(\operatorname{ad} X)$ in the discussion of $\S \S 4.3-4.4$.

Let $\Lambda=\Lambda(Y)$ be as in (2.5). We assume that

(i) $A_{r}=Y$

(ii) $A_{r+m} \in \mathrm{g}_{X}, \quad 1 \leq m<\Lambda(|r|-1)$.

Let $\left(Z_{l}\right)_{1 \leq l \leq q}$ be as in (2.4). Then we can write

$$
A_{r+m}=\sum_{1 \leq k \leq q} a_{r+m, k} Z_{k} \quad(1 \leq m<\Lambda(|r|-1)) .
$$

Although it would seem more natural (in view of $\S 4.3$ ) to assume (ii) of (4.9) for all $m \geq 1$, we work with the weaker assumption (4.9); this will make possible the construction of algorithms later on. Let $\delta=\delta(A)$ be defined by

$$
\begin{array}{r}
\delta=\inf \left\{m /\left(\frac{1}{2} \lambda_{k}+1\right) \mid 1 \leq m<\Lambda(|r|-1),\right. \\
\left.1 \leq k \leq q, a_{r+m, k} \neq 0\right\} .
\end{array}
$$

Note that $\delta$ depends on $A$, but only through its coefficients $A_{r+m}$ with $m<\Lambda(|r|-1)$. We set $\delta=\infty$ if $A_{r+m}=0$ for $1 \leq m<\Lambda(|r|-1)$.

For any semisimple $H \in \mathfrak{g l}(V)$ with only integral eigenvalues and any $m \in(1 / b) \mathbf{Z}(b \geq 1$ an integer) we have defined in $\S 1.5$ the element $z^{m H} \in \mathrm{GL}\left(V\left(\mathscr{F}_{b}\right)\right)$; it depends on the choice of $z^{1 / b}$ and $m \mapsto z^{m H}$ is a homomorphism of the additive group of $(1 / b) \mathbf{Z}$ into $\operatorname{GL}\left(V\left(\mathscr{F}_{b}\right)\right)$ (even $\left.\mathrm{GL}\left(V\left(\mathscr{F}_{b, \mathrm{cgt}}\right)\right)\right)$. If $M \in \mathfrak{g l}(V)$ is such that $[H, M]=c M$ for some $c \in \mathbf{Z}$, a simple calculation shows that

$$
z^{m H} M z^{-m H}=z^{c m} M \quad(m \in(1 / b) \mathbf{Z}) .
$$

4.6. The following result is the key step in our reduction theory. 
Proposition. Let notation be as above.

(a) If $|r|-1 \leq \delta \leq \infty$, then, for

$$
x=z^{-(|r|-1) H / 2} \in \mathrm{GL}\left(V\left(\mathscr{F}_{2}\right)\right) \text {, }
$$

$x[A]=A^{\prime}$ is of the first kind, $\in \mathfrak{g} \mathfrak{I}\left(V\left(\mathscr{F}_{2}\right)\right)$ and $A_{-1}^{\prime}$ depends only on the $A_{r+m}, 0 \leq m \leq \Lambda(|r|-1)$.

(b) If $0<\delta<|r|-1$, then $\delta=a / b$ where $a, b$ are integers $\geq 1$ with $1 \leq b \leq \Lambda$. Choose such a representation of $\delta$ and make a choice of $z^{1 / 2 b}$ in $\mathscr{F}_{2 b}$. Let

$$
x=z^{-\delta H / 2}, \quad A^{\prime}=x[A] .
$$

Then $x \in \mathrm{GL}\left(V\left(\mathscr{F}_{2 b}\right)\right), A^{\prime} \in \mathfrak{g l}\left(V\left(\mathscr{F}_{2 b}\right)\right), A^{\prime}$ has order $r+\delta<-1$; and if we write $m_{k}=\left(\frac{1}{2} \lambda_{k}+1\right) \delta$ for any $k$, we have $m_{k}<\Lambda(|r|-1)$ and

$$
A_{r+\delta}^{\prime}=Y+\sum_{1 \leq k \leq q, m_{k} \in \mathbf{Z}} a_{r+m_{k}, k} Z_{k} \neq Y \text {. }
$$

In particular, $A_{r+\delta}^{\prime}$ is determined by the $A_{r+s}, 0 \leq s<\Lambda(|r|-1)$; moreover, either $A_{r+\delta}^{\prime}$ is not nilpotent, in which case $p l(A)=r+\delta$, or, nilpotent but belongs to an orbit of dimension greater than $\operatorname{dim}(\mathrm{GL}(V) \cdot Y)$.

Proof. From the definition it is obvious that when $0<\delta<|r|-1, \delta$ has a representation of the form described. In this case, for some $k, m$ with $1 \leq k \leq q, \quad 1 \leq m<\Lambda(|r|-1)$, we have $a_{r+m, k} \neq 0$ and $\delta=$ $m /\left(1+\frac{1}{2} \lambda_{k}\right)$. Then $m=m_{k} \in \mathbf{Z}$, and the element in the right side of (4.13) is different from $Y$, i.e., $A_{r+\delta}^{\prime} \neq Y$. Proposition 2.2 .2 now implies that last statement.

It is convenient to prove (a) and (b) together. Write

$$
\delta^{*}=\min (|r|-1, \delta), \quad x=z^{-\delta^{*} H / 2}, \quad M=\Lambda(|r|-1),
$$

and let $A^{\prime}=x[A]$. We enlarge the basis $Z_{\imath}(1 \leq i \leq q)$ to a basis $Z_{j}$ $(1 \leq j \leq p)$ of $\mathfrak{g}=\mathfrak{g l}(V)$ such that $\left[H, Z_{j}\right]=h_{j} Z_{j}, 1 \leq j \leq p$. Since the eigenvalues of $H$ in the irreducible module for $\mathbf{C} \cdot H+\mathbf{C} \cdot X+\mathbf{C} \cdot Y$ with highest weight $\lambda$ are integers in $[-\lambda, \lambda]$, we have $\left|h_{j}\right| \leq \max _{1 \leq l \leq q}\left(\lambda_{l}\right)$. Then $A$ can be written in the form

$$
\begin{aligned}
A= & Y z^{r}+\sum_{1 \leq m<M} z^{r+m} \sum_{1 \leq k \leq q} a_{r+m, k} Z_{k} \\
& +\sum_{m \geq M} z^{r+m} \sum_{1 \leq j \leq p} b_{r+m, j} Z_{j}
\end{aligned}
$$


so that, by (4.12),

$$
\begin{aligned}
A^{\prime}= & Y z^{r+\delta^{*}}+\sum_{1 \leq m<M} z^{r+m} \sum_{1 \leq k \leq q} z^{-\lambda_{k} \delta^{*} / 2} a_{r+m, k} Z_{k} \\
& +\sum_{m \geq M} z^{r+m} \sum_{1 \leq j \leq p} z^{-h, \delta^{*} / 2} b_{r+m, J} Z_{J}-\frac{1}{2} \delta^{*} z^{-1} H .
\end{aligned}
$$

We shall now prove that the sums above contain powers $z^{k}$ only for $k \geq r+\delta^{*}$ and that the coefficient of $z^{r+\delta^{*}}$ comes only from the terms with $m<M$ if $0<\delta<|r|-1$, and from the terms with $m \leq M$ if $|r|-1 \leq \delta \leq \infty$. First let $m \geq M$. Since $\left|h_{J}\right| \leq \max _{1 \leq i \leq q}\left(\lambda_{l}\right)$, we have

$$
\begin{aligned}
r+m-\frac{1}{2} h_{\jmath} \delta^{*} & \geq r+m-\delta^{*}(\Lambda-1) \\
& =r+(m-M)+\Lambda\left(|r|-1-\delta^{*}\right)+\delta^{*} ;
\end{aligned}
$$

this is $>r+\delta$ if $0<\delta<|r|-1$ (so that $\delta^{*}=\delta$ ), $\geq r+\delta^{*}$ always, and $>r+\delta^{*}$ unless $m=M, \delta^{*}=|r|-1$ (i.e., $|r|-1 \leq \delta \leq \infty$ ). Suppose $1 \leq m<M, a_{r+m, k} \neq 0$ for some $k, 1 \leq k \leq q$. For any such $m, k$, $0<\delta \leq m /\left(1+\frac{1}{2} \lambda_{k}\right)$ so that $r+m-\frac{1}{2} \lambda_{k} \delta^{*} \geq r+\delta\left(1+\frac{1}{2} \lambda_{k}\right)$ $-\frac{1}{2} \delta^{*} \lambda_{h} \geq r+\delta+\frac{1}{2} \lambda_{k}\left(\delta-\delta^{*}\right) \geq r+\delta$. We have therefore proved (a) and (b) except for the explicit expression (4.13) for $A_{r+\delta}^{\prime}$ when $0<\delta<|r|$ -1 . But it is clear from the above discussion that the term $z^{r+\delta}$ comes from $m, k$ with $1 \leq m<M, 1 \leq k \leq q, a_{r+m, k} \neq 0$ and $m-\frac{1}{2} \lambda_{k} \delta=\delta$, yielding (4.13). Since $\delta<|r|-1$ and $1+\frac{1}{2} \lambda_{k} \leq \Lambda$, we have $m_{k}=$ $\delta\left(1+\frac{1}{2} \lambda_{k}\right)<\Lambda(|r|-1)$. This proves the proposition.

COROLlaRy 1. Let in addition $A_{r}$ be principal and assume (as we may) that $A_{r}=Y_{n}$ as in (2.13) and (2.14). Then $\Lambda=n$ and

$$
\delta=\inf \left\{\frac{m}{j-i+1} \mid 1 \leq m<n(|r|-1), i \leq j,\left(A_{r+m}\right)_{i,} \neq 0\right\} .
$$

Furthermore, in case (a) we can take

$$
x=\operatorname{diag}\left(z^{|r|-1}, z^{2(|r|-1)}, \ldots, z^{n(|r|-1)}\right) \in \mathrm{GL}(n, \mathscr{F})
$$

while in case (b) we can take

$$
x=\operatorname{diag}\left(z^{a / b}, z^{2 a / b}, \ldots, z^{n a / b}\right) \in \operatorname{GL}\left(n, \mathscr{F}_{b}\right) .
$$

Proof. To get $\delta$ in the stated form we note that (cf. (2.16)-(2.18)) $\left(A_{r+m}\right)_{i j}=a_{r+m, j-i}$ and $\lambda_{j-i}=2(j-i)$. Further in case (a) we observe that $z^{-(1 / 2)(|r|-1) H}$ equals

$$
z^{-(|r|-1)(n+1) / 2} \operatorname{diag}\left(z^{|r|-1}, \ldots, z^{n(|r|-1)}\right)
$$


and note that omitting the power of $z$ in front does not change the conclusion.

In case (b) the argument is the same, the power of $z$ which comes out in front being $z^{-(1 / 2) \delta(n+1)}$. Omission of this power changes only the coefficient of $z^{-1}$ which is not involved in the conclusion. Finally since we are working with nilpotents of maximal dimension there is only one possibility for $A_{r+\delta}^{\prime}$, namely, it is not nilpotent.

Corollary 2. Suppose $\operatorname{tr}(A)=0$, i.e., all the coefficients of $A$ are in $\mathfrak{g}(V)$. Then, $A^{\prime}$ has the same property. In this case, if $0<\delta<|r|-1$ and $A_{r+\delta}^{\prime}$ is not nilpotent, it must have at least two distinct eigenvalues.

Proof. Since $H \in g \mathfrak{l}(V)$, the formula $A^{\prime}=x A x^{-1}+(\alpha / z) H$ where $\alpha \in \mathbf{C}$ shows that $\operatorname{tr}\left(A^{\prime}\right)=0$. The Corollary is immediate.

Let $M$ be an integer $\geq 1$. For $B^{(1)}, B^{(2)} \in \mathfrak{g} \backslash(V(\mathscr{F}))$, both of order $r$, we write $B^{(1)} \equiv B^{(2)}(M)$ if $B_{r+s}^{(1)}=B_{r+s}^{(2)}$ for $0 \leq s<M$. We then have the following useful supplement to (b) of the above Proposition.

Corollary 3. Let $0<\delta<r-1, \delta=a / b$ where $a, b$ are integers $\geq 1$. Let $B \in \mathfrak{g} l(V(\mathscr{F}))$ be of order $r$ and $A \equiv B(M)$ where $M \geq \Lambda(|r|-1)$. Let $x=z^{-(1 / 2) \delta H}, A^{\prime}=x[A], B^{\prime}=x[B]$. Then $B^{\prime}$ is of order $r+\delta<-1$, $A_{r+\delta}^{\prime}=B_{r+\delta}^{\prime}$, and the $\zeta$-liftings $\left(\zeta=z^{1 / 2 h}\right) \tilde{A}^{\prime}$ of $A^{\prime}$ and $\tilde{B}^{\prime}$ of $B^{\prime}$ satisfy

$$
\tilde{A}^{\prime} \equiv \tilde{B}^{\prime}(2 b M-2 a \Lambda) \text {. }
$$

Proof. Only the last statement requires proof. We have from (4.12) the following consequence: if $H^{\prime}$ is any semisimple endomorphism of $V$ with integral eigenvalues, then, for any $m^{\prime} \in(1 / 2 b) \mathbf{Z}$, the lag of $z^{m^{\prime} H^{\prime}}$ will be $\leq\left|m^{\prime}\right| \max |h|$ where the maximum is over the eigenvalues $h$ of ad $H^{\prime}$; indeed, this is most easy to see by writing the coefficients of any connection as a linear combination of eigenvectors of ad $H^{\prime}$ and using (4.12) with respect to each term in this linear combination. If $H^{\prime}$ is $H$ and $m^{\prime}=-\frac{1}{2} \delta, \max |h|=2(\Lambda-1)$ so that the lag is $\leq(\Lambda-1) \delta$. Hence, with $x=z^{-(1 / 2) \delta H}$,

$$
\begin{aligned}
A \equiv B \quad\left(\bmod z^{r+M}\right) & \Rightarrow A^{\prime} \equiv B^{\prime} \quad\left(\bmod z^{r+M-\delta(\Lambda-1)}\right) \\
& \Rightarrow \tilde{A}^{\prime} \equiv \tilde{B}^{\prime} \quad\left(\bmod \zeta^{N}\right)
\end{aligned}
$$

where $N=2 b(r+M-\delta(\Lambda-1))+2 b-1=(2 b r+2 a+2 b-1)+$ $2 b M-2 a \Lambda=\tilde{r}+2 b M-2 a \Lambda$. Hence $\tilde{A}^{\prime} \equiv \tilde{B}^{\prime}(2 b M-2 a \Lambda)$. 


\section{The principal level.}

5.1. In this section we shall illustrate the technique of $\$ 4.6$ by applying it to determine the basic properties of the principal level of a connection. The present section is therefore a prelude to the full reduction theory over $\overline{\mathcal{F}}$.

The main properties of the principal level are as follows: (i) it is a rational number $\in m^{-1} \mathbf{Z}$ where $1 \leq m \leq n=\operatorname{dim}(V)$, and is -1 if and only if the connection is regular (ii) $p l(A)$ is already determined by the coefficients $A_{r+s}, 0 \leq s<n(|r|-1), r$ being the order of $A$; moreover, this estimate is sharp (iii) there is an $x \in \mathrm{GL}\left(V\left(\overline{\mathscr{F}}_{\text {cgt }}\right)\right)$ such that $\operatorname{ord}(x[A])$ $=p l(A)$.

We shall use the results of $\$ 4.6$ to prove these properties and to develop algorithms for computing $p l(A)$. In the regular case we shall supplement these procedures with a technique for calculating the monodromy of the given connection. Procedures for deciding when $A$ is regular have been developed by Moser [19] and Lutz [17]. The estimate on the number of coefficients needed to determine $p l(A)$ is known (cf. [12]). Our techniques, besides being purely algebraic, will lead in $\S 6$ to the much stronger result that the coefficients $A_{r+s}, 0 \leq s<n(|r|-1)$, are already sufficient to determine the entire irregular part of the canonical form of $A$.

5.2. The following is the basic result on the principal level.

THEOREM. Let $A \in \mathfrak{g l}(V(\mathscr{F}))$. Then:

(a) $A$ is regular if and only if $p l(A)=-1$.

(b) If $A$ is not regular and $r^{\prime}=p l(A)$, then $r^{\prime}$ is a rational number $<-1$ and belongs to $m^{-1} \mathbf{Z}$ where $1 \leq m \leq n$; and we can find $x \in$ $\mathrm{GL}\left(V\left(\widetilde{\mathscr{F}}_{\mathrm{cgt}}\right)\right)$ such that $A^{\prime}=x[A]$ has order $r^{\prime}$, and $A_{r^{\prime}}^{\prime}$ is not nilpotent.

Proof. We prove (a) and (b) together except for the assertion that $r^{\prime} \in m^{-1} \mathbf{Z}$ where $1 \leq m \leq n$; this fact will be obtained in Proposition 7.6. If $\operatorname{ord}(A)=r$ is $\geq-1, A$ is regular and $p l(A)=-1$. So we may assume $r<-1$. If $\operatorname{dim}(V)=1, A_{r}$ is a nonzero complex number and so, $p l(A)=r$ and we are in case (b) (with $x=1$ ). So we may assume $\operatorname{dim}(V) \geq 2$.

Case 1. $A_{r}$ is not nilpotent. Then $p l(A)=r$ and we are in the context of (b) with $x=1$.

Case 2. $A_{r}$ is nilpotent. We use downward induction on $\operatorname{dim}\left(\mathrm{GL}(V) \cdot A_{r}\right)$. Let us write $Y=A_{r}$ and fix $H, X \in \mathfrak{g} \mathfrak{l}(V)$ such that 
$\{H, X, Y\}$ is a standard triple. We may clearly assume that $A_{r+m} \in g_{X}$ for all $m \geq 1$ (see §4.3). Proposition 4.6 gives an element $x \in \mathrm{GL}\left(V\left(\widetilde{\mathscr{F}}_{\mathrm{cgt}}\right)\right)$ such that $A^{\prime}=x[A]$ is in one of three categories: first kind, of order $r^{\prime}<-1$ with $A_{r^{\prime}}^{\prime}$ non-nilpotent, or with $A_{r^{\prime}}^{\prime}$ nilpotent but with $\operatorname{dim}\left(\mathrm{GL}(V) \cdot A_{r^{\prime}}^{\prime}\right)>\operatorname{dim}\left(\mathrm{GL}(V) \cdot A_{r}\right)$. In the first two cases we are through trivially; in the third case we are through by the induction hypothesis. We must note that $A^{\prime}$ may not be in $\mathfrak{g l}(V(\mathscr{F}))$ so that it may be necessary to first change the independent variable to $\zeta=z^{1 / b}$.

If the connection $A$ arises from a scalar differential equation it is of the form

$$
A=\left(\begin{array}{ccccc}
0 & 1 & & 0 & \\
& 0 & & & \\
\vdots & & \ddots & 1 & \\
-a_{n}, & -a_{n-1}, & \cdots & -a_{2}, & -a_{1}
\end{array}\right)
$$

with $a_{i} \in \mathcal{F}$. In this case one can calculate $\operatorname{pl}(A)$ directly in terms of the coefficients $a_{i}$. In fact we have the formula

$$
p l(A)=\min \left\{-1, \frac{\operatorname{ord}\left(a_{1}\right)}{1}, \frac{\operatorname{ord}\left(a_{2}\right)}{2}, \ldots, \frac{\operatorname{ord}\left(a_{n}\right)}{n}\right\}
$$

due essentially to Katz (cf. [13], §11), which generalizes the classical result of Fuchs that $A$ is regular if and only if $\operatorname{ord}\left(a_{j}\right) \geq-j$ for $1 \leq j \leq n$. We sketch a proof of this from our point of view which is a variant of Proposition 4.6. Let $\left\{H_{n}, X_{n}, Y_{n}\right\}$ be the standard triple in $\mathfrak{g} \mathfrak{l}(n, \mathbf{C})$ defined by (2.13)-(2.15).

Proposition 2. Let $A$ be as in (5.1) and let $s$ be defined by the right side of (5.2). If $x=z^{(s / 2) H_{n}}$ and $A^{\prime}=x[A]$, then $A^{\prime}$ is of the first kind if $s=-1$ while $\operatorname{ord}\left(A^{\prime}\right)=s$ and $A_{s}^{\prime}$ is non-nilpotent if $s<-1$. In particular, $s=$ $\operatorname{pl}(A)$.

Proof. A simple calculation shows that $A^{\prime}=A^{\prime \prime}+(s / 2) H_{n} z^{-1}$ where

$$
A^{\prime \prime}=\left(\begin{array}{ccccc}
0 & z^{s} & & \\
0 & \ddots & 0 & \\
0 & & \ddots & z^{s} & \\
& & & 0 & z^{s} \\
-a_{n} z^{-(n-1) s}, & -a_{n-1} z^{-(n-2) s} & , \ldots, & -a_{2} z^{-s}, & -a_{1}
\end{array}\right)
$$


By the definition of $s$, ord $\left(a_{j}\right)-(j-1) s \geq s$, so that $\operatorname{ord}\left(A^{\prime \prime}\right) \geq s$. If $s=-1$, we are done. If $s<-1$, then $s$ is the minimum of the numbers $\operatorname{ord}\left(a_{j}\right) / j, 1 \leq j \leq n$, so that some entry in the last row of $A^{\prime \prime}$ has order exactly $s$. So $A_{s}^{\prime}$ is the matrix given by

$$
A_{s}^{\prime}=\left(\begin{array}{ccccc}
0 & 1 & & & \\
& 0 & & & \\
0 & & \ddots & & \\
-\alpha_{n}, & \ldots & & 0 & 1 \\
-\alpha_{2}, & -\alpha_{1}
\end{array} \mid\right.
$$

where at least one of the $\alpha_{i}$ is non-zero. $A_{s}^{\prime}$ is not nilpotent since its characteristic polynomial is

$$
\lambda^{n}+\alpha_{1} \lambda^{n-1}+\cdots+\alpha_{n} \neq \lambda^{n}
$$

REMARK. Let us consider the differential equation

$$
P_{n}(w) \frac{d^{n} y}{d w^{n}}+P_{n-1}(w) \frac{d^{n-1} y}{d w^{n-1}}+\cdots+P_{0}(w) y=0
$$

where $P_{i}$ are polynomials in $w, \operatorname{deg}\left(P_{i}\right)=M_{i}$, locally around $w=\infty$. If we go over to the corresponding system and let $z=1 / w$, we obtain a system $\dot{u}=A u$. The above proposition then gives

$$
p l(A)=\min (-1,-2-h)
$$

where

$$
h=\max _{i}\left(\frac{M_{i}-M_{n}}{n-i}\right)
$$

is the rank of $(*)$ according to [20].

5.3. From the (adic-)continuity properties of the action of $\operatorname{GL}(V(\overline{\mathscr{F}}))$ on $g \mathfrak{l}(V(\overline{\mathscr{F}}))$ it follows that the principal level, regarded as a function on $\mathfrak{g} \mathfrak{l}(V(\mathscr{F}))$, is locally constant. One can do much better however; the results of $\$ 4.6$ may be used to prove that $p l(A)$ can already be determined from the knowledge of the coefficients $A_{r+m}, 0 \leq m<n(|r|-1)$.

THEOREM. Let $r$ be any integer $<-1$, let $M=n(|r|-1)$, and let $\Omega_{r}$ be the set of all $A \in \mathrm{gl}(V(\mathscr{F}))$ of order $r$. If $A, B \in \Omega_{r}$ and $A_{r+m}=B_{r+m}$ for $0 \leq m<M$, then $\operatorname{pl}(A)=\operatorname{pl}(B)$. Moreover, this estimate is sharp. 
Proof. We shall obtain the theorem as a consequence of the following somewhat stronger proposition. For $A \in \Omega_{r}$, and any integer $L \geq 1$, $\Omega(A, L)$ is the set of $B \in \Omega_{r}$ with $A \equiv B(L)$ (cf. §4.6).

Proposition. Let $A \in \Omega_{r}$. Then we can find $u \in{ }^{\circ} \mathrm{GL}\left(V\left(\overline{\bar{F}_{\text {cgt }}}\right)\right)(c f$. \$1.7) such that

(i) the lag of $u \leq(n-1)(|r|-|s|)$ where $s=p l(A)$;

(ii) if $A^{*}=u[A], \operatorname{ord}\left(A^{*}\right) \geq s$ (with equality if $\left.s<-1\right)$.

In particular, if $s<-1, A_{s}^{*}$ is not nilpotent; and, for any $B \in \Omega(A, M)$, $B^{*}=u[B]$ has also order $s$, with $B_{s}^{*}=A_{s}^{*}$. If $s=-1$, then $B^{*}$ is of the first kind for any $B \in \Omega(A, M)$; if moreover $B_{r+M}=A_{r+M}$, then $A_{-1}^{*}=B_{-1}^{*}$ also.

Proof. It will be clear from the following proof that $u$ will be convergent. Before beginning the proof we remark that the assertions involving $B$ follow from (i) and (ii). Indeed, if $B \in \Omega(A, M), B \equiv A$ $\left(\bmod z^{r+M}\right)$ so that, as $M-\operatorname{lag}(u) \geq n(|r|-1)-(n-1)(|r|-1) \geq|r|$ $-1, B^{*} \equiv A^{*}\left(\bmod z^{-1}\right) ;$ if $B \equiv A\left(\bmod z^{r+M+1}\right), r+M+1-\operatorname{lag}(u) \geq$ $r+|r|=0$ so that we get the sharper result that $B^{*}-A^{*}$ has order $\geq 0$. The statements concerning $B$ are now clear. If $A_{r}$ is not nilpotent, we are through with $s=r, u=1$. So we may assume that $A_{r}$ is nilpotent. We prove the proposition by downward induction on the dimension of the orbit of $A_{r}$. Let $\{H, X, Y\}$ be a standard triple with $Y=A_{r}$. By Corollary 4.4 (with $\mathfrak{a}=\mathfrak{g}_{X}, \mathfrak{b}=\operatorname{range}$ ad $X$ ) we know that if $y=x_{A, M}$ as in that Corollary and $A^{\prime}=y[A]$, then $A_{r+s}^{\prime} \in \mathfrak{g}_{X}, 0 \leq s<M$. Proposition 4.6 and its Corollaries now lead to the following conclusions. For $|r|-1 \leq \delta$ $\leq \infty$, if we take $x=z^{-(1 / 2)(|r|-1) H}, u=x y$, then $A^{*}=u[A]=x\left[A^{\prime}\right]$ is of the first kind; thus $s=-1$. Further (cf. proof of Corollary 4.6.3), $\operatorname{lag}(u)$ $=\operatorname{lag}(x) \leq(\Lambda-1)(|r|-1) \leq(n-1)(|r|-1)$, giving (i). For $0<\delta<$ $|r|-1$, let $x=z^{-(1 / 2) \delta H}, u=x y$; then $A^{\prime \prime}=u[A]=x\left[A^{\prime}\right]$ is of order $r+\delta<-1$. If $A_{r+\delta}^{\prime \prime}$ is not nilpotent (this is the case when $A_{r}$ is principal nilpotent), then $s=r+\delta, A^{*}=A^{\prime \prime}$. We define $u=x y$; since $\operatorname{tr}(H)=0$, $x$, and hence $u$, belongs to ${ }^{\circ} \mathrm{GL}\left(V\left(\mathscr{F}_{\text {cgt }}\right)\right)$. Moreover $\operatorname{lag}(u)=\operatorname{lag}(x) \leq$ $(\Lambda-1) \delta \leq(n-1)(|r|-|s|)$. If $A_{r+\delta}^{\prime \prime}$ is nilpotent, we go over to $\zeta=z^{1 / b}$ and the $\xi$-lifting $A^{(2)}$ of $A^{\prime \prime} . A^{(2)}$ is of order $\tilde{r}=2 b r+2 a+2 b-1$ while $p l\left(A^{(2)}\right)=2 b p l\left(A^{\prime \prime}\right)+2 b-1=2 b s+2 b-1=\tilde{s}$, say. The induction hypothesis is applicable to $A^{(2)}$ and gives $u^{(2)} \in{ }^{\circ} \mathrm{GL}\left(V\left(\overline{\mathscr{F}}_{5}\right)\right)$ having properties (i) and (ii), with $\tilde{s}$ and $\tilde{r}$ in place of $s$ and $r$ respectively. Let $v \in{ }^{\circ} \mathrm{GL}(V(\overline{\mathscr{F}}))$ correspond to $u^{(2)}$ under an isomorphism of $\overline{\mathscr{F}_{\xi}}$ with $\overline{\mathscr{F}}$ that takes $\zeta$ to $z^{1 / 2 b}$; and let $w=v x y$. It is then easy to check that $w$ has 
property (ii). To verify (i), $\operatorname{lag}(w) \leq \operatorname{lag}(x)+\operatorname{lag}(v) \leq \delta(\Lambda-1)+$ $(1 / 2 b) \operatorname{lag}\left(u^{(2)}\right)$. By induction, $\operatorname{lag}\left(u^{(2)}\right) \leq(n-1)(|\tilde{r}|-|\tilde{s}|)$. We now claim that

$$
|\tilde{r}|-1=2 b(|r|-1-\delta), \quad|\tilde{s}|-1=2 b(|s|-1) .
$$

Assuming this we have, as $\Lambda \leq n$,

$$
\begin{aligned}
\operatorname{lag}(w) & \leq \delta(\Lambda-1)+(n-1)(|r|-|s|-\delta) \\
& \leq \delta(n-1)+(n-1)(|r|-|s|-\delta) \\
& =(n-1)(|r|-|s|) .
\end{aligned}
$$

It remains to check (5.3). But, as $r+\delta<-1$,

$$
\tilde{r}=2 b(r+\delta+1-1 / 2 b)<0,
$$

giving

$$
|\tilde{r}|-1=-2 b(r+\delta+1-1 / 2 b)-1=2 b(|r|-1-\delta) .
$$

Similarly, as $s \leq-1, \tilde{s}=2 b(s+1-1 / 2 b)<0$, giving

$$
|\tilde{s}|-1=-2 b(s+1-1 / 2 b)-1=2 b(|s|-1) .
$$

To prove that this estimate is sharp we give, for arbitrary $n$ and $r$, elements $A, B \in \Omega_{r}$ such that $A \equiv B(n(|r|-1))$ but $p l(A) \neq p l(B)$. We define $A=z^{r} Y_{n}$ and $B=z^{r} Y_{n}+z^{r+m} E_{1 n}$ where $m=n(|r|-1)-1$ and $E_{1 n}$ is as usual the matrix unit. By Corollary 4.6.1, $p l(A)=-1$. But $\delta(B)=m / n=|r|-1-1 / n$ and the same Corollary shows that $p l(B)$ $=-1-1 / n$.

Corollary. If $A$ is regular and $A \equiv B(M)$, then $B$ is also regular.

5.4. The proof of Proposition 5.3 is entirely finitistic and gives an algorithm for determining $p l(A)$ as well as the element $u$ when $r=\operatorname{ord}(A)$ $<-1$. It operates only with the coefficients $A_{r+m}, 0 \leq m<M=$ $n(|r|-1)$, and the main step is the transition from $A$ to $A^{\prime \prime}$ that obviously does not change the principal level. In fact define $A^{\prime \prime}=x y[A]$ where

$$
y=\left(1+z^{M-1} T_{M-1}\right) \cdots\left(1+z T_{1}\right), \quad A^{\prime}=y[A]
$$

and

$$
x=z^{-\delta^{*} H / 2}, \quad \delta^{*}=\min (|r|-1, \delta), \quad \delta=\delta\left(A^{\prime}\right)
$$

as in the proof of Proposition 5.3. If $|r|-1 \leq \delta \leq \infty$ or if $0<\delta<|r|-1$ but $A_{r+\delta}^{\prime \prime}$ is not nilpotent, the algorithm stops; in the first case $A$ is regular, 
in the second it is irregular of principal level $r+\delta<-1 ; A_{r+\delta}^{\prime \prime}$ is given by (4.13). Otherwise we continue with $A^{(2)}$, working only with the coefficients $A_{\tilde{r}+s}^{(2)}, 0 \leq s<\tilde{M}$; from Corollary 4.6 .3 we know that these coefficients are completely and explicitly determined by the $A_{r+s}, 0 \leq s<M$.

This algorithm will end, with the determination of $p l(A)$, in at most $k$ steps where $k$ is the number of nonzero nilpotent orbits. Since $n^{2}-n$ is the maximum dimension for an orbit and all dimensions are even, $k \leq \frac{1}{2}\left(n^{2}-n\right)$.

In particular this algorithm will enable one to decide whether $A \in \Omega_{r}$ is regular or not. We now indicate a method of determining the monodromy of $A$ when $A$ is regular. It is immediate from the above proposition that if $A \in \Omega_{r}$ we can find $u \in{ }^{\circ} \operatorname{GL}\left(V\left(\mathscr{F}_{b}\right)\right)(b \geq 1)$ of the form

$$
u=x^{(m)} y^{(m)} \cdots x^{(1)} y^{(1)},
$$

where the $y^{(i)}$ are of the form (5.4) and the $x^{(i)}$ as in (5.5), such that $B=u[A]$ is of the first kind; $u$ and $B_{-1}$ are entirely determined by the $A_{r+s}, 0 \leq s \leq M$. Let $\tilde{B}$ be the $\zeta$-lifting of $\left(\zeta=z^{1 / b}\right)$. From $\S \S 3.2,3.4$, and 3.6 we know how to find $v \in \operatorname{GL}\left(V\left(\mathscr{F}_{\zeta}\right)\right)$ such that $v[\tilde{B}]=\zeta^{-1} C^{\prime}, C^{\prime} \in$ $\mathfrak{g l}(V)$ and reduced. This requires (\$3.8) only the knowledge of the coefficients $\tilde{B}_{s}, s \leq k\left(\tilde{B}_{-1}\right)=b k\left(B_{-1}\right)$; these in turn depend only on knowing a finite number of additional coefficients of $A$, and are computable in terms of the latter (this involves working with $u^{-1}$ which is easy to compute since the inverses of the $x^{(i)}$ and $y^{(i)}$ are trivial to compute). Write $w=v \hat{u}, C=b^{-1} C^{\prime}$. Then

$$
w[\tilde{A}]=\zeta^{-1}(b C) .
$$

One can then prove that

$$
w\left(e^{2 \pi i / b} \zeta\right) w(\zeta)^{-1}=\boldsymbol{\theta}
$$

is a constant, i.e., an element of $\operatorname{GL}(V)$, that $\theta$ commutes with $C$, and that the conjugacy class

$$
\left[\theta^{-1} \exp 2 \pi i C\right]
$$

is the monodromy of $A$. The proof of this result uses Galois descent arguments and will be given in $\$ 7.4$.

\section{Reduction theory over $\overline{\mathscr{F}}$.}

6.1. In this section we shall use the results of $\S 4.6$ together with the well known spectral "decoupling” lemma (Lemma 6.2) to obtain canonical models in the $\operatorname{GL}(V(\overline{\mathscr{F}}))$-equivalence classes of elements of $\mathfrak{g} \mathfrak{l}(V(\overline{\mathscr{F}}))$. The 
canonical models are of the form

$$
D_{r_{1}} z^{r_{1}}+\cdots+D_{r_{m}} z^{r_{m}}+z^{-1} C
$$

where $r_{1}<r_{2}<\cdots<r_{m}<-1$ are rational numbers, $D_{r_{1}}, \ldots, D_{r_{m}}, C$ are commuting elements in $\mathfrak{g} \mathfrak{I}(V)$, and the $D_{r_{1}}$ are semisimple. The $r_{i}$ are uniquely determined by the corresponding $\mathrm{GL}(V(\overline{\mathscr{F}}))$-equivalence class and are called the canonical levels of the elements of the class. In particular $r_{1}$ is the principal level. Two canonical models, defined by $\left(D_{r_{1}}, \ldots, D_{r_{m}}, C\right)$ and $\left(D_{r_{1}}^{\prime}, \ldots, D_{r_{m}}^{\prime}, C^{\prime}\right)$, are in the same class if and only if for some integer $k \geq 1$

$$
\left(D_{r_{1}}, \ldots, D_{r_{m}}, \exp (2 \pi i k C)\right) \text { and }\left(D_{r_{1}}^{\prime}, \ldots, D_{r_{m}}^{\prime}, \exp \left(2 \pi i k C^{\prime}\right)\right)
$$

are in the same GL( $V$ )-orbit (Theorem 6.5). The connection $D_{r_{1}} z^{r_{1}}$ $+\cdots+D_{r_{m}} z^{r_{m}}$ is called the irregular part of the canonical model $(*)$. It turns out (\$6.6) that the irregular parts of the canonical models in the $\mathrm{GL}(V(\overline{\mathscr{F}}))$-equivalence class $[A]$ of a connection $A \in \mathfrak{g l}(V(\mathscr{F}))$ are completely determined by the coefficients $A_{r+s}, 0 \leq s<n(|r|-1)$, and, in addition, there exists a non-negative integer $k$ effectively computable from the $A_{r+s}, 0 \leq s<n(|r|-1)$ such that the canonical models themselves are determined by $A_{r+s}, 0 \leq s \leq n(|r|-1)+k$; the estimate in the first case is sharp. The proofs of these theorems will yield algorithms for computing canonical models in $[A]$.

The canonical models described here are substantially the ones given by Turrittin [26]. Although his procedure is computational, it is not clear that it leads to sharp estimates as in Theorem 6.6.1. Levelt [16] has treated, from a different point of view, and utilizing cyclic vectors, both the existence and uniqueness of canonical models, but not algorithms or estimates for the number of coefficients. For another treatment of the theory of $\operatorname{GL}(V(\overline{\mathscr{F}}))$-equivalence the reader should refer to Jurkat [11].

6.2. We begin with the classical lemma "decoupling" any $A \in$ $\mathfrak{g} \mathfrak{I}(V(\mathscr{F}))$ of order $r<-1$ along the spectral projections of its leading coefficient.

LEMMA 1. Let

$$
A=z^{r} A_{r}+z^{r+1} A_{r+1}+\cdots \quad(r<-1)
$$

be in $\mathrm{gl}(V(\mathscr{F}))$. Let $\sigma$ be the set of eigenvalues of $A_{r}$; for $\lambda \in \sigma$ let $P_{\lambda}$ be the corresponding spectral projection. Then there is $x \in \mathrm{GL}(V(\theta))_{1}$ such that $A^{\prime}=x[A]$ commutes with all $P_{\lambda}$; in fact, if $X$ is the semisimple component of 
$A_{r}$, there are unique $T_{k} \in$ range of ad $X(k \geq 1)$ such that if

$$
x=\prod_{k=1}^{\infty}\left(1+z^{k} T_{k}\right)=\lim _{m \rightarrow \infty}\left(1+z^{m} T_{m}\right) \cdots\left(1+z T_{1}\right)
$$

then $A^{\prime}=x[A]$ commutes with all $P_{\lambda}$. If $y \in \mathrm{GL}(V(\theta))_{1}$ is such that $A^{\prime \prime}=y[A]$ also commutes with all $P_{\lambda}$, then $y=u x$ and $A^{\prime \prime}=u\left[A^{\prime}\right]$ where $u \in \mathrm{GL}(V(\mathcal{O}))_{1}$ commutes with all $P_{\lambda}$.

Proof. Write $\mathfrak{g}=\mathfrak{g} \mathfrak{l}(V)$ and let $\mathfrak{g}_{X}$ (resp. $\mathfrak{g}^{X}$ ) be the centralizer of ad $X$ (resp. range of ad $X$ ) in $\mathfrak{g}$. Then $S \in \mathfrak{g}_{X}$ if and only if $S$ commutes with all $P_{\lambda}$. Moreover $\mathfrak{g}=\mathfrak{g}_{X} \oplus \mathrm{g}^{X}$, the direct sum is ad $\left(A_{r}\right)$-stable, and $\operatorname{ad}\left(A_{r}\right)$ is invertible on $\mathfrak{g}^{X}$.

The construction of $x$ is essentially a minor variant of $\S 4.3$. Let $A^{(1)}=x^{(1)}[A], x^{(1)}=1+z T_{1}$. Then (cf. (4.3)) $A_{r+1}^{(1)}=A_{r+1}-\operatorname{ad}\left(A_{r}\right)\left(T_{1}\right)$; and the above remark on $\operatorname{ad}\left(A_{r}\right)$ shows that there is a unique $T_{1} \in \mathfrak{g}^{X}$ such that $A_{r+1}^{(1)} \in \mathfrak{g}_{X}$. The rest of the proof is the same as in $\S 4.3$, with $\mathfrak{g}_{X}$ in place of $a$ of that proof. If $y$ is as in the lemma and $u=y x^{-1}$, then $u\left[A^{\prime}\right]=A^{\prime \prime}$ where both $A^{\prime}$ and $A^{\prime \prime}$ commute with $X$ (so that their coefficients are in $\mathfrak{g}_{X}$ ). Write (cf. $§ 1.2$ )

$$
\begin{gathered}
u=\prod_{k=1}^{\infty}\left(1+z^{k} S_{k}\right), \quad u^{(m)}=\prod_{k=m+1}^{\infty}\left(1+z^{k} S_{k}\right), \\
u_{m}=\prod_{k=1}^{m}\left(1+z^{k} S_{k}\right) .
\end{gathered}
$$

Then $A_{r+1}^{\prime \prime}=A_{r+1}^{\prime}-\operatorname{ad}\left(A_{r}\right)\left(S_{1}\right)$, giving $\operatorname{ad}\left(A_{r}\right)\left(S_{1}\right) \in \mathfrak{g}_{X}$; the fact that $\operatorname{ad}\left(A_{r}\right)$ is invertible on $\mathfrak{g}^{X}$ now implies that $S_{1} \in \mathfrak{g}_{X}$. Suppose for some $m \geq 1, S_{1}, \ldots, S_{m} \in g_{X}$. If $B=u_{m}\left[A^{\prime}\right]$, then $B$ commutes with $X$ and $u^{(m)}[B]=A^{\prime \prime}$. As $B_{r}=A_{r}^{\prime \prime}=A_{r}, A_{r+m+1}^{\prime \prime}=B_{r+m+1}-\operatorname{ad}\left(A_{r}\right)\left(S_{m+1}\right)$, giving $S_{m+1} \in \mathfrak{g}_{X}$ as before. Hence all the $S_{j}$ are in $\mathfrak{g}_{X}$, showing that $u$ commutes with $X$.

REMARK. The above proof actually shows that if $u$ has the product representation $\prod_{k=1}^{\infty}\left(1+z^{k} S_{k}\right), A^{\prime \prime}=u\left[A^{\prime}\right]$, and if $A_{r+k}^{\prime \prime}, A_{r+k}^{\prime}$ commute with $X$ for $k<M$, then $S_{k} \in \mathfrak{g}_{X}$ for $1 \leq k<M$ also. Given $A$ we write $x_{A}$ for the element obtained in the lemma whose coefficients in its product representation are all in $\mathrm{g}^{X}$. As in $\$ 4.4$ we now obtain the following corollaries.

Corollary 1. Let $x_{A}=\prod_{k=1}^{\infty}\left(1+z^{k} T_{k}\right)$ be as above. Then $T_{m}(m \geq 1)$ depends only on the $A_{r+s}, 0 \leq s \leq m$; if $A^{\prime}=x_{A}[A], A_{r+m}^{\prime}$ depends only on the $A_{r+s}, 0 \leq s \leq m$. 
Corollary 2. For any integer $M \geq 1$ let $\Omega(A, M)$ be the set of all connections $B$ such that $B_{r+s}=A_{r+s}, 0 \leq s<M$. If

$$
x_{A, M}=y=\left(1+z^{M-1} T_{M-1}\right) \cdots\left(1+z T_{1}\right)
$$

then, for any $B \in \Omega(A, M)$, the connection $B^{\prime}=y[B]$ has the property that $B_{r+s}^{\prime}=A_{r+s}^{\prime}, 0 \leq s<M$. Moreover, $x_{B}$ has the product representation $\Pi_{k}\left(1+z^{k} T_{k}^{\prime}\right)$ where $T_{k}^{\prime}=T_{k}, 1 \leq k<M$.

We shall now prove a variant of this lemma, needed in §6.6. Fix an integer $d \geq 1$ and let $A$ be a connection in $\mathfrak{g l}\left(V\left(\mathscr{F}_{d}\right)\right)$ of order $r<-1$. Write $A$ in the form

$$
A=\sum_{r \leq t \in(1 / d) \mathbf{Z}} A_{t} z^{t}
$$

and assume that for some $M \in(1 / d) \mathbf{Z}$ with $0<M \leq|r|-1$,

$A_{r+s}, 0 \leq s<M$, are semisimple and commute with each other.

LEMMA 2. We can find an element $x \in \mathrm{GL}\left(V\left(\theta_{d}\right)\right)_{1}$ with the property that $A^{\prime}=x[A]$ commutes with all the $A_{r+s}, 0 \leq s<M$, and such that $A_{r+s}^{\prime}=A_{r+s}, 0 \leq s<M$. If $M^{\prime} \geq M$, and if the $A_{r+s}$ for $M \leq s \leq M^{\prime}$ commute with the $A_{r+t}, 0 \leq t<M$, then we can choose $x$ so that $A_{r+s}^{\prime}=A_{r+s}$ for $M \leq s \leq M^{\prime}$ also.

Proof. We may assume $d=1$ by replacing $z$ by $\zeta=z^{1 / d}$. We use induction on $|r|$. If $r=-2$, then $M=1$, and we are in the framework of Lemma 1 and we take $x=x_{A}$. If $A_{r+k}$ for $1 \leq k \leq M^{\prime}$ commutes with $A_{r}$, we have $T_{k} \in \mathrm{g}_{A_{r}}, 1 \leq k \leq M^{\prime}$, by the remark above; as $T_{k} \in \mathrm{g}^{A_{r}}$ also we must have $T_{k}=0$ for such $k$. Suppose now $r<-2$. Let $y=x_{A}, B=y[A]$. Using the remark above once again we find that $B_{r+k}=A_{r+k}, 1 \leq k<M$. Obviously $B$ is the direct sum of connections $B^{(\lambda)} \in \mathfrak{g l}\left(V_{\lambda}(\mathscr{F})\right)$ and

$$
B^{(\lambda)}=\lambda z^{r}+A_{r+1}^{(\lambda)} z^{r+1}+\cdots+A_{r+M-1}^{(\lambda)} z^{r+M-1}+\cdots
$$

where $A_{r+s}^{(\lambda)}$ is the restriction of $A_{r+s}$ to $V_{\lambda}, 1 \leq s<M$. Transforming within $V_{\lambda}$ does not affect the first term. A simple induction argument applied to each $B^{(\lambda)}-\lambda z^{r}$ completes the proof of the first part. The same argument takes care of the second part also.

6.3. We begin with a definition. 
Definition. We shall call any element of $\mathfrak{g} \mathfrak{l}(V(\overline{\mathscr{F}}))$ of the form

$$
D_{r_{1}} z^{r_{1}}+\cdots+D_{r_{m}} z^{r_{m}}+z^{-1} C
$$

canonical if

(i) the $r_{l}$ are distinct rational numbers $<-1$

(ii) $D_{r_{1}}, \ldots, D_{r_{m}}, C \in \mathfrak{g l}(V)$ and commute among themselves

(iii) $D_{r_{1}}, \ldots, D_{r_{m}}$ are semisimple.

If $B \in \mathfrak{g} \mathfrak{l}(V(\overline{\mathscr{F}}))$ is canonical and given by (6.3),

$$
B_{\mathrm{irr}}=D_{r_{1}} z^{r_{1}}+\cdots+D_{r_{m}} z^{r_{m}}
$$

is called the irregular part of $B$, while the $r_{i}$ are called the canonical levels of $B$.

The main result of formal reduction theory is then the following theorem.

TheOrem. (a) Any connection $A \in \mathfrak{g} \mathfrak{l}(V(\overline{\mathscr{F}}))$ is equivalent, under $\mathrm{GL}(V(\overline{\mathscr{F}}))$, to a canonical one; canonical levels of the latter are invariants of the $\operatorname{GL}(V(\overline{\mathscr{F}}))$-equivalence class of $A$.

(b) Two canonical connections

$$
A=D_{r_{1}} z^{r_{1}}+\cdots+D_{r_{m}} z^{r_{m}}+z^{-1} C, \quad A^{\prime}=D_{r_{1}}^{\prime} z^{r_{1}}+\cdots+D_{r_{m}}^{\prime} z^{r_{m}}+z^{-1} C^{\prime}
$$

with the same determinations of the $z^{r_{\jmath}}$ are equivalent under $\operatorname{GL}(V(\overline{\mathscr{F}}))$ if and only if for some integer $k \geq 1$ and some $t \in \mathrm{GL}(V)$,

$$
t \exp (2 \pi i k C) t^{-1}=\exp \left(2 \pi i k C^{\prime}\right), \quad t D_{r_{j}} t^{-1}=D_{r_{j}}^{\prime}, \quad 1 \leq j \leq m .
$$

(c) If $A \in \mathrm{gl}(V(\mathscr{F}))$, its canonical levels are in

$$
\bigcup_{1 \leq q \leq n} q^{-1} \mathbf{Z}
$$

In this section we shall only prove the possibility of reduction to canonical form. The conditions for the equivalence of two canonical forms will be treated in $\S \S 6.4$ and 6.5. The assertion (c) will be established in Proposition 7.6.

Proof of existence of a reduction to canonical form. We use induction on $n$. We may assume that $A$ is irregular. It is also enough to prove the reduction for elements of $\mathfrak{g} \mathfrak{l}(V(\mathscr{F}))$ since we may work with $\zeta=z^{1 / b}$ for suitable integers $b \geq 1$. If $n=1$, we have $A=a_{r} z^{r}+\cdots+a_{-2} z^{-2}+f$, $f \in \mathscr{F}$ being of the first kind. We can find $x \in \mathrm{GL}(1, \mathscr{F})$ such that 
$x[f]=z^{-1} \gamma, \quad \gamma \in \mathbf{C}\left(\exp (2 \pi i \gamma)=\exp \left(2 \pi i a_{-1}\right)\right) . \quad$ As $\quad x[A]=a_{r} z^{r}$ $+\cdots+a_{-2} z^{-2}+\gamma z^{-1}$, we are done.

Assume that $n>1$ and that the theorem is true in dimensions $m<n$. We may also assume that $A \in \mathfrak{g l}(V(\mathscr{F}))$ i.e., all the coefficients of $A$ are of trace 0 . In fact, we can write $A=A^{0}+f 1$ where $A^{0} \in \mathfrak{S} \mathfrak{l}(V(\mathscr{F}))$ and $f=(1 / n) \operatorname{tr}(A)$. By the one-dimensional case treated above we can find $y \in \mathrm{GL}(1, \mathscr{F})$ such that $y[f]$ is in canonical form. So, as $y[A]=A^{0}+$ $y[f] 1$, we may assume that $f$ itself is canonical. Since $x[A]=x\left[A^{0}\right]+f 1$, it is clear that we only need to reduce $A^{0}$.

We call this a trace adjustment. We distinguish two cases.

Case 1. $A_{r}$ has at least two distinct eigenvalues. Let $V=\bigoplus_{\lambda \in \sigma} V_{\lambda}$ be the spectral decomposition of $A_{r}$ with $|\sigma| \geq 2$. By $\$ 6.2$, we can find $x^{\prime} \in \mathrm{GL}(V(\theta))_{1}$ such that $A^{\prime}=x^{\prime}[A]$ is of the form $A^{\prime}=\oplus A_{\lambda}^{\prime}, A_{\lambda}^{\prime} \in$ $\mathfrak{g} \mathfrak{l}\left(V_{\lambda}(\mathscr{F})\right)$. The induction hypothesis is applicable to each $A_{\lambda}^{\prime}$, and we are done.

Case 2. $A_{r}$ has only one eigenvalue. Since $\operatorname{tr}\left(A_{r}\right)=0, A_{r}$ must be nilpotent. We take $A_{r}=Y$ where $\{H, X, Y\}$ is a standard triple and use Proposition 4.3 followed by a trace adjustment to assume that $A_{r+m} \in \mathrm{g}_{X}$ $\cap \mathfrak{g l}(V) m \geq 1$. The argument goes as in Theorem 5.2 based on Proposition 4.6. Let $x$ be as in that proposition and $A^{\prime}=x[A]$. Using downward induction on $\operatorname{dim}\left(\mathrm{GL}(V) \cdot A_{r}\right)$ we may come down to the case when $0<\delta<|r|-1$ and $A_{r+\delta}^{\prime}$ is non-nilpotent. $A_{r+\delta}^{\prime}$ then has at least two distinct eigenvalues, by Corollary 2 to Proposition 4.6. We are thus reduced to Case 1 (after an appropriate $\zeta$-lifting) and the argument is complete.

REMARK. If $r_{\min }=\min _{1 \leq \jmath \leq m} r_{j}, r_{\min }$ is the principal level of $A$. The numbers $r_{i}$ will be called the canonical levels of $A$.

6.4. We shall now examine when two canonical elements are equivalent. We shall actually consider the equivalence between elements a little more general. Let

$$
A=D_{r_{1}} z^{r_{1}}+\cdots+D_{r_{m}} z^{r_{m}}+R=A^{\prime}+R
$$

be an element of $\mathfrak{g} l(V(\overline{\mathscr{F}}))$ such that $A^{\prime}$ is canonical, $R \in \mathfrak{g} \mathfrak{l}(V(\overline{\mathscr{F}}))$ is of the first kind and commutes with all the $D_{r_{j}}$. The $D_{r_{s}}$, being commuting and semisimple, can be simultaneously diagonalized. For $\mathbf{a}=\left(a_{1}, \ldots, a_{m}\right)$ $\in \mathbf{C}^{m}$ let

$$
V(\mathbf{a})=\left\{v \mid v \in V, D_{r_{j}} v=a_{j} v, 1 \leq j \leq m\right\}
$$


Let

$$
\Sigma=\left\{\mathbf{a} \mid \mathbf{a} \in \mathbf{C}^{m}, V(\mathbf{a}) \neq 0\right\}
$$

Then

$$
V=\bigoplus_{\mathbf{a} \in \Sigma} V(\mathbf{a})
$$

Obviously $R$ commutes with the projection $V \rightarrow V($ a) corresponding to the above direct sum. So

$$
R=\bigoplus_{\mathbf{a} \in \Sigma} R(\mathbf{a}) \quad R(\mathbf{a}) \in \mathfrak{g} \mathfrak{l}(V(\mathbf{a})(\overline{\mathscr{F}})) .
$$

All these definitions are relative to $\left(D_{r_{1}}\right)_{1 \leq j \leq m}$; if it is necessary to emphasize this we shall write $V_{A}(\mathbf{a}), \Sigma_{A}$, etc. Note that $\Sigma=(\mathbf{0})$ if and only if $D_{r_{j}}=0$ for all $j$.

LemmA. Let $A$ be as above. Suppose $u \in V(\overline{\mathscr{F}})$ is such that

$$
\dot{u}=A u \text {. }
$$

Then

$$
u \in V(\mathbf{0}) \otimes \overline{\mathscr{F}}
$$

Proof. Let $\mathbf{a} \in \Sigma \backslash(\mathbf{0})$ and let $v \in V(\mathbf{a}) \otimes \overline{\mathscr{F}}$ be the projection of $u$ in $V(\mathbf{a}) \otimes \widetilde{\mathcal{F}}$. Then $v$ satisfies the equation

$$
\dot{v}=A(\mathbf{a}) v, \quad A(\mathbf{a})=a_{1} z^{r_{1}}+\cdots+a_{m} z^{r_{m}}+R(\mathbf{a}) .
$$

Comparison of the orders of $\dot{v}$ and $A(\mathbf{a}) v$ leads to $v=0$.

We now consider

$$
\begin{aligned}
& A=D_{r_{1}} z^{r_{1}}+\cdots+D_{r_{m}} z^{r_{m}}+R_{A}=A^{\prime}+R_{A}, \\
& B=E_{s_{1}} z^{s_{1}}+\cdots+E_{s_{p}} z^{s_{p}}+R_{B}=B^{\prime}+R_{B}
\end{aligned}
$$

where $A^{\prime}$ (resp. $B^{\prime}$ ) is canonical, $R_{A}$ (resp. $R_{B}$ ) is of the first kind and commutes with $A^{\prime}$ (resp. $B^{\prime}$ ).

TheOREM. Let $A$ and $B$ be as above. Then $A$ and $B$ are equivalent under $\mathrm{GL}(V(\overline{\mathscr{F}}))$ if and only if the following conditions are (simultaneously) satisfied:

(i) $m=p, r_{1}=s_{1}, \ldots, r_{m}=s_{m}$;

(ii) with the same choices of the $z^{r_{1}}$ for both $A$ and $B$, there exists $t \in \mathrm{GL}(V)$ such that $t D_{r_{j}} t^{-1}=E_{r_{j}}, 1 \leq j \leq m$; 
(iii) if $\Sigma$ denotes the common spectrum $\Sigma_{A}=\Sigma_{\underline{B}}$ (in virtue of (ii)), then for any $\mathbf{a} \in \Sigma, R_{A}(\mathbf{a})$ and $R_{B}(\mathbf{a})$ are equivalent over $\overline{\mathcal{F}}$.

Moreover, in this case, if $d$ is any integer $\geq 1$ and $y \in \operatorname{GL}\left(V\left(\mathscr{F}_{d}\right)\right)$ is such that $y[A]=B$, then $y$ takes $V_{A}(\mathbf{a}) \otimes \mathscr{F}_{d}$ to $V_{B}(\mathbf{a}) \otimes \mathscr{F}_{d}$ for all $\mathbf{a} \in \Sigma$, and the restriction $y_{\mathbf{a}}$ of $y$ to $V_{A}(\mathbf{a}) \otimes \widetilde{F}_{d}$ takes $R_{A}(\mathbf{a})$ to $R_{B}(\mathbf{a})$ :

$$
y_{\mathbf{a}}\left[R_{A}(\mathbf{a})\right]=R_{B}(\mathbf{a}) \text {. }
$$

Proof. Since the sufficiency of these conditions is obvious we consider only their necessity. Let $y \in \operatorname{GL}(V(\overline{\mathscr{F}}))$ and $y[A]=B$. If $W=\operatorname{End}(V)$, then $y \in W \otimes \overline{\mathscr{F}}$ is a solution of $\dot{u}=C u$ where $C \in \mathfrak{g l}(W)(\overline{\mathscr{F}})$ is such that $C w=B w-w A$. The preceding lemma then shows that $y \in W_{0} \otimes \overrightarrow{\mathcal{F}}$ where $W_{0}$ is the subspace of all $w \in W$ such that $B^{\prime} w-w A^{\prime}=0$. As $y$ is invertible, it follows that $W_{0}$ must contain invertible elements. If $t$ is one such, $t A^{\prime} t^{-1}=B^{\prime}$ and so (i) and (ii) are satisfied. To prove (iii) and the last statement we may, replacing $B$ by $t^{-1} B t$, assume that $A^{\prime}=B^{\prime} . W_{0}$ is then the centralizer of $A^{\prime}$, i.e., of $D_{r_{1}}, \ldots, D_{r_{m}}$, so that $y=\bigoplus_{\mathrm{a}} y_{\mathrm{a}}$ where $y_{\mathrm{a}}$ is in $\operatorname{GL}\left(V_{A}(\mathbf{a})(\overline{\mathscr{F}})\right)$. Clearly we must have $y_{\mathrm{a}}\left[R_{A}(\mathbf{a})\right]=R_{B}(\mathbf{a})$ for all $\mathbf{a}$, so that (iii) and the last assertion follow at once.

6.5. The invariant nature of the canonical levels in the reduction of a connection is clear from Theorem 6.4. Moreover the criterion (b) of Theorem 6.3 is also immediate. We take $R_{A}=z^{-1} C, R_{B}=z^{-1} C^{\prime}$ in Theorem 6.4 and use Proposition 3.10.1 according to which $z^{-1} C_{\mathrm{a}}$ and $z^{-1} C_{\mathrm{a}}^{\prime}$ are equivalent over $\overline{\mathscr{F}}$ if and only if, for some integer $k_{\mathrm{a}} \geq 1$, $\exp \left(2 \pi i k_{\mathrm{a}} C_{\mathbf{a}}\right)$ and $\exp \left(2 \pi i k_{\mathrm{a}}^{\prime} C_{\mathrm{a}}^{\prime}\right)$ are in the same conjugacy class. We omit the details. Theorem 6.3 is thus proved (except for (c)).

Definition. Let $A=D_{r_{1}} z^{r_{1}}+\cdots+D_{r_{m}} z^{r_{m}}+z^{-1} C$ be a canonical element in $\mathfrak{g} \mathfrak{l}(V(\overline{\mathscr{F}}))$. The connection $A^{(s)}=D_{r_{1}} z^{r_{1}}+\cdots+D_{r_{m}}+z^{-1} C_{s}\left(C_{s}\right.$ is the semisimple component of $C$ ) is called the semisimple component of $A$. Two elements of $\mathfrak{g} \mathfrak{l}(V(\overline{\mathscr{F}}))$ are called weakly equivalent if they have canonical forms whose semisimple components are equivalent in $\operatorname{GL}(V(\overline{\mathscr{F}}))$.

For any $B \in \mathfrak{g l}(V(\overline{\mathscr{F}}))$ let $\nabla_{z}^{(B)}=z(d / d z-B)$. If $A=D_{r_{1}} z^{r_{1}}$ $+\cdots+D_{r_{m}} z^{r_{m}}+z^{-1} C$ is canonical, we get the decomposition (analogous to the Jordan decomposition of a linear transformation)

$$
\nabla_{z}^{(A)}=\nabla_{z}^{\left(A^{(s)}\right)}-C_{n}
$$


where, in the above expression, $C_{n}$ stands for the operator of multiplication by $C_{n}$. It is easy to check that $(*)$ is the canonical decomposition of Levelt [16]. Levelt's reduction theory over $\overline{\mathscr{F}}$, in which such canonical decompositions play the main role, is essentially equivalent to the theory developed here. Levelt also obtains similar decompositions for connections in $\mathfrak{g} \mathfrak{l}(V(\mathscr{F}))$.

6.6. In this section we shall examine to what extent the equivalence class (resp. weak equivalence class, irregular part of canonical form) of a connection $A \in \mathfrak{g l}(V(\mathscr{F}))$ can be predicted from the initial segment of its Laurent series. We begin with a generalization of Proposition 5.3.

Proposition 1. Let $A \in \mathfrak{g} \mathfrak{l}(V(\overline{\mathscr{F}}))$ be of order $r<-1$. Let $M=$ $n(|r|-1)$. Then we can find $u \in{ }^{\circ} \mathrm{GL}\left(V\left(\overline{\mathscr{F}}_{\mathrm{cgt}}\right)\right)$ such that

(i) the lag of $u$ is $\leq(n-1)(|r|-1)$;

(ii) there is a canonical element $D_{r_{1}} z^{r_{1}}+\cdots+D_{r_{m}} z^{r_{m}}+z^{-1} C_{A}\left(r_{1}<\right.$ $\left.\cdots<r_{m}<-1\right)$ in $\mathrm{g} \mathfrak{l}(V(\overline{\mathscr{F}}))$ such that

$$
A^{*}=u[A]=D_{r_{1}} z^{r_{1}}+\cdots+D_{r_{m}} z^{r_{m}}+z^{-1} \mathbf{C}_{A}+R_{A}
$$

where $\operatorname{ord}\left(R_{A}\right)>-1$.

Proof. Exactly as in Proposition 5.3, the proof will make it clear that $u$ is convergent. Before beginning we remark that it is enough to prove this proposition for $A \in \mathfrak{g} \mathfrak{l}(V(\mathscr{F}))$; the proposition for $A \in \mathfrak{g} \mathfrak{l}(V(\overline{\mathscr{F}}))$ is then a formal consequence of the technique of $\zeta$-lifting.

Let first $n=1$. Then $A=a_{r} z^{r}+\cdots$ and we need only take $u=1$. Suppose $n>1$. We assume the proposition in dimension $<n$. Assume first that $A_{r}$ has at least two distinct eigenvalues. Let $y=x_{A, M}$ be as in Corollary 6.2.2, let $A^{\prime}=y[A]$ and let $A^{\prime \prime}$ be the connection obtained from $A^{\prime}$ by omitting all powers $z^{p}$ with $p \geq r+M ; A^{\prime}=A^{\prime \prime}+E$ where ord $(E)$ $\geq r+M$ and $A^{\prime \prime}$ commutes with the spectral projections of $A_{r}$. If $\left\{V_{\lambda}\right\}$ are the spectral subspaces of $A_{r}$ with $n_{\lambda}=\operatorname{dim}\left(V_{\lambda}\right)$, and if $A^{\prime \prime(\lambda)}$ is the restriction of $A^{\prime \prime}$ to $V_{\lambda}$, we can find convergent $u^{(\lambda)} \in{ }^{\circ} \operatorname{GL}\left(V_{\lambda}(\overline{\mathscr{F}})\right)$ having properties (i) and (ii), where, in (i), $\left(n_{\lambda}-1\right)(|r|-1)$ appears as the upper bound for $\operatorname{lag}\left(u^{(\lambda)}\right)$; this is possible by the induction hypothesis since $n_{\lambda}<n$. We now use Proposition 1.7.2 to conclude that if $u=\bigoplus_{\lambda} u^{(\lambda)}$, then, $\operatorname{lag}(u) \leq \Sigma_{\lambda} \operatorname{lag}\left(u^{(\lambda)}\right) \leq \Sigma\left(n_{\lambda}-1\right)(|r|-1) \leq(n-2)(|r|-1)$; and $u\left[A^{\prime \prime}\right]$ satisfies (ii). To complete the proof in this case we must only check 
that $u[E]$ has order $\geq 0$. But

$$
\begin{gathered}
\operatorname{ord}(u[E]) \geq r+M-(n-2)(|r|-1) \\
\geq r+2(|r|-1) \geq|r|-2 \geq 0 .
\end{gathered}
$$

We remark that in the remainder of the argument we can use the validity of the proposition, for arbitrary $b \geq 1, r<-1$, whenever $A_{r}$ has at least two distinct eigenvalues.

We may thus assume that $A_{r}=a \cdot 1+Y$ where $Y$ is nilpotent. We now use the induction on the number of canonical levels of $A$. If this number is 0 , we are through by Proposition 5.3. We may thus suppose that $A$ is irregular. If $a \neq 0$ and $\bar{A}=A-a z^{r} 1$, then $r=p l(A)$, and the canonical levels of $\bar{A}$ are exactly those of $A$ with $r$ omitted. So the induction hypothesis is applicable to $\bar{A}$ and proves the proposition for $A$. Suppose now that $a=0$ so that $Y \neq 0$. Let $s=p l(A)$; we have $r<s<$ -1 . Let $u_{1}$ be chosen to satisfy the conditions of Proposition 5.3, and let $A^{*}=u_{1}[A] . A_{s}^{*}$ is not nilpotent, and if it has at least two distinct eigenvalues, the earlier result allows us to find $u^{*} \in{ }^{\circ} \mathrm{GL}(V(\overline{\mathscr{F}}))$ of lag $\leq(n-1)(|s|-1)$ for which $u^{*}\left[A^{*}\right]$ has the specific form (ii). If $u=u^{*} u_{1}$

$$
\operatorname{lag}(u) \leq(n-1)(|s|-1)+(n-1)(|r|-|s|)=(n-1)(|r|-1),
$$

and we are through. If $A_{s}^{*}$ has a single eigenvalue (which must be nonzero), we can write $A^{*}=a^{*} z^{s} \cdot 1+A^{* *}$, with $a^{*} \neq 0$, where $\operatorname{ord}\left(A^{* *}\right)$ $\geq s$ and the number of canonical levels of $A^{* *}$ is one less than the corresponding number for $A^{*}$, i.e., $A$. The induction hypothesis applied to $A^{* *}$ gives $u^{* *}$ having properties (i) and (ii). As before we take $u=u^{* *} u_{1}$ and note that $\operatorname{lag}(u) \leq(n-1)(|r|-1)$. The proof is thus complete.

COROllary. Let $\Omega(A, M)$ be the set of all connections $B \in \mathfrak{g l}(V(\overline{\mathscr{F}}))$ of order $r$ with $B \equiv A\left(\bmod z^{r+M}\right)$. Then, for any $B \in \Omega(A, M)$,

$$
B^{*}=u[B]=D_{r_{1}} z^{r_{1}}+\cdots+D_{r_{m}} z^{r_{m}}+z^{-1} C_{B}+R_{B}
$$

where $\operatorname{ord}\left(R_{B}\right)>-1$. If further $B \equiv A\left(\bmod z^{r+M^{\prime}}\right)$ for some $M^{\prime}>M$, then $C_{B}=C_{A}$.

Proof. Since $\operatorname{lag}(u) \leq(n-1)(|r|-1)$ and $r+M-(n-1)(|r|-1)$ $\geq r+|r|-1=-1, B \equiv A\left(\bmod z^{r+M}\right)$ implies $B^{*} \equiv A^{*}\left(\bmod z^{-1}\right)$, giving the first statement. If $B \equiv A\left(\bmod z^{r+M^{\prime}}\right), B^{*} \equiv A^{*}$ $\left(\bmod z^{-1+M^{\prime}-M}\right)$, proving the second statement. 
The significance of Proposition 1 lies in the fact to be proved below that the connection $D_{r_{1}} z^{r_{1}}+\cdots+D_{r_{m}} z^{r_{m}}+z^{-1}\left(C_{A}\right)_{s}$, the suffix $s$ denoting the semisimple component, is in the weak equivalence class of $A$. To see this let us consider a connection $D$ of the form

$$
D=D_{r_{1}} z^{r_{1}}+\cdots+D_{r_{m}} z^{r_{m}}+z^{-1} C+R=D^{\prime}+R \quad(\operatorname{ord}(R)>-1)
$$

where $D^{\prime}$ is a canonical element of $\mathfrak{g} \mathfrak{l}(V(\overline{\mathscr{F}}))$. Let

$$
D^{\prime(s)}=D_{r_{1}} z^{r_{1}}+\cdots+D_{r_{m}} z^{r_{m}}+z^{-1} C_{s} .
$$

Let $\Sigma$ and $V(\mathbf{a})$ have their usual meaning and let $C_{\mathbf{a}}=C_{V(\mathbf{a})}$. Put

$$
\bar{k}(D)=\bar{k}\left(D^{\prime}\right)=\max _{\mathbf{a} \in \Sigma} \bar{k}\left(C_{\mathbf{a}}\right)
$$

where $\bar{k}\left(C_{\mathbf{a}}\right)$ are the rational numbers $>0$ defined in $\S 3.10$.

Proposition 2. Let notation be as above. Then:

(i) $D$ is in the weak equivalence class of $D^{\prime}$.

(ii) If $E \in \mathfrak{g} \mathfrak{l}(V(\overline{\mathscr{F}}))$ with $E \equiv D\left(\bmod z^{\bar{k}(D)}\right)$, then $E$ is equivalent to $D$ in $\operatorname{GL}(V(\overline{\mathscr{F}}))$.

Proof. Let $d \geq 1$ be an integer such that $D \in \mathfrak{g l}\left(V\left(\mathscr{F}_{d}\right)\right)$. Going over to $\zeta=z^{1 / d}$ we may assume that $d=1$ and $D \in \mathfrak{g} l(V(\mathscr{F}))$, so that all the $r$, are integers $<-1$. In view of Lemma 6.2.2 there is no loss of generality in assuming that $\left[D_{r}, R\right]=0,1 \leq j \leq m$. But then $D$ becomes a direct sum of its restrictions to the spectral spaces $V(\mathbf{a})$, say $D_{\mathbf{a}}$; and, if $\mathbf{a}=\left(a_{1}, \ldots, a_{m}\right) \in \Sigma$,

$$
D_{\mathrm{a}}=a_{1} z^{r_{1}}+\cdots+a_{m} z^{r_{m}}+z^{-1} C_{\mathrm{a}}+R_{\mathrm{a}}, \quad \operatorname{ord}\left(R_{\mathrm{a}}\right) \geq 0 .
$$

The connection $z^{-1} C_{\mathrm{a}}+R_{\mathrm{a}}$ can be reduced to the form $z^{-1} C_{\mathrm{a}}^{\prime}$ in $\mathrm{GL}(V(\mathbf{a})(\mathscr{F}))$; moreover, $\exp \left(2 \pi i\left(C_{\mathbf{a}}^{\prime}\right)_{s}\right)$ and $\exp \left(2 \pi i\left(C_{\mathbf{a}}\right)_{s}\right)$ are conjugate in $\operatorname{GL}(V(\mathbf{a}))$. Since this is true for all $\mathbf{a} \in \Sigma$, we see that $D$ is equivalent over $\operatorname{GL}(V(\mathscr{F}))$ to the connection

$$
D_{r_{1}} z^{r_{1}}+\cdots+D_{r_{m}} z^{r_{m}}+z^{-1} C^{\prime} \quad\left(\left.C^{\prime}\right|_{V(\mathbf{a})}=C_{\mathbf{a}}^{\prime}\right)
$$

and that $\exp \left(2 \pi i C_{s}^{\prime}\right)$ is conjugate to $\exp \left(2 \pi i C_{s}\right)$ via an element of $\mathrm{GL}(V)$ that centralizes $D_{r_{1}}, \ldots, D_{r_{m}}$. In other words, $D^{\prime(s)}$ is in the same weak equivalence class as the connection (6.9) which was equivalent to $D$. This proves (i).

We prove (ii) in three steps. First suppose that both $D$ and $E$ commute with $D_{r_{1}}, \ldots, D_{r_{m}}$. Then $D$ (respectively $E$ ) is the direct sum of its restriction $D_{\mathrm{a}}$ (respectively $E_{\mathrm{a}}$ ) to $V_{\mathrm{a}} . D_{\mathrm{a}}$ and $E_{\mathrm{a}}$ have the same scalar 
irregular part $a_{1} z^{r_{1}}+\cdots+a_{m} z^{r_{m}}$, while Proposition 3.10.2 shows that $D_{\mathbf{a}}^{\prime}=D_{\mathbf{a}}-\left(a_{1} z^{r_{1}}+\cdots+a_{m} z^{r_{m}}\right) \cdot 1$ and $E_{\mathbf{a}}^{\prime}=E_{\mathbf{a}}-\left(a_{1} z^{r_{1}}\right.$ $\left.+\cdots+a_{m} z^{r_{m}}\right) \cdot 1$ are equivalent in $\operatorname{GL}(V(\mathbf{a})(\overline{\mathscr{F}}))$, because $E_{\mathbf{a}}^{\prime} \equiv D_{\mathbf{a}}^{\prime}$ $\left(\bmod z^{\bar{k}\left(C_{\mathrm{a}}\right)}\right) . D$ and $E$ are then obviously equivalent in $\operatorname{GL}(V(\overline{\mathscr{F}}))$.

Next suppose that only $D$ commutes with $D_{r_{1}}, \ldots, D_{r_{m}}$. Choose the integer $\underline{d} \geq 1$ so that $D, E \in \mathfrak{g l}\left(V\left(\mathscr{F}_{d}\right)\right)$ and $\bar{k}(D) \in(1 / d) \mathbf{Z}$. Since $E \equiv D$ $\left(\bmod z^{k(D)}\right)$ it follows that $E_{r+s}=D_{r+s}, r+s<\bar{k}(D)$. By Lemma 6.2.2 there exists $x \in \operatorname{GL}\left(V\left(\theta_{d}\right)\right)_{1}$ such that $E^{\prime}=x[E]$ has the property that $E_{r+s}^{\prime}=E_{r+s}=D_{r+s}, r+s<\bar{k}(D)$, i.e., $E^{\prime} \equiv D\left(\bmod z^{k(D)}\right)$ and $E^{\prime}$ commutes with $D_{r_{1}}, \ldots, D_{r_{m}}$. We are thus reduced to the first case.

Finally we consider the general case: $E \equiv D\left(\bmod z^{k(D)}\right)$. By applying Lemma 6.2 .2 to $D$ with $M=\left|r_{1}\right|-1, M^{\prime}=\left|r_{1}\right|-1$, we see that there exists $x \in \mathrm{GL}\left(V\left(\vartheta_{d}\right)\right)_{1}$ such that $x[D]=D^{\prime}$ commutes with $\left(D_{r_{1}}, \ldots, D_{r_{m}}\right)$ and $D_{r_{1}+s}^{\prime}=D_{r_{1}+s}, r_{1}+s \leq-1$, i.e., $D^{\prime} \equiv D\left(\bmod z^{-1+(1 / d)}\right)$. Note also $\bar{k}(D) \stackrel{k}{=}\left(D^{\prime}\right)$. If $E^{\prime}=x[E]$ it is clear that $E^{\prime} \equiv D^{\prime}\left(\bmod z^{k\left(D^{\prime}\right)}\right)$. So, by the second case above, $E^{\prime}$ is equivalent over $\operatorname{GL}(V(\overline{\mathscr{F}}))$ to $D^{\prime}$ hence $E$ is equivalent to $D$. The proposition is completely proved.

COROllaRY. For $D$ as above, $D_{r_{1}} z^{r_{1}}+\cdots+D_{r_{m}} z^{r_{m}}$ is the irregular part of its canonical form; and $r_{1}, \ldots, r_{m}$ are the canonical levels.

Propositions 1 and 2 lead at once to the following.

THEOREM. Let $r$ be an integer $<-1$ and $A \in \mathfrak{g} l(V(\mathscr{F}))$ a connection of order $r$. Let $M=n(|r|-1)$. If $B \in \mathfrak{g} \mathfrak{l}(V(\mathcal{F}))$ is of order $r$ and $A_{r+s}=B_{r+s}$ for $0 \leq s<M$, then $A$ and $B$ are either both regular or both irregular and have canonical forms with the same irregular part; and this estimate is sharp. If further $A_{r+M}=B_{r+M}$, then $A$ and $B$ are weakly equivalent. Let $u \in$ ${ }^{\circ} \mathrm{GL}\left(V\left(\overline{\mathscr{F}}_{\mathrm{cgt}}\right)\right)$ be as in Proposition 1 , let $A^{*}=u[A]$ and let $\bar{k}=\bar{k}\left(A^{*}\right)$ be defined by (6.8). Then $\bar{k}$ depends only on the $A_{r+s}, 0 \leq s<M$; and if $A_{r+s}=B_{r+s}$ for $0 \leq s \leq M+\bar{k}+1$, then $A$ and $B$ are equivalent over $\operatorname{GL}(V(\overline{\mathscr{F}}))$.

REMARK. Let $A \in \mathfrak{g l}(V(\mathscr{F}))$ be irregular and of order $r<-1$ and let $B$ be a canonical form of $A$. For reasons of (adic) continuity we can find, for any $p>0$, an element $x \in \mathrm{GL}\left(V\left(\overline{\mathscr{F}}_{\text {cgt }}\right)\right)$ such that $x[A]=B+R$ where $R \equiv 0\left(\bmod z^{p}\right)$. Proposition 2 tells us conversely that any connection of the form $B+R, B$ canonical and $R \equiv 0\left(\bmod z^{p}\right)$ necessarily has $B$ as its canonical form if $p$ is sufficiently large. 
6.7. Let $A \in \mathfrak{g l}(V(\mathscr{F}))$ be of order $r<-1$. An examination of the proofs of Proposition 1 above and Proposition 5.3 shows that the elements $u \in{ }^{\circ} \mathrm{GL}(V(\overline{\mathscr{F}}))$ are actually algorithmically constructed, and depend only on the coefficients $A_{r+s}, 0 \leq s<M=n(|r|-1)$. One should note (cf. Proposition 7.6) that as the canonical levels of $A$ are all in $\cup_{1 \leq m \leq n} m^{-1} \mathbf{Z}$, the number of canonical levels of $A$ is at most $(n / 2)(n-1)(|r|-1)$, showing that the inductive process involved in the computation of $u$ as in Proposition 1 will end in a finite number of steps which can be bounded $a$ priori (i.e., depending only on $n$ and $r$ ). The determination of any finite initial segment of $A^{*}$ is thus completely finitistic. Proposition 2 shows that we need to compute $A^{*}$ only $\bmod z^{k}$ where $\bar{k}=\bar{k}\left(A^{*}\right)$ and $d$ is such that $A^{*} \in \mathfrak{g} \mathfrak{l}\left(V\left(\mathscr{F}_{d}\right)\right)$ and $\bar{k} \in(1 / d) \mathbf{Z}$. Let $A^{* *}$ be the $\zeta$-lifting of $A^{*}$ truncated $\bmod z^{k}, \zeta$ being $z^{1 / d}$. Obviously canonical forms of $A^{* *}$ are $\zeta$-liftings of canonical forms of $A$. To determine a canonical form of $A^{* *}$ we follow the method of Lemma 6.2.2 to obtain a connection $A^{\prime} \in \mathfrak{g} \mathfrak{l}\left(V\left(\mathscr{F}_{\xi}\right)\right)$ belonging to the same equivalence class as $A^{* *}$, containing only powers $\zeta^{p}$ with $p<k=\bar{k}\left(A^{* *}\right)$, and commuting with the $D_{r}, 1 \leq j \leq m$; in this procedure we can, thanks to Proposition 6.2.2, always truncate mod $\zeta^{k}$, since at every stage, the connections we deal with begin with the same canonical element of $\mathfrak{g} \mathfrak{l}\left(V\left(\mathscr{F}_{\xi}\right)\right)$. The connection $A^{\prime}$ is then written as a direct sum of connections $A_{\mathrm{a}}^{\prime}$. Each $A_{\mathrm{a}}^{\prime}$ is then reduced to the canonical form by the method of $\S 3$ since the irregular part of $A_{\mathbf{a}}^{\prime}$, being scalar, plays no further role.

\section{Reduction theory over $\mathscr{F}$.}

7.1. In this section we shall discuss the problem of equivalence (of connections) over the field $\mathscr{F}$ itself using the standard technique of Galois descent. The basic problem is to determine, for any integer $b \geq 1$ and any canonical $B \in \mathfrak{g l}\left(V\left(\mathscr{F}_{b}\right)\right)$, the $\operatorname{GL}(V(\mathscr{F}))$-equivalence classes of elements of $\mathfrak{g} l(V(\mathscr{F}))$ that are equivalent to $B$ over $\mathscr{F}_{b}$.

The descent arguments become simpler if we work with the so-called $b$-reduced canonical forms. The significance of $b$-reducedness, which is a generalization of the reducedness of elements of $\mathfrak{g l}(V)$, lies in the fact that the centralizer in $\operatorname{GL}\left(V\left(\mathscr{F}_{b}\right)\right)$ of a canonical $b$-reduced connection in $\mathfrak{g} \mathfrak{l}\left(V\left(\mathscr{F}_{b}\right)\right)$ consists only of constants, i.e., a subgroup of $\operatorname{GL}(V)$. The method of descent is quite explicit and leads to a detailed picture of the $\operatorname{GL}(V(\mathscr{F}))$-equivalence classes in $\mathfrak{g} \mathfrak{l}(V(\mathscr{F}))(\S \S 7.5-7.6)$. For an alternative treatment that is in part based on Turrittin's work [26], see Jurkat [11]. 
7.2. Definition. Let $b$ be any integer $\geq 1$. An element $C \in \mathfrak{g} \mathfrak{l}(V)$ is said to be $b$-reduced if $0 \leq \operatorname{Re}(\lambda)<1 / b$ for all eigenvalues $\lambda$ of $C$. A canonical element

$$
B=D_{r_{1}} z^{r_{1}}+\cdots+D_{r_{m}} z^{r_{m}}+z^{-1} C
$$

of $\mathfrak{g} \mathfrak{l}(V(\overline{\mathscr{F}}))$ is said to be $b$-reduced if $b$ is such that $r_{j} \in(1 / b) \mathbf{Z}$ for all $j$, and if $C$ is $b$-reduced (1-reduced $=$ reduced).

REMARK. If $B \in \mathrm{g} \mathfrak{l}\left(V\left(\mathscr{F}_{b}\right)\right)$ is canonical and $\tilde{B}$ is its $\zeta$-lifting $\left(\zeta=z^{1 / b}\right)$, $B$ is $b$-reduced if and only if $\tilde{B}$ is reduced. Suppose that $B$ above is such that $r_{j} \in(1 / b) \mathbf{Z}$ for all $j$ but that $C$ is not $b$-reduced. Using (3.11) to transform each $z^{-1} C_{\mathbf{a}}(\mathbf{a} \in \Sigma)$ to the form $z^{-1} C_{\mathbf{a}}^{\prime}$ where $C_{\mathbf{a}}^{\prime}$ is $b$-reduced, within $\operatorname{GL}\left(V\left(\mathscr{F}_{b}\right)\right)$, we see that $B$ is $\operatorname{GL}\left(V\left(\mathscr{F}_{b}\right)\right)$-equivalent to a connection $B^{\prime}$ where

$$
B^{\prime}=D_{r_{1}} z^{r_{1}}+\cdots+D_{r_{m}} z^{r_{m}}+z^{-1} C^{\prime}
$$

is canonical and $b$-reduced.

THEOREM. Let $B, B^{\prime}$ be canonical b-reduced, i.e.,

$$
\begin{aligned}
& B=D_{r_{1}} z^{r_{1}}+\cdots+D_{r_{m}} z^{r_{m}}+z^{-1} C, \\
& B^{\prime}=D_{r_{1}}^{\prime} z^{r_{1}}+\cdots+D_{r_{m}}^{\prime} z^{r_{m}}+z^{-1} C^{\prime}
\end{aligned}
$$

where the same choices are made for the $z^{r_{j}}$ in both the expressions. If $y \in \mathrm{GL}\left(V\left(\mathscr{F}_{b}\right)\right)$ and $y[B]=B^{\prime}$, then $y=\tau \in \mathrm{GL}(V)$ and

$$
\tau C \tau^{-1}=C^{\prime}, \quad \tau D_{r_{j}} \tau^{-1}=D_{r_{j}}^{\prime}, \quad 1 \leq j \leq m .
$$

In particular the centralizer of the connection $B$ in $\operatorname{GL}\left(V\left(\mathscr{F}_{b}\right)\right)$ is the subgroup of $\mathrm{GL}(V)$ centralizing $C, D_{r_{1}}, \ldots, D_{r_{m}}$.

Proof. This is immediate from Theorem 6.4; for, $y_{\mathbf{a}}\left[z^{-1} C_{\mathrm{a}}\right]=z^{-1} C_{\mathrm{a}}^{\prime}$, and the $b$-reducedness of $C_{\mathbf{a}}, C_{\mathbf{a}}^{\prime}$ shows that $y_{\mathbf{a}} \in \mathrm{GL}(V(\mathbf{a}))$ (cf. Lemma 3.6).

7.3. The first step is to make more precise the field over which reduction to a canonical form takes place.

Proposition. Let $A \in \mathfrak{g}(V(\mathscr{F}))$ and assume that the canonical levels of $A$ are all in $(1 / b) \mathbf{Z}, b$ being an integer $\geq 1$. Then we can find $x \in \mathrm{GL}\left(V\left(\mathscr{F}_{b}\right)\right)$ such that $x[A]$ is canonical and b-reduced. 
This depends on the following well-known lemma.

Lemma. Let $S$ be any subset of $\mathfrak{g} \mathfrak{l}(V)$ and $\theta \in \mathrm{GL}(V)$ a semisimple element centralizing $S$. Then $\theta$ can be written in the form $\exp (2 \pi i X)$ where $X \in \mathfrak{g} \mathfrak{l}(V)$ is semisimple and centralizes $S$.

Proof. If $H$ is the subgroup of $\mathrm{GL}(V)$ of elements which act as scalars on the eigenspaces of $\theta$, it is clear that $H=\exp \mathfrak{h}$ where $\mathfrak{h}$ is the subalgebra of $\mathfrak{g l}(V)$ whose elements act as scalars on the eigenspaces of $\theta$. Now $\theta \in H$ and the elements of $\mathfrak{h}$ are all semisimple and commute with $S$ since the eigenspaces of $\theta$ are stable under $S$. The lemma is now immediate.

Proof of the Proposition. The proof is a typical application of the Galois descent theory. First of all, it is enough to find $x \in \operatorname{GL}\left(V\left(\mathscr{F}_{b}\right)\right)$ such that $x[A]$ is canonical (cf. Remark in \$7.2). Secondly, going over to $\zeta=z^{1 / b}$ enables us to come down to the case when $b=1$. So we assume that for some $y \in \operatorname{GL}(V(\overline{\mathscr{F}})), y[A]=B$ where

$$
B=D_{r_{1}} z^{r_{1}}+\cdots+D_{r_{m}} z^{r_{m}}+z^{-1} C
$$

is canonical and the $r_{\text {, }}$ are integers. We wish to prove that for a suitable $x \in \mathrm{GL}(V(\mathscr{F})), x[A]$ is canonical. Choose the integer $b \geq 1$ so that $y \in \mathrm{GL}\left(V\left(\mathscr{F}_{b}\right)\right)$. By suitably changing $C$ we may assume that $C$ is $b$-reduced (cf. Remark in §7.2). Identifying $\mu_{b}$ with $\operatorname{Gal}\left(\mathscr{F}_{b} / \mathscr{F}\right)$ and using the fact that $\mu_{b}$ fixes both $A$ and $B$, we see that $y^{\gamma} y^{-1}$ centralizes $B$ for all $\gamma \in \mu_{b}$. Let $\omega=e^{2 \pi i / b}, \zeta=z^{1 / b}$. Then Theorem 7.2 shows that $\hat{y}(\omega \zeta) \hat{y}(\zeta)^{-1}=\theta \in \mathrm{GL}(V)$ and centralizes $C, D_{r_{1}}, \ldots, D_{r_{m}}$. Changing $\zeta$ to $\omega \zeta, \omega^{2} \zeta, \ldots, \omega^{b-1} \zeta$ and multiplying, we get $\theta^{b}=1$. So $\theta$ is semisimple and we can write, in view of the above lemma, $\theta=\exp (2 \pi i X)$ where $X$ is semisimple and centralizes $C, D_{r_{1}}, \ldots, D_{r_{m}}$. Since $\exp (2 \pi i b X)=1$, all ei-

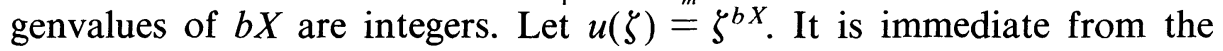
definition of $\zeta^{b X}$ (cf. $\left.\S 1.5\right)$ that $u(\omega \zeta) u(\zeta)^{-1}=\theta$. Hence $u(\zeta)^{-1} \hat{y}(\zeta)=$ $u(\omega \zeta)^{-1} \hat{y}(\omega \zeta)$, showing that $u(\zeta)^{-1} \hat{y}(\zeta)=\hat{x}(\zeta)$ for some $x \in \operatorname{GL}(V(\mathscr{F}))$. But then, $\hat{x}[\tilde{A}]=u^{-1}[\tilde{B}]=\tilde{B}-\zeta^{-1} b X$, so that $x[A]=B-z^{-1} X$. As $B-z^{-1} X$ is canonical, we are through.

7.4. Consider now a canonical element $B \in \mathfrak{g} l(V(\overline{\mathscr{F}}))$. If $r_{1}, \ldots, r_{m}$ are its canonical levels, we have

$$
\begin{aligned}
B & =D_{r_{1}} z^{r_{1}}+\cdots+D_{r_{m}} z^{r_{m}}+z^{-1} C \\
& =B_{\mathrm{irr}}+z^{-1} C \quad\left(r_{J}=s_{j} / b, s_{j} \in \mathbf{Z}\right)
\end{aligned}
$$


for some choices of $z^{r_{1}}, \ldots, z^{r_{m}}$. Let $\Sigma=\Sigma_{B}$ be the spectrum of $\left(D_{r_{1}}, \ldots, D_{r_{m}}\right), V(\mathbf{a})(\mathbf{a} \in \Sigma)$ the spectral subspaces, and for each $\mathbf{a} \in \Sigma$ let

$$
p_{\mathbf{a}}=a_{1} z^{r_{1}}+\cdots+a_{m} z^{r_{m}}
$$

(cf. (6.5) and (6.6)). The set $\Sigma$ as well as the injection $\mathbf{a} \mapsto p_{\mathbf{a}}$ of $\Sigma$ into $\overline{\mathscr{F}}$ depend on the choices of $z^{r_{1}}, \ldots, z^{r_{m}}$; however, the set of all elements $p_{\mathbf{a}}$ is intrinsically defined and coincides with the spectrum of $B_{\text {irr }}$ when it is viewed as a semisimple endomorphism of $V(\overline{\mathscr{F}})$. In fact, if $v \in V(\mathbf{a})$, $B_{\text {irr }} v=p_{\mathbf{a}} v$. Moreover, this spectrum does not change when $B$ is replaced by another canonical element $\operatorname{GL}(V(\overline{\mathscr{F}}))$-equivalent to it. We shall henceforth identify $\Sigma_{B}$ with $\operatorname{spec}\left(B_{1 \mathrm{rr}}\right)$.

Fix an integer $b \geq 1$ and a choice of $\zeta=z^{1 / b}$. for $m / b \in(1 / b) \mathbf{Z}$ let $z^{m / b}=\left(z^{1 / b}\right)^{m}$.

Proposition. Let $\bigodot_{\tilde{g}}(b ; V)$ be the set of all $B \in \mathfrak{g} \mathfrak{l}\left(V\left(\mathscr{F}_{b}\right)\right)$ whose $\operatorname{GL}\left(V\left(\mathscr{F}_{b}\right)\right)$-equivalence class $[B]_{\widetilde{F}_{b}}$ meets $\mathrm{g} \mathfrak{l}(V(\mathscr{F}))$. Let $B$ be as in (7.2) a canonical b-reduced element of $\mathfrak{g} \mathfrak{l}\left(V\left(\mathscr{F}_{b}\right)\right)$. Then the following statements are equivalent:

(i) $B \in \bigodot_{\widetilde{0 F}}(b ; V)$

(ii) $[B]_{\mathscr{F}_{b}}$ is fixed by $\operatorname{Gal}\left(\mathscr{F}_{b} / \mathscr{F}\right)$, i.e., for each $\gamma \in \boldsymbol{\mu}_{b}, \tilde{B}$ and $\gamma \tilde{B}^{\gamma}{ }^{\prime}$ are equivalent with respect to $\mathrm{GL}\left(V\left(\mathscr{F}_{\xi}\right)\right)$

(iii) $\Sigma$ (regarded as a subset of $\left.\mathscr{F}_{b}\right)$ is stable under $\boldsymbol{\mu}_{b}$; and there is a representation $\rho$ of $\boldsymbol{\mu}_{b}$ in $V$, commuting with $C$, such that $\rho$ and $(V(p))_{p \in \Sigma}$ form a system of imprimitivity, i.e.,

$$
\rho(\gamma) C \rho(\gamma)^{-1}=C, \quad \rho(\gamma)(V(p))=V(\gamma \cdot p) \quad\left(\gamma \in \mu_{b}, p \in \Sigma\right)
$$

(i) $\Rightarrow$ (iii). Let $A \in \mathfrak{g l}(V(\mathscr{F}))$ be such that for some $x \in \mathrm{GL}\left(V\left(\mathscr{F}_{b}\right)\right)$, $x[A]=B$. Then $\hat{x}[\tilde{A}]=\tilde{B}$, and from $\S 1.3$ we know that $\hat{x}^{\gamma^{-1}}[\tilde{A}]=\gamma \tilde{B}^{\gamma^{-1}}$ for any $\gamma \in \mu_{b}$. So $\hat{x}^{\gamma^{-1}} \hat{x}^{-1}$ takes $\tilde{B}$ to $\gamma \tilde{B}^{\gamma^{-1}}$. By $\S 7.2$ we must have

$$
\begin{gathered}
\hat{x}(\gamma \zeta) \hat{x}(\zeta)^{-1}=\rho(\gamma) \in \mathrm{GL}(V) \\
\rho(\gamma) C \rho(\gamma)^{-1}=C, \quad \rho(\gamma) D_{r_{1}} \rho(\gamma)^{-1}=\gamma^{s_{J}} D_{r_{r_{1}}}
\end{gathered}
$$

for all $\gamma \in \mu_{b}$. The first of these relations shows that $\rho$ is a representation in $V$. From the second we see that if $p=p_{\mathbf{a}} \in \Sigma$ and $v \in V(p)$, then $D_{r_{j}} \rho(\gamma) v=\gamma^{-s_{j}} \rho(\gamma) D_{r_{j}} v=\gamma^{-s_{\jmath}} a_{\jmath} \rho(\gamma) v$, showing that $\gamma \cdot p \in \Sigma$ and $\rho(\gamma)(V(p))=V(\gamma \cdot p)$. 
(iii) $\Rightarrow$ (ii). This is obvious since (7.4) shows that the $\rho(\gamma)$ satisfy the second set of relations in (7.5) and hence that $\rho(\gamma)[\tilde{B}]=\gamma \tilde{B}^{\gamma^{-1}}\left(\gamma \in \mu_{b}\right)$.

(ii) $\Rightarrow$ (i). By $\$ 7.2$ we can find a $\theta_{\gamma} \in \mathrm{GL}(V)$ for each $\gamma \in \mu_{b}$ such that $\theta_{\gamma}[\tilde{B}]=\gamma \tilde{B}^{\gamma^{-1}}$. We now claim that $\theta_{\gamma}$ can be chosen to be semisimple and to have its $b$ th power equal to 1 . Write $\theta=\theta_{\gamma}$ and $\theta_{s}$ for the semisimple component of $\boldsymbol{\theta}$. As $\operatorname{Ad}\left(\theta_{s}\right)$ is the semisimple component $\operatorname{Ad}(\theta), \theta_{s} C \theta_{s}^{-1}=$ $C, \theta_{s} D_{r_{j}} \theta_{s}^{-1}=\gamma^{s} D_{r_{j}}, 1 \leq j \leq m$. So $\theta_{s}^{b}$ centralizes $\theta_{s}, C, D_{r_{1}}, \ldots, D_{r_{m}}$; Lemma 7.3 allows us to write $\theta_{s}^{b}=\exp (2 \pi i X)$ where $X$ is semisimple and centralizes $\theta_{s}, C, D_{r_{1}}, \ldots, D_{r_{m}}$. Take $\psi=\exp (2 \pi i X / b), \varphi=\psi^{-1} \theta_{s}$. Then $\varphi$ is semisimple, $\varphi^{b}=1$, and $\varphi[\tilde{B}]=\gamma \tilde{B}^{\gamma^{-1}}$. With $\varphi=\theta_{\gamma}$, our claim is proved. Choosing $\theta_{\gamma}$ as above let $\omega \in \mu_{b}$ be primitive and $\theta=\theta_{\omega}$. We assume $V=\mathbf{C}^{n}$ and

$$
\boldsymbol{\theta}=\operatorname{diag}\left(\omega^{k_{1}}, \ldots, \omega^{k_{n}}\right), \quad k_{j} \in \mathbf{Z}
$$

We have $\theta \tilde{B} \theta^{-1}=\omega \tilde{B}^{\omega^{-1}}$. Let $\tilde{B}=\left(\beta_{i j}\right)_{1 \leq i, j \leq n}$. Then

$$
\beta_{i j}(\zeta) \omega^{k_{\imath}-k_{j}-1}=\beta_{l j}(\omega \zeta) \text {. }
$$

Hence, if $\gamma_{i j}(\zeta)=\zeta^{-\left(k_{t}-k_{j}-1\right)} \beta_{i j}(\zeta), \gamma_{l j}(\omega \zeta)=\gamma_{\imath j}(\zeta)$. Hence there is $\alpha_{\imath \jmath} \in$ $\mathscr{F}$ such that $\gamma_{i j}(\zeta)=\alpha_{i j}\left(\zeta^{b}\right)$. Define $D \in \mathfrak{g} \mathfrak{l}(V(\mathscr{F}))$ as the element with the matrix

$$
D=\left(\frac{1}{b z} \alpha_{i j}(z)\right)-\frac{1}{z} \operatorname{diag}\left(\frac{k_{1}}{b}, \ldots, \frac{k_{n}}{b}\right) .
$$

A simple calculation shows $\hat{x}[\tilde{D}]=\tilde{B}$. This proves (i) and hence the proposition.

From now on we fix a canonical $b$-reduced $B \in \mathfrak{g} \mathfrak{l}\left(V\left(\widetilde{F}_{b}\right)\right)$. Let $S(b: B)$ be the set of all systems of imprimitivity

$$
\sigma=\left(\rho,(V(p))_{p \in \Sigma}\right)
$$

where $\rho$ is a representation of $\mu_{b}$ in $V$ commuting with $C$ (cf. (7.4)). It is natural to call two such systems $\sigma_{1}, \sigma_{2}$ equivalent if

$$
\rho_{2}(\gamma)=t \rho_{1}(\gamma) t^{-1} \quad\left(\gamma \in \mu_{b}\right)
$$

for some $t$ in the subgroup $T(B)$ of $\mathrm{GL}(V)$ defined as

$$
\begin{aligned}
& T(B)=\{t \in \mathrm{GL}(V) \mid t(V(p))=V(p) \\
& \text { for all } \left.p \in \Sigma, t C t^{-1}=C\right\} ;
\end{aligned}
$$


of course, in (7.6), $\rho_{i}$ is the representation corresponding to $\sigma_{i}$. It is also obvious that

$$
T(B)=\left\{t \in \mathrm{GL}(V) \mid t C t^{-1}=C, t D_{r_{j}} t^{-1}=D_{r_{j}}, 1 \leq j \leq m\right\} .
$$

Let

$$
\mathcal{S}(b: B)
$$

be the set of all equivalence classes in $S(b: B)$. Finally, let

$$
\begin{array}{r}
\Omega(b: B)=\{A \in \mathfrak{g l}(V(\mathscr{F})) \mid A \text { is equivalent to } B \\
\text { in } \left.\operatorname{GL}\left(V\left(\mathscr{F}_{b}\right)\right)\right\} .
\end{array}
$$

TheOREM. For any $A \in \Omega(b: B)$ let $x$ be any element of $\operatorname{GL}\left(V\left(\mathscr{F}_{b}\right)\right)$ such that $x[A]=B$. Then, for any $\gamma \in \mu_{b}, \rho_{x}(\gamma)=\hat{x}^{\gamma^{-1}} \hat{x}^{-1} \in \mathrm{GL}(V)$; and $\rho_{x}\left(\gamma \rightarrow \rho_{x}(\gamma)\right)$ is a representation of $\boldsymbol{\mu}_{b}$ commuting with $C$ such that $\sigma_{x}=$ $\left(\rho_{x},(V(p))_{p \in \Sigma}\right)$ is a system of imprimitivity. The equivalence class [ $\sigma_{x}$ ] of $\sigma_{x}$ is independent of the choice of $x$ and depends on $A$ only through its $\mathrm{GL}(V(\mathscr{F}))$-equivalence class $[A]$. The map

$$
[A] \mapsto[\sigma]
$$

is then a bijection:

$$
\operatorname{GL}(V(\mathscr{F})) \backslash \Omega(b: B) \approx \delta(b: B) .
$$

Proof. The only non-trivial part of the proof is the surjectivity. This is shown with the help of the following lemma, which is essentially "Hilbert's Theorem 90."

Lemma. Let $\theta \in \mathrm{GL}(V)$ be such that $\theta^{b}=1$. Let $\omega \in \mu_{b}$ be primitive. Then we can find $x \in \mathrm{GL}\left(V\left(\mathscr{F}_{\zeta}\right)\right)$ such that $x^{\omega^{-1}} x^{-1}=\theta$. Moreover we can choose $x$ to be semisimple and commuting with $\theta$.

Proof. Assume $V=\mathbf{C}^{n}$ and $\theta=\operatorname{diag}\left(\lambda_{1}, \lambda_{2}, \ldots, \lambda_{n}\right), \lambda_{J} \in \boldsymbol{\mu}_{b}$. Now $\mathscr{F}_{\zeta}$ is a Galois extension of $\mathscr{F}_{\xi^{b}}$ with $\boldsymbol{\mu}_{b}$ as the Galois group acting as $f(\zeta) \mapsto f\left(\sigma^{-1} \zeta\right)\left(f \in \mathscr{F}_{\zeta}, \sigma \in \boldsymbol{\mu}_{b}\right)$. As $\lambda_{j} \in \mathscr{F}_{\zeta^{b}}$ and $\lambda^{b},=1$, we can write $\lambda_{j}=\left(f_{j}\right)^{\omega^{-1}} f_{j}$ for some $f_{j} \in \mathscr{F}_{\zeta}^{\times}$, by Hilbert's Theorem 90 (see [22], pp. 158-159). Then $x=\operatorname{diag}\left(f_{1}, f_{2}, \ldots, f_{n}\right)$ has all the required properties.

Let $\sigma=\left(\rho,(V(p))_{p \in \Sigma}\right) \in S(b: B)$. By the lemma we can find $x \in$ $\operatorname{GL}\left(V\left(\mathscr{F}_{\zeta}\right)\right)$ such that $x^{\omega^{-1}} x^{-1}=\rho(\omega)=\theta$ where $\omega \in \mu_{b}$ is primitive. Then $x^{\gamma^{-1}} x^{-1}=\rho(\gamma), \gamma \in \mu_{b}$. Let $D=x^{-1}[\tilde{B}]$. We must show that 
$D=\tilde{A}$ for some $A \in \mathfrak{g l}(V(\mathscr{F}))$. Since $\theta=\rho(\omega)$ we must have $\theta[\tilde{B}]=$ $\omega \tilde{B}^{\omega^{-1}}$, so that $x^{\omega^{-1}}[D]=\omega \tilde{B}^{\omega^{-1}}$. But, as $x[D]=\tilde{B}$ we have $x^{\omega^{-1}}\left[\omega D^{\omega^{-1}}\right]$ $=\omega \tilde{B}^{\omega^{-1}}$ also. Hence $D=\omega D^{\omega^{-1}}$, i.e., $D(\omega \zeta)=\omega^{-1} D(\zeta)$. By $\S 1.3$ there exists an $A \in \mathfrak{g l}(V(\mathscr{F}))$ such that $D=\tilde{A}$. This completes the proof.

Let $B \in \mathcal{C}_{\mathscr{G}}(b: V)$. The spectrum $\Sigma$ of $B_{\text {irr }}$ splits into the disjoint orbits

$$
\Sigma_{1}, \Sigma_{2}, \ldots, \Sigma_{q}
$$

under the action of $\operatorname{Gal}(\overline{\mathscr{F}} / \mathscr{F})$, i.e., $\mu_{b}\left(\operatorname{since} \operatorname{Gal}(\overline{\mathscr{F}} / \mathscr{F})\right.$ acts on $\Sigma \subset \mathscr{F}_{b}$ via $\left.\boldsymbol{\mu}_{b}\right)$. Let

$$
V_{J}=V\left(\Sigma_{j}\right)=\bigoplus_{p \in \Sigma_{J}} V(p) .
$$

Each $V_{j}$ is stable under all the $D_{r_{i}}$ and $C$. Let $D_{r_{i}, j}$ and $C_{j}$ be the restrictions to $V_{j}$ of $D_{r_{i}}$ and $C$ respectively. Then we have the following natural decomposition;

$$
B=\bigoplus_{1 \leq j \leq q} B_{j}
$$

where

$$
B_{j}=D_{r_{1}, j} z^{r_{1}}+\cdots+D_{r_{m}, j} z^{r_{m}}+z^{-1} C_{J} .
$$

Of course, for a given $j$, some or all of the $D_{r_{i}, j}$ and $C$, may vanish, so that $B_{j}$ may be regular, even 0 . We call $\left(B_{j}\right)_{1 \leq 1 \leq q}$ the homogeneous constituents of $B$. If $q=1$, i.e., $\Sigma$ is a single orbit, $B$ itself is called homogeneous. The proposition above shows that

$$
B \in \mathcal{C}_{\mathscr{F}}(b: V) \Rightarrow B_{J} \in \mathcal{C}_{\mathscr{F}}\left(b: V_{j}\right) \quad(1 \leq j \leq q) .
$$

Conversely, let $B_{j} \in \mathcal{C}_{\mathscr{F F}}\left(b: V_{j}\right)$ be canonical $b$-reduced homogeneous, with their spectra $\Sigma_{B_{j}}$ disjoint. Then $B=\bigoplus_{j} B$, is in $\bigodot_{0 \bar{f}}(b: V)$ where $V=\oplus_{,} V_{l}$, and the $B_{J}$ are the homogeneous constituents of $B$. Finally we have the following commutative diagram of natural isomorphisms:

$$
\begin{array}{clc}
\mathrm{GL}(V(\mathscr{F})) \backslash \Omega(b: B) & \stackrel{\sim}{\rightarrow} & \delta(b: B) \\
\imath \uparrow & & \imath \uparrow \\
\prod_{1 \leq j \leq q}\left(\mathrm{GL}\left(V_{j}(\mathscr{F})\right) \backslash \Omega\left(b: B_{j}\right)\right) & \stackrel{\sim}{\rightarrow} & \prod_{1 \leq j \leq q} \delta\left(b: B_{j}\right)
\end{array}
$$

The vertical maps are induced respectively by the maps, $\left(A_{1}, \ldots, A_{q}\right) \mapsto A_{1}$ $\oplus \cdots \oplus A_{q}$ of $\prod_{j} \Omega\left(b: B_{j}\right)$ into $\Omega(b: B)$ and $\left(\sigma_{1}, \ldots, \sigma_{q}\right) \mapsto \sigma_{1} \oplus \cdots \oplus \sigma_{q}$ 
of $\prod_{j} S\left(b: B_{j}\right)$ into $S(b: B)$; the map on the right is obviously a bijection so that the vertical map on the left is also bijective.

It is also not difficult to obtain a criterion for deciding when a given canonical $b$-reduced $B \in \mathfrak{g} \mathfrak{l}\left(V\left(\mathscr{F}_{b}\right)\right)$ satisfies the conditions of the preceding Proposition. We leave it to the reader to verify that $B \in \mathcal{C}_{\mathscr{\sigma F}}(b: V)$ if and only if $\Sigma$ is stable under $\boldsymbol{\mu}_{b}$ and for each $\boldsymbol{\mu}_{b}$-orbit $0 \subset \Sigma$, all the $(V(p), C(p))_{p \in 0}\left(C(p)=\left.C\right|_{V(p)}\right)$ are mutually equivalent.

As a first application of the main theorem of this paragraph consider the case when $B$ is regular, i.e., $B=z^{-1} C$ where $C$ is $b$-reduced. If $H$ is the centralizer of $C$ in $\operatorname{GL}(V)$, we have a natural bijection

$$
[A] \mapsto[\theta]_{H}
$$

between the $\operatorname{GL}(V(\mathscr{F}))$-equivalence classes $[A]$ of connections $A \in$ $\operatorname{gl}(V(\mathscr{F}))$ that are $\operatorname{GL}\left(V\left(\mathscr{F}_{b}\right)\right)$-equivalent to $z^{-1} C$, and the conjugacy classes $[\theta]_{H}$ in $H$ of elements $\theta \in H$ for which $\theta^{b}=1$. For a given $A$ we choose any $y \in \operatorname{GL}\left(V\left(\mathscr{F}_{b}\right)\right)$ such that $y[A]=z^{-1} C$; then, with $\omega=e^{2 \pi i / b}$, $y^{\omega^{-1}} y^{-1}=\theta \in \mathrm{GL}(V)$ and defines the associated $[\theta]_{H}$ in $(*)$. To this we add the remark that the GL( $V)$-conjugacy class

$$
\left[\boldsymbol{\theta}^{-1} \exp (2 \pi i C)\right]
$$

is the monodromy of $A$. To see this, we use Lemma 7.3 to write $\theta=\exp (2 \pi i X)$ where $X$ is semisimple and centralizes $C$. As in the proof of Proposition 7.3, bX has only integral eigenvalues, and if $u(\zeta)=\zeta^{b X}$, $u(\zeta)^{-1} \hat{y}(\zeta)=\hat{x}(\zeta)$ for some $x \in \mathrm{GL}(V(\mathscr{F}))$ while $x[A]=z^{-1}(C-X)$. This establishes the above formula for the monodromy of $A$. We recall that this result was used in $§ 5.4$.

As our second application we consider the question of determining the $\operatorname{GL}(V(\mathscr{F})$ )-equivalence classes of connections in $\mathfrak{g} l(V(\mathscr{F}))$ which have a given canonical form. Let

$$
B=D_{r_{1}} z^{r_{1}}+\cdots+D_{r_{m}} z^{r_{m}}+z^{-1} C \in \mathfrak{g l}\left(V\left(\mathscr{F}_{b}\right)\right)
$$

be canonical and let $\Omega(B)$ be the set of all $A \in \mathfrak{g} \mathfrak{l}(V(\mathscr{F}))$ for which $B$ is a canonical form. Let $H$ (resp. $\mathfrak{h}$ ) be the subgroup of GL( $V)$ (resp. subalgebra of $\mathfrak{g l}(V))$ centralized by $D_{r_{1}}, \ldots, D_{r_{m}}$. We select a maximal set of elements $L_{\lambda}(\lambda \in \Lambda)$ of $\mathfrak{h}$ with the following properties:

(a) for each $\lambda \in \Lambda, L_{\lambda}$ is $b$-reduced and there is an integer $k \geq 1$ such that $\exp \left(2 \pi i k L_{\lambda}\right)$ and $\exp (2 \pi i k C)$ are conjugate in $H$;

(b) for $\lambda, \lambda^{\prime} \in \Lambda, \lambda \neq \lambda^{\prime}, L_{\lambda}$ and $L_{\lambda^{\prime}}$ are not conjugate under $H$. For $\lambda \in \Lambda$ let us write

$$
B_{\lambda}=D_{r_{1}} z^{r_{1}}+\cdots+D_{r_{m}} z^{r_{m}}+z^{-1} L_{\lambda} .
$$


It is then clear from Proposition 7.3 that

$$
\Omega(B)=\prod_{\lambda \in \Lambda} \Omega\left(b: B_{\lambda}\right) .
$$

Hence

$$
\operatorname{GL}(V(\mathscr{F})) \backslash \Omega(B)=\prod_{\lambda \in \Lambda}\left(\mathrm{GL}(V(\mathscr{F})) \backslash \Omega\left(b: B_{\lambda}\right)\right) .
$$

Finally, in view of (7.15), it only remains to determine the structure of $\Omega(b: B)$ when $B$ is homogeneous. Fix a

$$
B=D_{r_{1}} z^{r_{1}}+\cdots+D_{r_{m}} z^{r_{m}}+z^{-1} C
$$

which is canonical, $b$-reduced, homogeneous, and belongs to $\bigodot_{\tilde{F}}(b: V)$. Then $\mu_{b}$ acts transitively on $\Sigma$ with isotropy $\mu_{d}$ where $|\Sigma| \cdot d=b$. From the standard theory of systems of imprimitivity one knows that the set $\delta(b: B)$ is naturally bijective with the set of equivalence classes of representations of $\boldsymbol{\mu}_{d}$ in some $V(p)$ that commute with $C(p)=\left.C\right|_{V(p)}$. Fix a generator $\omega_{d}$ of $\mu_{d}$ and let $G(p)$ be the subgroup of $\operatorname{GL}(V(p))$ that centralizes $C(p)$. We then have a natural bijection

$\operatorname{GL}(V(\mathscr{F})) \backslash \Omega(b: B) \approx$ set of conjugacy classes $c$ in $G(p)$ with $c^{d}=$ 1.

In principle these remarks lead to a complete classification of connections in $\mathfrak{g} \mathfrak{l}(V(\mathscr{F}))$ up to $\operatorname{GL}(V(\mathscr{F}))$-equivalence. However there is another way to treat the same question which will allow us to construct explicit models also. We turn to it now.

7.5. For any $A \in \mathfrak{g} \mathfrak{l}(V(\mathscr{F}))$ we define the spectrum of $A$ to be the spectrum of the irregular part of a canonical form of $A$. We shall call $A$ homogeneous if this spectrum is homogeneous in the above sense, i.e., is a single orbit for $\operatorname{Gal}(\overline{\mathscr{F}} / \mathscr{F})$. Let $B$ be a $b$-reduced canonical form in $\mathfrak{g l}\left(V\left(\mathscr{F}_{b}\right)\right)$ such that $A \in \Omega(b: B)$. Then the commutativity of the diagram (7.15) together with the bijective nature of the vertical maps shows that any $A \in \mathfrak{g l}(V(\mathscr{F}))$ is $\operatorname{GL}(V(\mathscr{F}))$-equivalent to $\oplus_{l} A_{i}$ where $V=\oplus_{i} V_{l}$, and $A_{i} \in \mathfrak{g l}\left(V_{i}(\mathscr{F})\right)$ is homogeneous, the $A_{i}$ having disjoint spectra; the $A_{t}$ are determined uniquely upto eigenvalence over $\mathscr{F}$. We shall refer to them as the homogeneous constituents of $A$. Clearly the problem of describing the $\operatorname{GL}(V(\mathscr{F}))$-equivalence classes in $\mathfrak{g} \mathfrak{l}(V(\mathscr{F}))$ reduces to the problem of describing the homogeneous connections.

For any canonical $B \in \mathfrak{g} \mathfrak{l}(V(\overline{\mathscr{F}}))$ given by

$$
B=D_{r_{1}} z^{r_{1}}+\cdots+D_{r_{m}} z^{r_{m}}+z^{-1} C
$$


we define $b(B)$ to be the smallest of the integers $d \geq 1$ such that $r_{j} \in(1 / d) \mathbf{Z}$ for all $j$. We say $B$ is minimal if it is $b(B)$-reduced, i.e., if $C$ is $b(B)$-reduced. If $A \in \mathfrak{g l}(V(\mathscr{F}))$ we define $b(A)$ to be $b(B)$ where $B$ is any canonical form of $A$.

Let $M_{b}$ for any integer $b \geq 1$ be the set of all $B \in \mathfrak{g} \mathfrak{l}\left(V\left(\mathscr{F}_{b}\right)\right)$ with the following properties:

(i) $B$ is canonical, minimal, and $b(B)=b$,

(7.16) (ii) $B$ is equivalent to an element of $\mathfrak{g} l(V(\mathscr{F}))$ under $\operatorname{GL}\left(V\left(\mathscr{F}_{b}\right)\right)$,

(iii) $B$ is homogeneous.

We now fix $B \in M_{b}$ and apply Theorem 7.4 to it. The fact that $b(B)=b$ means $\boldsymbol{\mu}_{b}$ acts with trivial isotropy on $\Sigma$, i.e., $\boldsymbol{\mu}_{b}$ is simply transitive on $\Sigma$. It is well known that in the simply transitive case there is just one isomorphism class of systems of imprimitivity (cf. [23]). Hence the connections in $g \mathfrak{l}(V(\mathscr{F}))$ which are equivalent to $B$ under $\operatorname{GL}\left(V\left(\mathscr{F}_{b}\right)\right)$ form a single $\mathrm{GL}(V(\mathscr{F}))$-equivalence class, which moreover does not change if we replace $B$ by a $B^{\prime} \in M_{b}$ that is $\operatorname{GL}\left(V\left(\widetilde{F}_{b}\right)\right)$-equivalent to it. Finally, if $A \in \mathfrak{g l}(V(\mathscr{F}))$ and is homogeneous, and if $b(A)=b$, Proposition 7.3 guarantees that, for some $B \in M_{b}, A$ will be equivalent to $B$ under $\operatorname{GL}\left(V\left(\mathscr{F}_{b}\right)\right)$. We have thus obtained the following theorem.

Theorem. (a) Any $A \in \mathfrak{g} l(V(\mathscr{F}))$ is equivalent to the direct sum of homogeneous connections in $\mathfrak{g} \mathfrak{l}(V(\mathscr{F}))$ with disjoint spectra, the summands being unique upto permutation and equivalence over $\mathscr{F}$.

(b) For any integer $b \geq 1$, the $\mathrm{GL}(V(\mathscr{F}))$-equivalence classes of homogeneous connections $A$ in $\mathfrak{g} \mathfrak{l}(V(\mathscr{F}))$ with $b(A)=b$ are in natural bijection with the set of $\operatorname{GL}\left(V\left(\mathscr{F}_{b}\right)\right)$-equivalence classes of connections in $M_{b}$; for any $B \in M_{b}$, the $\mathrm{GL}(V(\mathscr{F}))$-equivalence class corresponding to the $\mathrm{GL}\left(V\left(\mathscr{F}_{b}\right)\right)$ equivalence class of $B$ is simply the set of all connections in $\mathfrak{g l}(V(\mathscr{F}))$ that are equivalent to $B$ under $\operatorname{GL}\left(V\left(\mathscr{F}_{b}\right)\right)$.

7.6. To round out the picture we construct explicit models for elements of $M_{b}$ and the corresponding elements of $\mathfrak{g} \mathfrak{l}(V(\mathscr{F}))$.

Fix $b \geq 1$ and take a polynomial $\hat{p}_{0}$ in the variable $\zeta^{-1}$ such that

(i) $\hat{p}_{0}$ is a linear combination of the $\xi^{m}, m \leq-2$;

(ii) $\hat{p}_{0}$ has period exactly $b$ under the substitution $\zeta \mapsto \omega \zeta$ where $\omega=e^{2 \pi l / h}$.

Let $U$ be the $b$-dimensional vector space over $\mathbf{C}$ with basis $\left(e_{l}\right)_{0 \leq l<h}$ and the convention $e_{b}=e_{0}$. Take

$$
V=U \otimes V_{0}
$$


where $V_{0}$ is any finite dimensional vector space over $\mathbf{C}$, and let $C_{0}$ be a $b$-reduced endomorphism of $V_{0}$. We then define $B \in \mathfrak{g} \mathfrak{l}\left(V\left(\mathscr{F}_{b}\right)\right)$ by

$$
B=\operatorname{diag}\left(p_{0}, \omega \cdot p_{0}, \ldots, \omega^{b-1} \cdot p_{0}\right) \otimes 1+z^{-1}\left(1 \otimes C_{0}\right)
$$

where, we write $p_{0}$ for $\hat{p}_{0}\left(z^{1 / b}\right)$, and $\gamma \cdot p_{0}$ is obtained from $p_{0}$ by the substitution $z^{1 / b} \mapsto \gamma^{-1} z^{1 / b}\left(\gamma \in \mu_{b}\right)$.

It is not difficult to verify that $B \in M_{b}$. The spectrum $\Sigma$ is $\left\{p_{0}, \omega \cdot p_{0}, \ldots, \omega^{b-1} \cdot p_{0}\right\}$, the spectral subspaces are given by $V\left(\omega^{r} \cdot p_{0}\right)=$ $\left(\mathbf{C} e_{r}\right) \otimes V_{0}$. If $s \in \mathrm{GL}(U)$ is defined by $s e_{r}=e_{r+1}(0 \leq r \leq b-1)$ and $\rho\left(\omega^{r}\right)=s^{r} \otimes 1,(\rho, V(\cdot))$ is a system of imprimitivity of the type we are discussing. We need to consider no others as we are in the simply transitive context. It is also easy to show that every element of $M_{b}$ is of the above form.

From the explicit form (7.17) we obtain

Proposition. If $A \in \mathfrak{g l}(V(\mathscr{F}))$ is homogeneous, $b(A)$ is a divisor of $n$ and all canonical levels of $A$ are in $b(A)^{-1} \mathbf{Z}$. For an arbitrary $A \in \mathfrak{g} \mathfrak{l}(V(\mathscr{F}))$ the canonical levels of $A$ are in

$$
\bigcup_{1 \leq q \leq n} q^{-1} \mathbf{Z}
$$

and $b(A)$ is a divisor of $\operatorname{lcm}_{1 \leq j \leq n}(j)$.

The element $s$ defined above has eigenvalues $\omega^{j}, 0 \leq j<b$. Let $f_{j}$ be corresponding eigenvectors and let $R$ be the element of $\operatorname{GL}(U)$ defined by $\operatorname{Re}_{j}=f_{j}, 0 \leq j<b$. Using the fact that $\hat{y}(\zeta)=\operatorname{diag}\left(1, \zeta, \ldots, \zeta^{b-1}\right)$ satisfies the relation $\hat{y}(\omega \zeta) \hat{y}(\zeta)^{-1}=\operatorname{diag}\left(1, \omega, \ldots, \omega^{b-1}\right)$, we obtain the following:

$$
\rho(\omega)=\hat{x}(\omega \zeta) \hat{x}(\zeta)^{-1} \quad \text { where } \hat{x}(\zeta)=R \hat{y}(\zeta) \otimes 1 .
$$

The method of $\$ 7.4$ now leads to the following explicit formula for an element $A \in \mathfrak{g} \mathfrak{l}(V(\mathscr{F}))$ which is $\operatorname{GL}\left(V\left(\mathscr{F}_{b}\right)\right)$-equivalent to $B$ :

$$
\text { (7.19) } \begin{aligned}
A= & \left\{y^{-1} R^{-1} \operatorname{diag}\left(p_{0}, \omega \cdot p_{0}, \ldots, \omega^{b-1} \cdot p_{0}\right) R y\right. \\
& \left.-\frac{1}{z} \operatorname{diag}\left(0, \frac{1}{b}, \ldots, \frac{b-1}{b}\right)\right\} \otimes 1 \\
& +\frac{1}{z}\left(1 \otimes C_{0}\right) .
\end{aligned}
$$

Here we are writing $y$ for $\hat{y}$ with $\zeta$ replaced by $z^{1 / b}$. It is not apparent that $A \in \mathfrak{g l}(V(\mathscr{F}))$ but the verification is not difficult. We must also use the same choice of $z^{1 / b}$ in $p_{0}$ as well as $y$. 
For the model $A$ given by (7.19) we consider a fundamental solution $\Phi_{A}$ of the differential equation

$$
d \Phi_{A} / d z=A \Phi_{A} .
$$

If $\Phi_{B}$ is a fundamental solution of

$$
d \Phi_{B} / d z=B \Phi_{B}
$$

we then have, since $A=\left(y^{-1} R^{-1} \otimes 1\right)[B]$,

$$
\begin{aligned}
\Phi_{A} & =\left(y^{-1} R^{-1} \otimes 1\right) \Phi_{B} \\
& =z^{-(1 / b)(\operatorname{diag}(0,1, \ldots, b-1) \otimes 1)} R^{-1} \otimes 1\left(\Phi_{B}\right) .
\end{aligned}
$$

Clearly

$$
\Phi_{B}=e^{\mathrm{d} \operatorname{lag}\left(p_{0}, \omega \cdot p_{0}, \ldots, \omega^{b-1} \cdot p_{0}\right) \otimes 1} \times\left(z^{1 \otimes C_{0}}\right) .
$$

Thus

$$
\Phi_{A}=z^{J} U e^{Q}
$$

with:

$$
\begin{aligned}
& J=-(1 / d) \operatorname{diag}(0,1, \ldots, d-1) \otimes 1+1 \otimes C_{0}, \\
& U=R^{-1} \otimes 1, \\
& Q=\operatorname{diag}\left(p_{0}, \omega \cdot p_{0}, \ldots, \omega^{b-1} \cdot p_{0}\right) \otimes 1
\end{aligned}
$$

which are the same as in [11].

Connections with an Affine Algebraic Structure Group

\section{Regular connections and their monodromy.}

8.1. Let $G$ be an affine algebraic group over $\mathbf{C}$ with Lie algebra $g$. In this section we associate with each regular $A \in \mathfrak{g}(\mathscr{F})$ its monodromy which will be a conjugacy class in $G$ and which will determine the connection upto $G(\mathscr{F})$-equivalence. However one cannot proceed to do this in complete imitation of what was done in $\$ 3$ for $G=G L(V)$ because there are regular connections in $g(\mathscr{F})$ that cannot be transformed to the form $z^{-1} C(C \in \mathfrak{g})$ over $G(\mathscr{F})$ (cf. $\left.§ 8.2\right)$.

In essence our method is to reduce the given regular connection $A$ to the form $z^{-1} C$ over some $\mathscr{F}_{b}$, and then use Galois descent to determine the monodromy of $A$. The monodromy itself is first defined for convergent regular connections in $\mathfrak{g}(\mathscr{F})$ and then extended to all regular connections in $\mathfrak{g}(\mathscr{F})$. It would have been more desirable to give a completely formal treatment. We have not been able to do this. 
8.2. Definition. A connection $A \in \mathfrak{g}(\overline{\mathscr{F}})$ is said to be of the first kind if $\operatorname{ord}(A) \geq-1$, and regular if $x[A]$ is of the first kind for some $x \in G(\overline{\mathscr{F}})$.

Example. Let $G=\operatorname{SL}(2, \mathbf{C}), \mathfrak{g}=\mathfrak{g} \mathfrak{l}(2, \mathbf{C})$. Let $\{H, X, Y\}$ be the standard basis for $g$ (cf. (2.1a)). For any $\alpha \in \mathbf{C}^{\times}$and any integer $m \geq 1$ let

$$
A_{\alpha, m}=z^{-1}\left(\frac{m}{2} H\right)+z^{m-1}(\alpha X) .
$$

We shall now show that $A_{\alpha, m}$ can be transformed to the form $z^{-1} C$, $C \in \mathrm{g}$, over $\operatorname{SL}(2, \mathscr{F})$, if and only if $m$ is even.

It is a trivial calculation to check that

$$
x_{m}\left[A_{\alpha, m}\right]=z^{-1}(\alpha X), \quad x_{m}=\operatorname{diag}\left(z^{-m / 2}, z^{m / 2}\right) .
$$

Suppose now that $m$ is odd, and that for some $y \in \operatorname{SL}(2, \mathscr{F})$ and $C \in$ $\mathfrak{s}(2, \mathbf{C}), y\left[A_{\alpha, m}\right]=z^{-1} C$. Then, with $[\cdot]$ denoting $\operatorname{GL}(2, \mathbf{C})$ conjugacy classes, $[\exp (2 \pi i C)]$ will be the monodromy of $A_{\alpha, m}$ regarded as an element of $\mathfrak{g} l(2, \mathscr{F})$. On the other hand, we have also

$$
u\left[A_{\alpha, m}\right]=z^{-1}\left(\frac{m}{2} 1+\alpha X\right), \quad u=\operatorname{diag}\left(1, z^{m}\right) .
$$

Hence, for some $t \in \mathrm{GL}(2, \mathbf{C}), t \exp (2 \pi i C) t^{-1}=\exp 2 \pi i((m / 2) 1+\alpha X)$ $=-\exp (2 \pi i \alpha X)$. So, writing $C^{\prime}=t C t^{-1}$, and $C^{\prime}=C_{s}^{\prime}+C_{n}^{\prime}$ for its Jordan decomposition, we have $C_{s}^{\prime}, C_{n}^{\prime} \in \mathfrak{s} \backslash(2, \mathbf{C}), C_{n}^{\prime}=\alpha X$ and $\exp \left(2 \pi i C_{s}^{\prime}\right)=$ -1 . As $C_{s}^{\prime}$ is semisimple and centralizes $X, C_{s}^{\prime}=0$, a contradiction.

8.3. Let $A \in \mathrm{g}\left(\widetilde{F}_{\mathrm{cgt}}\right)$ and let $D^{\times}=\{z|0<| z \mid<\rho\}$ be a punctured disc of convergence for $A$. We define $\Pi=\{w \in \mathbf{C} \mid \operatorname{Re}(w)<\log \rho\}$ and regard it as the universal covering of $D^{\times}$for the covering map $w \mapsto z=e^{w}$.

Definition 1. A fundamental solution for $A$ is a holomorphic map $X_{A}=X(\Pi \rightarrow G)$ such that its tangent map $\delta X$ satisfies the equation

$$
(\delta X)(w)=e^{w} A\left(e^{w}\right),
$$

the tangent spaces to $G$ at its various points being canonically identified with g via right translation, so that the tangent map of $X$ may be regarded as a map of $\Pi$ into $g$.

If we regard $G$ as imbedded in $\operatorname{GL}(V)$ and $X$ as a holomorphic map of $\Pi$ into the vector space $\operatorname{End}(V)$, the equation (8.1) becomes the more familiar

$$
\frac{d}{d w} X(w)=e^{w} A\left(e^{w}\right) X(w) .
$$


A fundamental solution for $A$ is automatically a fundamental solution for the classical system (8.2). The converse is also true: if $X$ is a holomorphic map of $\Pi$ into $\operatorname{End}(V)$ satisfying (8.2) such that $X(w) \in G$ for some $w \in \Pi$, then $X$ is a fundamental solution for $A$ in the sense of the above definition. The monodromy of $A$ can now be defined by noting (a) for any fundamental solution $X, w \mapsto X(w+2 \pi i)$ is also a fundamental solution and so for some $\gamma \in G$

$$
X(w+2 \pi i)=X(w) \gamma \quad(w \in \Pi)
$$

and (b) the conjugacy class $[\gamma]_{G}=[\gamma]$ of $\gamma$ in $G$ is independent of the choice of $X$ and depends on $A$ only through its $G\left(\mathscr{F}_{\text {cgt }}\right)$-equivalence class. This class is the monodromy of $A$.

If $H$ is another algebraic group over $\mathbf{C}$ and $\pi(G \rightarrow H)$ a $\mathbf{C}$-morphism, we have the corresponding Lie algebra morphism $\mathrm{g} \rightarrow \mathfrak{h}=\operatorname{Lie}(H)$, denoted by $d \pi$. Then $d \pi(A) \in \mathfrak{h}\left(\mathscr{F}_{\text {cgt }}\right)$ and

$$
\text { Monodromy of } d \pi(A)=\pi(\text { Monodromy of } A) \text {. }
$$

Let $m$ be any integer $\geq 1, D^{\prime \times}=\left\{\zeta|0<| \zeta \mid<\rho^{\prime}=\rho^{1 / m}\right\}$, and let us regard $D^{\prime \times}$ as an $m$-fold covering of $D^{\times}$through the map $\zeta \mapsto z=\zeta^{m}$. The $\zeta$-lifting of $A$ is the connection $\tilde{A} \in \mathfrak{g l}\left(\left(\mathscr{F}_{\zeta}\right)_{\text {cgt }}\right)$ where $\tilde{A}(\zeta)=$ $m \zeta^{m-1} A\left(\zeta^{m}\right)$. Then

$$
\text { Monodromy of } \tilde{A}=(\text { Monodromy of } A)^{m} \text {. }
$$

8.4. If $A \in \mathfrak{g}\left(\mathscr{F}_{\mathrm{cgt}}\right)$ and $x[A]=z^{-1} C$ for some $C \in \mathfrak{g}$ and $x \in G\left(\mathscr{F}_{\text {cgt }}\right)$, it is immediate that $w \mapsto x\left(e^{w}\right)^{-1} \exp (w C)$ is a fundamental solution for $A$, showing that $[\exp (2 \pi i C)]$ is the monodromy of $A$. Let us now call a conjugacy class $c$ in $G$ exponential if $c=[\exp (2 \pi i C)]$ for some $C \in g$. Since not every conjugacy class is exponential, the determination of the monodromy of a regular connection is a little more delicate than in the case of GL( $n)$. The difficulty may be (partially) overcome by going over to $z^{1 / m}=\zeta$ in view of (8.5) and the following result.

Proposition. There is an integer $m \geq 1$ such that if $c$ is a conjugacy class in $G$, then $c^{m}$ is exponential.

Proof. Let $h \in c$ and let $h=h_{s} h_{u}$ be its Jordan decomposition. We write $h_{u}=\exp (2 \pi i N)$ where $N \in \mathrm{g}$ is nilpotent. Let $x=h_{s}$. It is enough to find $L \in \operatorname{center}\left(g_{x}\right)$ and $m \geq 1$ independent of $c$ such that $x^{m}=$ $\exp (2 \pi i L)$. For, if this can be done, we have $[L, N]=0$ as $N \in \mathfrak{g}_{x}$, and hence $h^{m}=x^{m} h_{u}^{m}=\exp (2 \pi i(L+m N))$. We can take $m$ to be the lcm of the numbers $d(\gamma)$ of the lemma below. Note that $x \in G_{x}^{0}$ ([3], p. 271). 
LEMMA. Let $\gamma$ be any semisimple element of $G, Z=\operatorname{center}\left(G_{\gamma}^{0}\right)$. Then the semisimple elements of $Z^{0}$ form a torus $T_{\gamma}$ whose Lie algebra $\mathfrak{I}_{\gamma}$ is contained in center $\left(g_{\gamma}\right)$. If $d(\gamma)=\left[Z: Z^{0}\right]$, then there exists $L \in \mathfrak{I}_{\gamma}$ such that $\gamma^{d(\gamma)}=\exp (2 \pi i L)$. Moreover, as $\gamma$ varies, there are only finitely many $\mathfrak{g}_{\gamma}$ upto conjugacy and hence $d(\gamma)$ varies in a finite set.

Proof. Since $Z^{0}$ is a connected abelian algebraic group, its semisimple elements form a torus ([3], p. 156). As $\gamma \in Z$, the rest is obvious except the finiteness statement. Fix a maximal torus $T$. Since we can move $\gamma$ into $T$ by conjugacy, we may assume that $\gamma$ varies in $T$. We can write $\mathrm{g}=\bigoplus_{\chi} \mathfrak{g}_{\chi}$ where the $\chi$ are distinct characters of $T$ and $T$ acts on $\mathfrak{g}_{\chi}$ as $\chi$. Then $g_{\gamma}$ is the linear span of the $g_{\chi}$ with $\chi(\gamma)=1$.

The main results in the local analytic theory of regular connections depend for their proofs on the result that all conjugacy classes in $\operatorname{GL}(n, \mathbf{C})$ are exponential. Going over to new variables $\zeta=z^{1 / m}$ and using Proposition 1 we may extend all of these results to regular connections in $g\left(\mathscr{F}_{\text {cgt }}\right)$. We formulate these as follows.

(a) Let $H \subset G$ be an algebraic subgroup and let $A \in \mathfrak{h}\left(\mathscr{F}_{\text {cgt }}\right)$ where $\mathfrak{h}=\operatorname{Lie}(H)$. Then for the regularity of $A$ it is immaterial whether $A$ is viewed as an element of $\mathfrak{h}\left(\mathscr{F}_{\text {cgt }}\right)$ or $\mathfrak{g}\left(\mathscr{F}_{\text {cgt }}\right)$. In particular, $A$ is regular if and only if its fundamental solutions satisfy the growth estimate $O\left(|z|^{-N}\right)$ as $z \rightarrow 0$ for some $N \geq 0$ on each sector in the $z$-plane with vertex at the origin and sufficiently small vertex angle.

(b) A regular $A \in \mathfrak{g}\left(\mathscr{F}_{\text {cgt }}\right)$ is determined upto $G\left(\mathscr{F}_{\text {cgt }}\right)$-equivalence by its monodromy.

(c) If $A \in \mathrm{g}\left(\mathscr{F}_{\text {cgt }}\right)$ and is regular, $A$ can be reduced to the form $z^{-1} C$ over $\mathscr{F}_{b}$ if and only if the $b$ th power of its monodromy is exponential. In particular, there is an integer $m \geq 1$ such that all regular connections in $\mathrm{g}\left(\mathscr{F}_{\text {cgt }}\right)$ can be reduced over $G\left(\mathscr{F}_{m, \text { cgt }}\right)$ to the form $z^{-1} C$.

8.5. We begin the treatment of the formal aspects with the analogue of Proposition 3.2. Let $A \in \mathrm{g}(\mathscr{F})$ be of the first kind,

$$
A=z^{-1} A_{-1}+A_{0}+z A_{1}+\cdots \quad\left(A_{l} \in \mathrm{g}\right) .
$$

Let $\sigma=\sigma\left(\operatorname{ad}\left(A_{-1}\right)\right)$ be the spectrum of $\operatorname{ad}\left(A_{-1}\right), \mathfrak{g}_{\lambda}(\lambda \in \sigma)$ the spectral subspaces, and $E_{\lambda}\left(\mathfrak{g} \rightarrow \mathfrak{g}_{\lambda}\right)$ the corresponding projections; $\mathfrak{g}_{\lambda}$ is the eigenspace of $\operatorname{ad}\left(\left(A_{-1}\right)_{s}\right)$ for the eigenvalue $\lambda$.

Definition. $A$ above is said to be aligned if $A_{r} \in \mathfrak{g}_{r+1}$ for any integer $r \geq 0$. Note that in this case (8.6) is a finite sum. 
Proposition. Let $A \in \mathrm{g}(\mathscr{F})$ be of the first kind. Then there is an $x \in G(\theta)_{1}$ such that $x[A]$ is aligned.

Proof. The argument we give is a combination of the ones used in $\S \S 3.2$ and 4.3 , except that we replace expressions of the form $1+z^{m} X$ by $\exp \left(z^{m} X\right)$ to make sure that we work in $G(\mathscr{F})$.

Fix $X \in \mathrm{g}$ and let $\Omega(X)$ be the set of all $A \in \mathrm{g}(\mathscr{F})$ of the first kind with $A_{-1}=X$. Let $\mathrm{g}_{\lambda}$ be as above. Let $\Re(X)$ be the set of all $A \in \Omega(X)$ which are aligned with respect to $X$, i.e., $A=z^{-1} X+A_{0}+\cdots, A_{r} \in \mathfrak{g}_{r+1}$ for all $r \geq 0$; for $B \in \mathscr{B}(X)$ write $\tilde{B}=B(1)-X_{s}$. Let $\mathfrak{n}$ be the subalgebra $\sum_{r \geq 1} \mathfrak{g}_{r}$; it is the Lie algebra of a unipotent (algebraic) subgroup $U$ of $G$. We denote by $\mathcal{T}$ the set of all elements of $G(\mathscr{F})$ of the form

$$
\prod_{r=1}^{\infty} \exp \left(z^{r} T_{r}\right)=\cdots \exp \left(z^{2} T_{2}\right) \exp \left(z T_{1}\right)
$$

where $T_{r} \in \mathrm{g}_{r}$ for all $r \geq 1$. (Note that this product is actually finite.) It can then be shown that: $\mathcal{T}$ is a group, the evaluation $x \mapsto x(1)$ is an isomorphism of $\mathcal{T}$ with $U, \mathcal{T}$ acts on $\Re(X)$, and this action goes over, via the maps $x \mapsto x(1)$ and $B \mapsto \tilde{B}$, to the action of $U$ on $X_{n}+\mathfrak{n}$ by conjugacy ( $X_{n}$ is the nilpotent component of $X$ ). We then have the following theorem. We omit its proof.

Theorem. Let $A \in \Omega(X)$. Then all the aligned models in $\mathscr{B}(X)$ for $A$ form a single T-orbit which depends only on the $G(\theta)_{1}$-equivalence class of $A$. The map, which assigns to the $G(\theta)_{1}$-equivalence class of $A$ the $U$-orbit in $X_{n}+\mathfrak{n}$ that corresponds to the $\mathcal{T}$-orbit in $\mathscr{B}(X)$ of aligned models of $A$, is a bijection:

$$
G(\theta)_{1} \backslash \Omega(X) \stackrel{\sim}{\rightarrow} U \backslash\left(X_{n}+\mathfrak{n}\right) .
$$

8.6. Definition. Let $m \neq 0$ be any integer. Then $X \in \mathrm{g}$ is said to be weakly $m$-reduced if $X_{s}$ and $\exp \left(2 \pi i m X_{s}\right)$ have the same centralizer in $g$. If $m=1$, we say weakly reduced instead of weakly 1-reduced. If $X$ is weakly $m$-reduced for all $m \neq 0$ in $\mathbf{Z}$, we say that $X$ is weakly $\mathbf{Z}$-reduced.

A few comments are perhaps in order concerning this definition. Our aim is to transform an aligned connection to the form $z^{-1} C(C \in \mathrm{g})$ over some extension field $\mathscr{F}_{m}$ and come down to $\mathscr{F}$ via Galois descent. In the case $\mathfrak{g}=\mathfrak{g} \mathfrak{l}(V)$ it was very convenient in descent arguments to work with elements that are $b$-reduced. For arbitrary $g$ it turns out that the notion of 
a weakly $b$-reduced element is a partial substitute and works almost as well (see for instance Lemma 8.7.1).

Proposition. Let $A \in \mathfrak{g}(\overline{\mathscr{F}})$ be regular. Then $A$ is equivalent over $G(\overline{\widetilde{F}})$ to $z^{-1} C$ where $C \in \mathrm{g}$ is weakly $\mathbf{Z}$-reduced.

This depends on the following lemma which will be of use on other occasions also.

LEMma. Let $H \in \mathrm{g}$ be semisimple. Let $\Re(H)$ be the set of all semisimple elements $R \in \mathfrak{g}$ centralizing $\mathfrak{g}_{H}$ and such that $R$ has only rational eigenvalues. If $R \in \mathcal{R}(H)$ is such that $\operatorname{dim}\left(\mathfrak{g}_{H+R}\right) \geq \operatorname{dim}\left(\mathfrak{g}_{H+R^{\prime}}\right)$ for all $R^{\prime} \in \Re(H), H+R$ is weakly $\mathbf{Z}$-reduced.

Proof. It is clear that $\Re(H) \subset \operatorname{center}\left(g_{H}\right)$ so that $\Re(H)$ is an abelian Lie algebra over the rational field. Moreover it is easy to verify that for any $R^{\prime} \in \Re(H), \quad \Re\left(H+R^{\prime}\right) \subset \Re(H)$. Hence $\operatorname{dim}\left(\mathfrak{g}_{H+R}\right) \geq$ $\operatorname{dim}\left(\mathfrak{g}_{H+R+R^{\prime}}\right)$ for all $R^{\prime} \in \mathcal{R}(H+R)$. Replacing $H$ by $H+R$ we may assume that $\operatorname{dim}\left(\mathfrak{g}_{H}\right) \geq \operatorname{dim}\left(\mathfrak{g}_{H+R^{\prime}}\right)$ for all $R^{\prime} \in \mathcal{R}(H)$ and prove that $H$ is weakly $\mathbf{Z}$-reduced. Suppose not. Let $t$ be an integer $\geq 1$ such that $\gamma=\exp (2 \pi i t H)$ has a centralizer in $g$ strictly larger than $\mathfrak{g}_{H}$. By Lemma 8.4 we can find a semisimple $L \in \operatorname{center}\left(\mathfrak{g}_{\gamma}\right)$ and an integer $d \geq 1$ such that $\gamma^{d}=\exp (2 \pi i t d L)$. Then $R=L-H$ lies in $R(H)$; but as $H+R=L$ and $\mathrm{g}_{L} \underset{\neq}{\supset} \mathrm{g}_{H}$, we have a contradiction.

Proof of Proposition. In view of $\$ 8.5$ we may assume that $A$ is convergent and hence (cf. \$8.4) already of the form $z^{-1} D, D \in \mathrm{g}$. Let $D=D_{s}+D_{n}$ be the Jordan decomposition of $D$. By the above lemma we can find $R \in R\left(D_{s}\right)$ such that $D_{s}+R$ is weakly $\mathbf{Z}$-reduced. Since $\left[R, D_{n}\right]$ $=0, C=\left(D_{s}+R\right)+D_{n}$ is the Jordan decomposition of $C=D+R$; and $z^{R}\left[z^{-1} D\right]=z^{-1} C$.

8.7. We shall now use Proposition 8.6 and the descent machinery to complete the theory of monodromy. By that proposition, for any regular $A \in \mathfrak{g}(\mathscr{F})$, we can find an integer $b \geq 1, C \in \mathfrak{g}$ which is weakly $b$-reduced, and $x \in G\left(\mathscr{F}_{b}\right)$, such that $x[A]=z^{-1} C$.

LEMMA 1. Let $D \in g$ be weakly b-reduced and $y \in G\left(\mathscr{F}_{b}\right)$ such that $y\left[z^{-1} D\right]=z^{-1} D$. Then $y \in G_{D}$, the centralizer of $D$ in $G$. 
Proof. Going over to $\zeta=z^{1 / b}$ and replacing $D$ with $b D$, we come

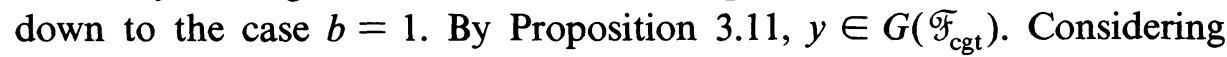
fundamental solutions we have $y\left(e^{w}\right) \exp (w D)=\exp (w D) t_{0}$ for all $w, t_{0}$ $\in G$ being independent of $w$. Changing $w$ to $w+2 \pi i$ we see that $\exp (2 \pi i D)$ centralizes $t_{0}$. If $D=D_{s}+D_{n}$ is the Jordan decomposition of $D$ this means that $D_{n}^{t_{0}}=D_{n}$ and that $\exp \left(2 \pi i D_{s}\right)$ centralizes $t_{0}$. Let $m \geq 1$ be an integer such that $t_{0}^{m} \in\left(G_{\exp \left(2 \pi i D_{s}\right)}\right)^{0}$. Since $D_{s}$ is weakly reduced, $\mathrm{g}_{\exp 2 \pi i D_{s}}=\mathrm{g}_{D_{s}}$ and hence $t_{0}^{m} \in\left(G_{D_{s}}\right)^{0}$. Hence $t_{0}^{m}$ centralizes $D_{s}$, therefore $D$ itself. But then $\left(y\left(e^{w}\right)\right)^{m}=\exp (w D) t_{0}^{m} \exp (-w D)=t_{0}^{m}$, a constant. Therefore $y$ must be a constant, i.e., $y \in G_{D}$.

Fix an integer $b \geq 1$ and a weakly $b$-reduced $C \in g$. Let $R(b: C)$ be the set of all regular elements of $\mathrm{g}(\mathscr{F})$ that are equivalent to $z^{-1} C$ over $G\left(\mathscr{F}_{b}\right)$. It is obvious that $G(\mathscr{F})$ operates on $R(b: C)$. Further, let $\Theta(b: C)$ be the set of all elements $\theta \in G_{C}$ (= centralizer of $C$ in $G$ ) such that $\theta^{b}=1$. Such $\theta$ are always semisimple and $G_{C}$ operates on $\Theta(b: C)$ by conjugation. For $A \in R(b: C)$ (resp. $\theta \in \Theta(b: C)$ ) we write $[A]_{\mathscr{F}}$ (resp. $[\theta]_{C}$ ) for the $G(\mathscr{F})$-equivalence class of $A$ (resp. $G_{C}$-orbit of $\theta$ ). We now proceed exactly as in $\$ 7.4$. Let $A \in R(b: C)$ and let $x \in G\left(\mathscr{F}_{b}\right)$ be such that $x[A]=z^{-1} C$. Write $\omega$ for $e^{2 \pi i / b}$. Then $x\left(\omega z^{1 / b}\right) x\left(z^{1 / b}\right)^{-1}=\theta$ is in $\Theta(b: C)$, and $[\theta]_{C}$ is independent of the choice of $x$ or $z^{1 / b}$. Moreover, $[\theta]_{C}$ depends on $A$ only through $[A]_{\mathscr{F}}$. This allows us to introduce the map

$$
\Phi: G(\mathscr{F}) \backslash R(b: C) \rightarrow G_{C} \backslash \Theta(b: C)
$$

that takes $[A]_{\mathscr{F}}$ to $[\theta]_{C}$ in the above notation. $\Phi$ is one-one. Exactly as in $\$ 7.4$ one can show using Hilbert's Theorem 90 ([22], pp. 158-159) that $\Phi$ is onto. In fact, if $\theta \in \Theta(b: C)$ and $T$ is a torus of $G$ containing $\theta$, we can write $\theta=\exp (2 \pi i L)$ for some $L \in \operatorname{Lie}(T)$. Then $b L$ is semisimple and has only integral eigenvalues so that $\zeta^{b L}=u(\zeta) \in G\left(\mathscr{F}_{\zeta \text {,cgt }}\right)$ and satisfies $u(\omega \zeta) u(\zeta)^{-1}=\theta$. If $x=u\left(z^{1 / b}\right)$ and $A^{\prime}=x^{-1}\left[z^{-1} C\right], \Phi\left(\left[A^{\prime}\right]_{\mathscr{F}}\right)=[\theta]_{C}$.

The last observation shows that any $G(\mathscr{F})$-equivalence class of regular connections in $g(\mathscr{F})$ contains convergent elements, so that its monodromy can be defined as the monodromy of these convergent elements. To complete the picture we must show that the monodromy map is surjective. We shall do this by obtaining an explicit formula for the monodromy of the equivalence class corresponding to $[\theta]_{C}$. Let $A \in \mathrm{g}\left(\mathscr{F}_{\text {cgt }}\right)$ be such that $\Phi\left([A]_{\mathscr{F}}\right)=[\theta]_{C}$. We may choose $x \in G\left(\mathscr{F}_{b, \mathrm{cgt}}\right)$ such that $\hat{x}[\tilde{A}]=\zeta^{-1}(b C)$ and $x(\omega \zeta) x(\zeta)^{-1}=\theta$. If $\tilde{X}$ is a fundamental solution of $\tilde{A}$, $X\left(w \mapsto \tilde{X}\left(b^{-1} w\right)\right)$ is a fundamental solution of $A$, so that 


$$
\begin{array}{rl}
X(w)^{-1} & X(w+2 \pi i)=\tilde{X}\left(b^{-1} w\right)^{-1} \tilde{X}\left(b^{-1}(w+2 \pi i)\right) \\
& =\exp (-w C) \hat{x}\left(e^{b^{-1} w}\right) \hat{x}\left(e^{b^{-1}(w+2 \pi i)}\right)^{-1} \exp ((w+2 \pi i) C) \\
& =\theta^{-1} \exp (2 \pi i C) .
\end{array}
$$

Hence

(8.7) Monodromy of the class corresponding to $[\theta]_{C}=$ $\left[\theta^{-1} \exp (2 \pi i C)\right]$.

The surjectivity of the monodromy map is then a consequence of the following lemma.

LEMMA 2. Let $g \in G$. Then we can write $g$ as $\theta^{-1} \exp (2 \pi i C)$ where, for some integer $b \geq 1, C \in \mathfrak{g}$ is weakly b-reduced while $\theta \in G_{C}$ and $\theta^{b}=1$.

Proof. Let $g=g_{s} \exp (2 \pi i N)$ be the Jordan decomposition of $g$, so that $N \in \mathfrak{g}$ is nilpotent and is in $\mathfrak{g}_{g_{s}}$. By Lemma 8.4 we can find a semisimple $H \in \operatorname{center}\left(\mathfrak{g}_{g_{s}}\right)$ and an integer $d \geq 1$ such that $g_{s}^{d}=$ $\exp (2 \pi$ id $H)$. By Lemma 8.6 we can find $R \in \Re(H)$ such that $H+R$ is weakly Z-reduced. Since $\mathfrak{g}_{g_{s}} \subset \mathfrak{g}_{H}, C=H+R+N$ has $H+R$ (resp. $N$ ) for its semisimple (resp. nilpotent) component. Moreover, as center $\left(\mathfrak{g}_{g_{s}}\right)$ is precisely the centralizer of $\mathfrak{g}_{g_{s}}$ in $\mathfrak{g}, H+R \in \mathfrak{g}_{g_{s}}$. We now define $\theta^{-1}=$ $g_{s} \exp (-2 \pi i(H+R))$ so that $g=\theta^{-1} \exp (2 \pi i C)$. Moreover, if $b$ is a sufficiently large positive integer divisible by $d$ and such that $\exp (-2 \pi i b R)=1$, we have $\theta^{-b}=g_{s}^{b} \exp (-2 \pi i b H)=1$. Finally it is obvious that $\theta \in G_{C}$ and that $C$ is weakly $b$-reduced.

\section{Reduction theory for arbitrary connections.}

9.1. In this section we shall extend the main features of the reduction theory of $\S \S 6$ and 7 to arbitrary connections in $g(\mathscr{F})$. Our methods are essentially the same as before. The definitions of canonical forms and canonical levels remain the same (with $\mathfrak{g}$ in place of $\mathfrak{g} \mathfrak{l}(V)$ ). Exactly as was done in the classical case one can use the techniques of $\S 4$ to obtain the reduction of arbitrary connections to canonical ones when $G$ is reductive. In the general case when $G$ is arbitrary, this method is still applicable to $G / U$ where $U$ is the unipotent radical of $G$, and yields a reduction of the structure groups, from $G$ to a Borel subgroup of $G$. We 
are thus in the solvable case where the reduction to canonical form is much simpler; it is done in $§ 9.2$. We shall also prove that the irregular part of a canonical form of $A$ is determined (upto conjugacy by elements of $G$ ) by the knowledge of the coefficients $A_{r+s}, 0 \leq s<N(|r|-1)$ where $r=\operatorname{ord}(A)$ and $N=1+$ maximum order of the positive roots of a Levi factor of $\mathfrak{g}$. If $\mathfrak{g}=\mathfrak{g l}(V)$, we have $N=\operatorname{dim}(V)$, and this estimate reduces to that of $\S 6$. We conclude in $\S 9.8$ with an application of Galois descent to the problem of determining when two irregular connections in $\mathfrak{g}(\mathscr{F})$ are equivalent to each other over $G(\mathscr{F})$.

9.2. In this paragraph we shall treat the reduction to canonical form under $G(\overline{\mathscr{F}})$ of $A \in \mathrm{g}(\overline{\mathscr{F}})$ when $G$ is solvable. Before proceeding to do this we make a general remark. Let us consider an arbitrary (not necessarily solvable) $G$ and a quotient group $\bar{G}$ of $G$ with $\overline{\mathfrak{g}}=\operatorname{Lie}(\bar{G})$. If $\bar{D} \in \overline{\mathrm{g}}$ is semisimple it is obvious that we can find a semisimple $D \in \mathrm{g}$ with image $\bar{D}$; if in addition $\bar{Z} \in \overline{\mathrm{g}}$ is such that $[\bar{D}, \bar{Z}]=0$, we can choose, in view of the semisimplicity of $\operatorname{ad}(D)$, a $Z \in \mathfrak{g}$ above $\bar{Z}$ with $[D, Z]=0$, with $Z$ semisimple (resp. nilpotent) if $\bar{Z}$ is semisimple (resp. nilpotent). It follows easily that any canonical form in $\overline{\mathfrak{g}}(\overline{\mathscr{F}})$ lifts to a canonical form in $g(\overline{\mathscr{F}})$.

Suppose now that $G$ is solvable and write $U$ for the unipotent radical of $G$. Let $A \in \mathfrak{g}(\overline{\mathscr{F}})$. We shall prove by induction on $\operatorname{dim}(G)$ that $A$ has a reduction to canonical form. If $G$ is abelian so that it is a unipotent group $\times$ torus, the reduction is easy and we omit the details. Suppose $\operatorname{dim}(U)>0 . G$ is the semidirect product of $U$ and a torus $T$. Let $\mathfrak{u}=\operatorname{Lie}(U), \mathfrak{l}=\operatorname{Lie}(T)$. Let $B$ be a connected subgroup of $U$, normal in $G$, of dimension 1 . Since any set of commuting semisimple elements in the Lie algebra of an algebraic group can be imbedded in the Lie algebra of a torus, it follows from the above remark and the induction hypothesis (for $G / V$ ) that $A$ is equivalent to, hence may be assumed to be, a connection of the form $D_{r_{1}} z^{r_{1}}+\cdots+D_{r_{m}} z^{r_{m}}+z^{-1} C+E$ where $D_{r_{1}}, \ldots, D_{r_{m}}, C_{s} \in \mathfrak{l}$, $C_{n} \in \mathfrak{u}, E \in \mathfrak{b}(\overline{\mathscr{F}})$; here $\mathfrak{v}=\operatorname{Lie}(V) . A$ will then be in $H\left(\frac{\vec{F}}{\mathscr{F}}\right)$ for an algebraic group $H \subset G$ with $\operatorname{dim}(H)<\operatorname{dim}(G)$, thus completing the argument, unless (a) $C_{n}$ and $\mathfrak{v}$ span $\mathfrak{u}$ and (b) $T$ is the smallest torus whose Lie algebra contains $C_{s}, D_{r_{1}}, \ldots, D_{r_{m}}$. So $T$ centralizes $C_{n}$ and hence, if $T_{0}$ is the connected centralizer of $V$ in $T, \operatorname{dim}\left(T / T_{0}\right) \leq 1$. Moreover, as $\operatorname{dim}(U)$ $\leq 2, U$ is abelian. A simple argument shows that $G$ is the quotient (by a finite group) of a group $G_{1} \times G_{2}$ where $\operatorname{dim}\left(G_{1}\right) \leq 2$ and $G_{2}$ is abelian. One is thus reduced to the case when $G$ is non-abelian and has dimension 2. $G$ is then seen to be a covering group of the group of matrices of the 
form $\left(\begin{array}{ll}a & b \\ 0 & 1\end{array}\right)$. It is sufficient to prove the theorem for this matrix group and with $\mathscr{F}$ in place of $\overline{\mathscr{F}}$. The element $A \in \mathfrak{g}(\mathscr{F})$ is of the form $\left(\begin{array}{cc}\alpha & \beta \\ 0 & 0\end{array}\right)$ where $\alpha, \beta \in \mathscr{F}$. A simple calculation shows that for $x=\left(\begin{array}{ll}a & b \\ 0 & 1\end{array}\right)$ where $a \in \mathscr{F}^{\times}$, $b \in \mathscr{F}$, we have

$$
x[A]=\left(\begin{array}{cc}
\alpha+\dot{a} a^{-1} & \dot{b}-\left(\alpha+\dot{a} a^{-1}\right) b+a \beta \\
0 & 0
\end{array}\right) .
$$

It is enough to show that for suitable $a, b, \alpha+\dot{a} a^{-1}=\alpha_{\text {irr }}+\theta z^{-1}$ and $\dot{b}-\left(\alpha+\dot{a} a^{-1}\right) b+a \beta=\theta^{\prime} z^{-1}, \boldsymbol{\theta}, \theta^{\prime}$ being constants; here $\alpha_{\text {irr }}$ is the part of $\alpha$ containing the $z^{m}$ with $m \leq-2$, and the condition on $\theta^{\prime}$ is that $\theta^{\prime}=0$ if $\alpha_{\text {irr }} \neq 0$. Using the theory of connections in $\mathfrak{g l}(1, \mathscr{F})$ we can choose $a \in \mathscr{F}^{\times}$such that $\alpha+\dot{a} a^{-1}=\gamma=\alpha_{\text {irr }}+\theta z^{-1}$ where $0 \leq \operatorname{Re}(\theta)<$ 1. It is now a question of choosing $b \in \mathscr{F}$ such that $\dot{b}-\gamma b=-a \beta-\theta^{\prime} z^{-1}$ for some $\theta^{\prime} \in \mathbf{C}$ restricted as above. This is elementary.

9.3. With the single modification that $\exp \left(z^{m} X\right)$ plays the role of $1+z^{m} X$ we can carry over the proofs of $\S \S 4.3,4.4$ and 6.2 to our present context.

We consider a connection $A$ in $g(\mathscr{F})$ given by

$$
A=A_{r} z^{r}+A_{r+1} z^{r+1}+\cdots \quad(r=\operatorname{ord}(A)<-1) .
$$

Let $\mathfrak{a} \subset \mathrm{g}$ be a linear space complementary to the range of $\operatorname{ad}\left(A_{r}\right)$ and let $\mathfrak{b} \subset \mathfrak{g}$ be a linear space complementary to the centralizer $\mathfrak{g}_{A_{r}}$ of $A_{r}$ in $\mathfrak{g}$.

Proposition 1. We can find unique $T_{k} \in \mathfrak{b}(k \geq 1)$ such that if $x_{A}$ is the element of $G(\theta)_{1}$ defined by

$$
x_{A}=\prod_{k=1}^{\infty} \exp \left(z^{k} T_{k}\right)=\lim _{m \rightarrow \infty}\left(\exp \left(z^{m} T_{m}\right) \cdots \exp \left(z T_{1}\right)\right)
$$

and $A^{\prime}=x_{A}[A]$, then $A_{r+m}^{\prime} \in \mathfrak{a}$ for all $m \geq 1$. Moreover, $T_{m}$ depends only on the $A_{r+s}, 0 \leq s \leq m$. In particular, $A_{r+m}^{\prime}$ depends only on the $A_{r+s}$, $0 \leq s \leq m$.

We omit the proof which is an imitation of the arguments of §\$4.3-4.4. These arguments also yield the following corollary.

COROLlary 1. Let $M$ be an integer $\geq 1$ and $\Omega(A, M)$ the set of all connections $B \in \mathrm{g}(\mathscr{F})$ of order $r$ with $A \equiv B(M)$, i.e., $A_{r+s}=B_{r+s}, 0 \leq s$ $<M$. If

$$
x_{A, M}=y=\exp \left(z^{M-1} T_{M-1}\right) \cdots \exp \left(z T_{1}\right)
$$


then all the connections $B^{\prime}=y[B], B \in \Omega(A, M)$ are of order $r$ and have the same coefficients $B_{r+s}^{\prime}=A_{r+s}^{\prime} \in \mathfrak{a}, 0 \leq s<M$. Moreover if

$$
x_{B}=\prod_{k=1}^{\infty} \exp \left(z^{k} S_{k}\right) \quad\left(S_{k} \in \mathfrak{b}\right)
$$

then $S_{k}=T_{k}$ for $1 \leq k<M$.

The spectral splitting Lemma 6.2.1 takes the following form.

Proposition 2. Let $A$ be as above and suppose $X=\left(A_{r}\right)_{s} \neq 0$. Let $\mathfrak{g}_{X}$ be the centralizer of $X$ in $\mathfrak{g}$. Then we can find unique $T_{k}(k \geq 1)$ in range $(\operatorname{ad} X)$ such that if $x_{A} \in G(\theta)_{1}$ is defined by

$$
x_{A}=\prod_{k=1}^{\infty} \exp \left(z^{k} T_{k}\right)
$$

then $A^{\prime}=x_{A}[A]$ belongs to $\mathrm{g}_{X}(\mathscr{F})$. Moreover, $T_{m}$ and $A_{r+m}^{\prime}$ depend only on the $A_{r+s}, 0 \leq s \leq m$.

REMARK. If $X \notin$ center $(\mathfrak{g}), \operatorname{dim}\left(\mathfrak{g}_{X}\right)<\operatorname{dim}(\mathfrak{g})$. This proposition then allows us to use induction on $\operatorname{dim}(g)$.

Corollary 2. Let $\Omega(A, M)$ be as in Corollary 1 . If

$$
x_{A, M}=y=\exp \left(z^{M-1} T_{M-1}\right) \cdots \exp \left(z T_{1}\right)
$$

then, for any $B \in \Omega(A, M), B^{\prime}=y[B]$ has the property $B_{r+s}^{\prime}=A_{r+s}^{\prime}$, $0 \leq s<M$. Moreover, if $x_{B}=\prod_{k=1}^{\infty} \exp \left(z^{k} S_{k}\right)$, then $S_{k}=T_{k}, 1 \leq k<M$.

9.4. The crucial step in reduction theory is when $G$ is reductive. The formulations and proofs of $\$ 4.6$ go over without any change. For ease of reference we put down

Proposition. Let $Y \neq 0$ be a nilpotent in $[\mathrm{g}, \mathfrak{g}], \mathfrak{g}$ being reductive. Let $A=A_{r} z^{r}+\cdots \in \mathfrak{g}(\mathcal{F})$ with $r<-1$ and $A_{r}=Y$. Assume that the conditions (4.9) are satisfied. Then (a)-(c) of Proposition 4.6 and Corollary 3 are true (with $\mathrm{gl}(V), \mathrm{GL}(V), \ldots$ replaced by $\mathfrak{g}, G, \ldots)$.

9.5. Let now $G$ be an arbitrary affine algebraic group.

Theorem. (a) Any $A \in g(\overline{\mathscr{F}})$ is equivalent under $G(\overline{\mathscr{F}})$ to a canonical form. The canonical levels of the latter are invariants of the $G(\overline{\mathscr{F}})$-equivalent class of $A$. 
(b) Let

$$
\begin{aligned}
& A=D_{r_{1}} z^{r_{1}}+\cdots+D_{r_{m}} z^{r_{m}}+z^{-1} C, \\
& A^{\prime}=D_{r_{1}}^{\prime} z^{r_{1}}+\cdots+D_{r_{m}}^{\prime} z^{r_{m}}+z^{-1} C^{\prime}
\end{aligned}
$$

be canonical forms with the same determinations of the $z^{r_{j}}$ 's. Then $A$ and $A^{\prime}$ are equivalent over $G(\overline{\mathscr{F}})$ if and only if for some integer $k \geq 1$ and $t \in G$,

$$
\begin{aligned}
& t \exp (2 \pi i k C) t^{-1}=\exp \left(2 \pi i k C^{\prime}\right), \\
& \operatorname{Ad}(t)\left(D_{r_{j}}\right)=D_{r_{j}}^{\prime}, \quad 1 \leq j \leq m .
\end{aligned}
$$

Proof. In this paragraph we prove (a). We use induction on $\operatorname{dim}(G)$. Fix $A \in g(\overline{\mathscr{F}})$. If the unipotent radical $U$ of $G$ has positive dimension, the induction hypothesis applied to the image of $A$ in $\overline{\mathfrak{g}}(\overline{\mathscr{F}})(\overline{\mathrm{g}}=\operatorname{Lie}(G / U))$ shows that $A$ is equivalent to, hence may be assumed to be, a connection of the form $B+E$, where $B$ is canonical and $E \in \mathfrak{u}(\overline{\mathscr{F}})$, $\mathfrak{u}$ being $\operatorname{Lie}(U)$. Hence $B+E \in \mathfrak{b}(\overline{\mathscr{F}})$ where $\mathfrak{b}$ is a Borel subalgebra of $\mathfrak{g}$. We are through by $\$ 9.2$. So we may assume $G$ to be reductive. Using the splitting of a reductive group as a torus $X$ semisimple group (locally) we come down to the case when $G$ is semisimple. If $r=\operatorname{ord}(A)<-1$ and $X=\left(A_{r}\right)_{s} \neq 0$, Proposition 9.3 and the induction hypothesis complete the argument as $\operatorname{dim}\left(\mathfrak{g}_{X}\right)<\operatorname{dim}(\mathfrak{g})$. If $A_{r}$ is nilpotent, Proposition 9.4 and a downward induction on $\operatorname{dim}\left(G \cdot A_{r}\right)$ prove the required result.

9.6. It remains to examine the conditions for equivalence of canonical forms. The sufficiency of the conditions is trivial. For proving the necessity, we may, by enlarging $V$ if necessary, assume that $G$ is imbedded in $\operatorname{SL}(V)$. In particular, $G$ is closed in $\operatorname{End}(V)$. Let $E=\operatorname{End}(V)$.

We shall show first that if $x \in G(\overline{\mathscr{F}})$ and $x[A]=A^{\prime}$, then

$$
\operatorname{Ad}(x)\left(D_{r_{j}}\right)=D_{r_{j}}^{\prime}, \quad 1 \leq j \leq m, \quad x\left[z^{-1} C\right]=z^{-1} C^{\prime} .
$$

In fact, for any such $x$, it is immediate from Theorem 6.4 that

$$
D_{r_{j}}^{\prime} x=x D_{r_{j}} \quad(1 \leq j \leq m) .
$$

Let us now consider the subspace $J$ (resp. $J(\overline{\mathscr{F}}))$ of $E$ (resp. $E(\overline{\mathscr{F}}))$ of all $y$ satisfying $D_{r_{j}}^{\prime} y-y D_{r_{j}}=0,1 \leq j \leq m ; J(\overline{\mathscr{F}})$ is spanned by $J$ over $\overline{\mathscr{F}}$. The above relations may now be interpreted as the statement that the closed algebraic varieties $J$ and $G$ have a common point over $\overline{\mathscr{F}}$. As these varieties are already defined over $\mathbf{C}$ they must have a common point over $\mathbf{C}$. Hence, for some $s \in G, D_{r_{j}}^{\prime}=s D_{r_{j}} s^{-1}, 1 \leq j \leq m$. Replacing $A^{\prime}$ with 
$s^{-1}\left[A^{\prime}\right]=s^{-1} A^{\prime} s$ we may assume that $D_{r_{j}}^{\prime}=D_{r_{j}}, 1 \leq j \leq m$. Then $x D_{r_{j}} x^{-1}$ $=D_{r}, 1 \leq j \leq m$, and so the relation $x[A]=A^{\prime}$ reduces to $x\left[z^{-1} C\right]=$ $z^{-1} C^{\prime}$. Let $Z$ be the centralizer of the $D_{r_{j}}(1 \leq j \leq m)$. Then $Z$ is a connected algebraic subgroup of $G$, and $x \in Z(\overline{\mathscr{F}})([3]$, p. 271). Obviously $C$ and $C^{\prime}$ belong to $z=\operatorname{Lie}(Z)$; and $x\left[z^{-1} C\right]=z^{-1} C^{\prime}$ means that $z^{-1} C$ and $z^{-1} C^{\prime}$ are equivalent in some $Z\left(\mathscr{F}_{k}\right)$. So $\exp (2 \pi i k C)$ and $\exp \left(2 \pi i k C^{\prime}\right)$ must be in the same conjugacy class in $Z$. This proves (9.1) and finishes the proof of Theorem 9.5.

9.7. Let $U$ be the unipotent radical of $G, G / U=\bar{G}$, and $\overline{\mathrm{g}}=\operatorname{Lie}(\bar{G})$. We recall the definition of ord $(\overline{\mathrm{g}})(\mathrm{cf} .(2.20))$ and define

$$
N(\mathfrak{g})=1+\operatorname{ord}(\overline{\mathfrak{g}}) \text {. }
$$

Our aim in this paragraph is to prove the following theorem.

THEOREM. Let $r$ be an integer $<-1$, and let

$$
M=M(\mathfrak{g}, r)=N(\mathfrak{g})(|r|-1) .
$$

If $A$ and $B$ are two connections in $\mathfrak{g}(\mathscr{F})$ of order $r$ such that $A_{r+m}=B_{r+m}$ for $0 \leq m<M$, then either $A$ and $B$ are both regular, or both are irregular and the irregular parts of their canonical forms are conjugate under $G$.

Proof. We prove the theorem by induction on $\operatorname{dim}(G)$. Suppose first that $U \neq(1)$ so that $\operatorname{dim}(\bar{G})<\operatorname{dim}(G)$. Let $A, B$ be as in the theorem. We choose a Levi subgroup $H$ of $G$ and write $\mathfrak{h}=\operatorname{Lie}(H)$. It is easily seen that $A$ (resp. $B$ ) is conjugate over $G(\overline{\mathscr{F}})$ to a canonical connection $A^{\prime}=D_{r_{1}} z^{r_{1}}+\cdots+D_{r_{m}} z^{r_{m}}+z^{-1} C_{A}$ (resp. $B^{\prime}=E_{s_{1}} z^{s_{1}}+\cdots+E_{s_{p}} z^{s_{p}}+$ $z^{-1} C_{B}$ ) where $r_{1}<\cdots<r_{m}<-1$ (resp. $s_{1}<\cdots<s_{p}<-1$ ) and $D_{r_{i}}, E_{s_{j}}$ belong to $\mathfrak{h}$. If $\bar{A}$ (resp. $\bar{B}$ ) is the element of $\overline{\mathfrak{g}}(\mathscr{F})$ corresponding to $A$ (resp. $B$ ), the induction hypothesis applied to $\bar{A}$ and $\bar{B}$ leads to the result that $p=m, r_{i}=s_{i}$, and $\left(D_{r_{1}}, \ldots, D_{r_{m}}\right)$ is conjugate to $\left(E_{r_{1}}, \ldots, E_{r_{m}}\right)$ in $H$.

We may thus assume $G$ is reductive. A simple argument allows us to work with semisimple $G$.

Let $G$ be semisimple. If $X=\left(A_{r}\right)_{s}=\left(B_{r}\right)_{s} \neq 0$, the reduction to $\mathrm{g}_{X}(\mathcal{F})$ under $G(\vartheta)_{1}$ guaranteed by Corollary 9.3.2, together with the facts $\operatorname{dim}\left(\mathrm{g}_{X}\right)<\operatorname{dim}(\mathrm{g}), N\left(\mathrm{~g}_{X}\right) \leq N(\mathrm{~g})$, proves what we want. So we may assume that $A_{r}=B_{r} \neq 0$ is nilpotent. As usual we take $A_{r}=Y$ and assume (\$9.3) that $A_{r+m}, B_{r+m} \in \mathrm{g}_{X}$ for all $m \geq 1$. We now argue as in Proposition 5.3 using Proposition 9.4. The key step is the verification that if $A^{\prime}=x[A], B^{\prime}=x[B]$ where $x=z^{-\delta H / 2}$, and if $\tilde{A}^{\prime}, \tilde{B}^{\prime}$ are the $\zeta$-liftings of $A^{\prime}$ and $B^{\prime}$ where $\zeta=z^{1 / 2 b}$, then $\tilde{A}_{\tilde{r}+s}^{\prime}=\tilde{B}_{\tilde{r}+s}^{\prime}, 0 \leq s<N(g)(|\tilde{r}|-1)$; 
here $\tilde{r}=2 b(r+\delta)+2 b-1=2 b r+2 a+2 b-1$. We know (cf. Corollary 3 to Proposition 4.6) that $\tilde{A}^{\prime} \equiv \tilde{B}^{\prime}(2 b M-2 a \Lambda)$ where $M=$ $N(\mathfrak{g})(|r|-1)$. So we must check that $N(\mathfrak{g})(|\tilde{r}|-1) \leq 2 b M-2 a \Lambda$. This is done exactly as in $\S 5.3$ using the estimate $\Lambda \leq N(\mathfrak{g})$ (Proposition 2.1.2). In fact, since $|\tilde{r}|-1=2 b(|r|-1-\delta)$ and $\Lambda \leq N(\mathfrak{g})$,

$$
2 b M-2 a \Lambda-N(\mathrm{~g})(|\tilde{r}|-1) \geq 2 b M-2 a N(\mathfrak{g})-N(\mathfrak{g})(|\tilde{r}|-1)=0 \text {. }
$$

9.8. In the remainder of this section we shall give a brief treatment of the theory of $G(\mathscr{F})$-equivalence of connections in $\mathfrak{g}(\mathscr{F})$.

DEFINITION. A canonical connection $B$ as in (9.1) is said to be weakly $b$-reduced if $r_{j} \in(1 / b) \mathbf{Z}$ for all $j$ and $C$ is weakly $b$-reduced as an element of $\mathfrak{g}_{D_{r_{1}}, \ldots, D_{r_{m}}}=$ centralizer of $D_{r_{1}}, \ldots, D_{r_{m}}$ in $\mathfrak{g}$. It is said to be weakly $\mathbf{Z}$-reduced if $C$ is a weakly $\mathbf{Z}$-reduced element of $\mathfrak{g}_{D_{r_{1}}, \ldots, D_{r_{m}}}$.

LEMMA 1. Let $B$ be any element of $g(\overline{\mathscr{F}})$. Then $B$ is $G(\overline{\mathscr{F}})$-equivalent to a canonical weakly $\mathbf{Z}$-reduced connection in $\mathrm{g}(\overline{\mathscr{F}})$.

Proof. We may assume that $B$ is itself canonical, say $B=D_{r_{1}} z^{r_{1}}$ $+\cdots+D_{r_{m}} z^{r_{m}}+z^{-1} C$. Let $z=g_{D_{r_{1}} \ldots, D_{r_{m}}}$; then $z=\operatorname{Lie}(Z)$ where $Z$ is the centralizer of $D_{r_{1}}, \ldots, D_{r_{m}}$ in $G$. We know that $Z$ is connected. Regarding $z^{-1} C$ as an element of $z(\mathscr{F})$ we can use Proposition 8.6 to reduce it to the form $z^{-1} C^{\prime}$ over $Z(\overline{\mathscr{F}}), C^{\prime}$ being a weakly Z-reduced element of $z$.

LEMMA 2. Let $B, B^{\prime}$ be canonical weakly b-reduced elements of $\mathfrak{g}\left(\mathscr{F}_{b}\right)$ with the same coefficient of $z^{-1}$. Then the set of all $x \in G\left(\mathscr{F}_{b}\right)$ such that $x[B]=B^{\prime}$ is contained in $G$ and coincides with the set of all $t \in G$ centralizing $C$ and taking $B_{\mathrm{irr}}$ to $B_{\mathrm{irr}}^{\prime}$.

Proof. Write $B^{\prime}=D_{r_{1}}^{\prime} z^{r_{1}}+\cdots+D_{r_{m}}^{\prime} z^{r_{m}}+z^{-1} C$ where we use the same determinations of the $z^{r_{J}}$ for both $B$ and $B^{\prime}$. By (9.2) we have, for any $x \in G(\overline{\mathscr{F}})$ such that $x[B]=B^{\prime}$, the relations $\operatorname{Ad}(x)\left(D_{r_{j}}\right)=D_{r_{j}}^{\prime}, 1 \leq j \leq m$, and $x\left[z^{-1} C\right]=z^{-1} C$. Lemma 8.7.1 implies now that $x \in G$ and centralizes $C$.

Proposition. Let $B=D_{r_{1}} z^{r_{1}}+\cdots+D_{r_{m}} z^{r_{m}}+z^{-1} C$ be weakly Z-reduced and canonical. Then $B$ is $G(\overline{\mathscr{F}})$-equivalent to an element of $\mathfrak{g}(\mathscr{F})$ if and only if there is an integer $b \geq 1$ and $a \theta \in G$ such that $\theta^{b}=1$, $\operatorname{Ad}(\theta)(C)=C, b r_{j}=s_{j} \in \mathbf{Z}, \operatorname{Ad}(\theta)\left(D_{r_{j}}\right)=\omega_{b}^{s} D_{r_{j}}$ for $1 \leq j \leq m$ where $\omega_{b}$ $=e^{2 \pi i / b}$. 
Proof. The "if" part is argued as in \$7.4. For the "only if" part let $d \geq 1$ be an integer such that $B \in \mathfrak{g}\left(\mathscr{F}_{d}\right)$ and $y^{-1}[B] \in \mathfrak{g}(\mathscr{F})$ for some $y \in G\left(\mathscr{F}_{d}\right)$. Applying Lemma 2 to $x=y\left(e^{2 \pi i / d} z^{1 / d}\right) y\left(z^{1 / d}\right)^{-1}$ we see that $x \in G, \operatorname{Ad}(x) C=C, \operatorname{Ad}(x)\left(D_{r_{j}}\right)=\omega_{d}^{t_{j}} D_{r_{j}}$ where $t_{j}=d r_{j} \in \mathbf{Z}$ and $\omega_{d}=$ $e^{2 \pi i / d}$. We may assume $x$ is semisimple. However $x^{d}$ need not be 1 . So we write $x=\exp (2 \pi i H)$ where $H \in \mathfrak{g}$ is semisimple and use Lemma 8.6 to select $R \in \mathcal{R}(H)$ such that $H+R$ is weakly $\mathbf{Z}$-reduced. Let $b \geq 1$ be divisible by $d$ and such that $\exp (2 \pi i b R)=1$ and let $t=\exp (2 \pi i(H+R))$. Then $t$ is semisimple, commutes with $x$, and $t^{b}=x^{b}$. Moreover, since $H+R$ is weakly $\mathbf{Z}$-reduced, $t$ and $t^{b}$ have the same centralizer in $\mathrm{g}$. Hence $t$ centralizes $C$ and the $D_{r_{j}}$. We then have $\theta=t^{-1} x$ with the required properties.

COROllary. $B$ is $G(\overline{\mathscr{F}})$-equivalent to an element of $g(\mathscr{F})$ if and only if the $G(\overline{\mathscr{F}})$-equivalence class of $B$ is $\mathrm{Gal}(\overline{\mathscr{F}} / \mathscr{F})$-invariant.

Proof. If there is Galois invariance we can find $d \geq 1$ and $x \in G\left(\mathscr{F}_{d}\right)$ such that $B \in \mathfrak{g}\left(\mathscr{F}_{d}\right)$ and $x[B]=B^{\prime}=D_{r_{1}} \omega_{d}^{t} z^{r_{1}}+\cdots+z^{-1} C$. The argument from this point on is the same as in the proposition.

Let $b \geq 1$ be an integer and

$$
B=D_{r_{1}} z^{r_{1}}+\cdots+D_{r_{m}} z^{r_{m}}+z^{-1} C, \quad b r_{j}=s_{j} \in \mathbf{Z},
$$

a weakly $b$-reduced canonical element of $\mathrm{g}\left(\mathscr{F}_{b}\right)$. As before let $\omega_{b}=e^{2 \pi i / b}$ and let $\Theta(b: B)$ be the set of all $\theta \in G$ such that

$$
\theta^{b}=1, \quad \operatorname{Ad}(\theta) C=C, \quad \operatorname{Ad}(\theta) D_{r_{j}}=\omega_{b}^{s_{j}} D_{r_{j}}, \quad 1 \leq j \leq m
$$

and let $T(b: B)$ be the set of all $t \in G$ such that

$$
\operatorname{Ad}(t) C=C, \quad \operatorname{Ad}(t) D_{r_{j}}=D_{r_{j}}, \quad 1 \leq j \leq m .
$$

It is obvious that $T(b: B)$ operates on $\Theta(b: B)$ by conjugation. For any $\theta \in \Theta(b: B)$ we write $[\theta]$ for the $T(b: B)$ orbit generated by $\theta$. We then have the following theorem. Its proof is along the same lines as in $\$ 7.4$ and is omitted.

TheOREM. Let $\Omega(b: B)$ be the set of all connections in $\mathfrak{g}(\mathcal{F})$ which are $G\left(\mathscr{F}_{b}\right)$-equivalent to $B$. For any $A \in \Omega(b: B)$ let $x \in G\left(\mathscr{F}_{b}\right)$ be such that $x[A]=B$. Then $\theta=x\left(\omega_{b} z^{1 / b}\right) x\left(z^{1 / b}\right)^{-1}$ lies in $\Theta(b: B)$ and $[\theta]$ is independent of the choice of $x$. Moreover, $[\theta]$ depends only on the $G(\mathcal{F})$-equivalence class of $A$, denoted by $[A]$; and the map $[A] \mapsto[\theta]$ gives a bijection

$$
G(\mathscr{F}) \backslash \Omega(b: B) \stackrel{\sim}{\rightarrow} T(b: B) \backslash \Theta(b: B) .
$$




\section{REFERENCES}

1. D. Bertrand, Travaux récents sur les points singuliers des équations différentielles linéaires, Sém. Bourbaki, 1978/79, Exposés 525-542, Lecture Notes in Mathematics No. 770, Berlin, Heidelberg, 1980.

2. G. D. Birkhoff, Equivalent singular points of ordinary linear differential equations, Math. Ann., 74 (1913), 134-139, Collected Mathematical Papers, Vol. 1, pp. 252-257, Dover Publications, New York, 1968.

3. A. Borel, Linear Algebraic Groups, W. A. Benjamin, New York, 1969.

4. N. Bourbaki, Éléments des Mathématiques, Livre II, Algébra Ch. 6-7 (2nd Ed.), Hermann, Paris.

5. E. Coddington, and N. Levinson, Theory of Ordinary Differential Equations, McGrawHill, New York, 1955.

6. F. Cope, Formal solutions of irregular linear differential equations, Amer. J. Math., 58 (1936), 130-140.

7. P. Deligne, Equations Différentielles à Points Singuliers Réguliers, Lecture Notes in Mathematics, No. 163, Springer-Verlag, Berlin, Heidelberg, 1970.

8. F. R. Gantmacher, Theory of Matrices, Vol. I, Chelsea, New York, 1959.

9. L_ Theory of Matrices, Vol. II, Chelsea, New York, 1959-60.

10. Harish-Chandra, Invariant distributions on Lie algebras, Amer. J. Math., 86 (1964), 271-309.

11. W. Jurkat, Meromorphe Differentialgleichungen, Lecture Notes in Mathematics, No. 637, Springer-Verlag, Berlin, Heidelberg, 1978.

12. W. Jurkat, and D. Lutz, On the order of solutions of analytic linear differential equations, Proc. London Math. Soc., 3 (1971), 465-482.

13. N. M. Katz, Nilpotent connections and the monodromy theorem: Applications of a result of Turrittin, Publ. Math. IHES, No. 39 (1970), 175-232.

14. B. Kostant, The principal three-dimensional subgroup and the Betti numbers of a complex simple Lie group, Amer. J. Math., 81 (1959), 973-1032.

$15 . \quad$ Lie group representations on polynomial rings, Amer. J. Math., 85 (1963), $327-404$.

16. A. Levelt, Jordan decomposition for a class of singular differential operators, Arkiv for Math., 13 (1975), 1-27.

17. D. Lutz, Asymptotic behavior of solutions of linear systems of ordinary differential equations near an irregular singular point, Amer. J. Math., 91 (1969), 95-105.

18. Yu Manin, Moduli fuchsiani, Ann. Sc. Norm. Pisa, III, 19 (1965), 113-126.

19. J. Moser, The order of a singularity in Fuchs' theory, Math. Z., 72 (1960), 379-398.

20. H. Poincaré, Sur les intégrals irregulières des équations linéaires, Acta Math., 8 (1886), 295-344, Oeuvres de Henri Poincaré, Tome 1, pp. 290-332, Gauthier-Villars, Paris, 1928.

21. L. Pukanszky, Lecons sur les représentations des Groupes, Dunod, Paris, 1967.

21a. P. Robba, Lemmes de Hensel pour les operateurs différentiels. Application a la reduction formelle des equations différentielles, L'Enseignement Mathematique, Ser. II, 26 (1980), 279-311.

22. J. P. Serre, Corps Locaux (2nd Ed.), Hermann, Paris, 1968.

23. __ Représentations Linéaires des Groupes Finis (2nd Ed.), Hermann, Paris, 1971.

24. T. A. Springer, and R. Steinberg, Conjugacy Classes in Lecture Notes in Mathematics, No. 131, Springer-Verlag Berlin, Heidelberg, 1970.

25. R. Steinberg, Lectures on Chevalley Groups, Dept. of Mathematics, Yale University, 1967.

26. H. Turrittin, Convergent solutions of ordinary linear homogeneous equations in the neighborhood of an irregular singular point, Acta Math., 93 (1955), 27-66. 
27. Reduction of ordinary differential equations to the Birkhoff canonical form, Trans. Amer. Math. Soc., 107 (1963), 485-507.

28. V. S. Varadarajan, On the ring of invariant polynomials on a semi-simple Lie algebra, Amer. J. Math., 90 (1968), 309-317.

29. , Harmonic Analysis on Real Reductive Groups, Lecture Notes in Mathematics, No. 576, Springer-Verlag, Berlin, Heidelberg, 1977.

30. _ Lie Groups, Lie Algebras, and Their Representations, Prentice-Hall, Englewood Cliffs, New Jersey, 1974.

31. W. Wasow, Asymptotic Expansions for Ordinary Differential Equations, Interscience, New York, 1965.

Received January 7, 1982 and in revised form April 28, 1982. Research of the first author was partially supported by NSF Grant MCS 78-01476, CNRS II in Marseille, the Université de Dijon and the Université de Toulon. The second author's research partially supported by NSF Grant MCS 79-03184 and the Mathematics Institute of the State University of Utrecht, Utrecht, Netherlands.

UNIVERSITY OF CALIFORNIA

LOS ANGELES, CA 90024 


\section{PACIFIC JOURNAL OF MATHEMATICS}

EDITORS

Donald BABBITt (Managing Editor)

University of California

Los Angeles, CA 90024

Hugo Rossi

University of Utah

Salt Lake City, UT 84112

C. C. Moore and Arthur Ogus

University of California

Berkeley, CA 94720

\section{J. DugundiI}

Department of Mathematics

University of Southern California

Los Angeles, CA 90089-1113

R. Finn and H. SAMELSON

Stanford University

Stanford, CA 94305

\section{ASSOCIATE EDITORS}
R. ARENS
E. F. BECKENBACH
B. H. NEUMANN
F. WOLF
K. YOSHIDA (1906-1982)

\section{SUPPORTING INSTITUTIONS}

UNIVERSITY OF ARIZONA

UNIVERSITY OF BRITISH COLUMBIA

CALIFORNIA INSTITUTE OF TECHNOLOGY

UNIVERSITY OF CALIFORNIA

MONTANA STATE UNIVERSITY

UNIVERSITY OF NEVADA. RENO

NEW MEXICO STATE UNIVERSITY

OREGON STATE UNIVERSITY
UNIVERSITY OF OREGION

UNIVERSITY OF SOUTHERN CALIFORNIA

STANFORD UNIVERSITY

UNIVERSITY OF HAWAII

UNIVERSITY OF TOKYO

UNIVERSITY OF UTAH

WASHINGTON STATE UNIVERSITY

UNIVERSITY OF WASHINGTON 


\section{Pacific Journal of Mathematics}

Vol. 109, No. $1 \quad$ May, 1983

Donald George Babbitt and V. S. Varadarajan, Formal reduction theory of meromorphic differential equations: a group theoretic view $\ldots \ldots \ldots \ldots 1$

Jo-Ann Deborah Cohen, Norms on $F(X) \ldots \ldots \ldots \ldots \ldots \ldots \ldots \ldots \ldots \ldots$

Robert Fitzgerald, Witt kernels of function field extensions $\ldots \ldots \ldots \ldots \ldots 89$

Hervé Jacquet and Joseph Andrew Shalika, The Whittaker models of induced representations .............................. 107

Masakiti Kinukawa, Some generalizations of contraction theorems for

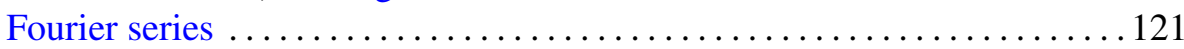

Joseph Weston Kitchen, Jr. and David A. Robbins, Sectional

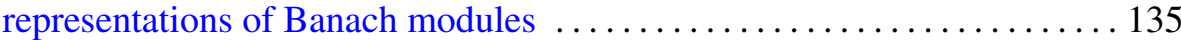

Victor Charles Pestien, Jr., Weak approximation of strategies in measurable gambling

Richard Scott Pierce and Charles Irvin Vinsonhaler, Realizing central

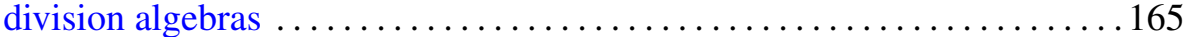

Walter Ricardo Ferrer Santos, Cohomology of comodules ............ 179

Marko Tadić, Harmonic analysis of spherical functions on reductive groups

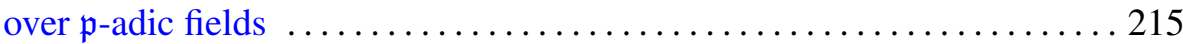

Lorenzo Traldi, The determinantal ideals of link modules. II . . . . . . . . 237

Alain J. Valette, A remark on the Kasparov groups $\operatorname{Ext}^{i}(A, B) \ldots \ldots \ldots 247$ 